Structure of attractors and estimates of their fractal dimension 

SERVIÇO DE PÓS-GRADUAÇÃO DO ICMC-USP

Data de Depósito:

Assinatura:

\title{
Structure of attractors and estimates of their fractal dimension
}

\author{
Matheus Cheque Bortolan
}

Advisor: Prof. Dr. Alexandre Nolasco de Carvalho

Doctoral dissertation submitted to the Instituto de Ciências Matemáticas e de Computação - ICMC-USP, in partial fulfillment of the requirements for the degree of the Doctorate Program in Computer Science and Computational Mathematics. FINAL VERSION.

USP - São Carlos

March 2013 
Ficha catalográfica elaborada pela Biblioteca Prof. Achille Bassi e Seção Técnica de Informática, ICMC/USP, com os dados fornecidos pelo(a) autor(a)

C739s $\begin{aligned} & \text { Cheque Bortolan, Matheus } \\ & \text { Structure of attractors and estimates of their } \\ & \text { fractal dimension / Matheus Cheque Bortolan; } \\ & \text { orientador Alexandre Nolasco de Carvalho. -- São } \\ & \text { Carlos, } 2013 \text {. } \\ & \text { 106 p. } \\ & \text { Tese (Doutorado - Programa de Pós-Graduação em } \\ & \text { Matemática) -- Instituto de Ciências Matemáticas e } \\ & \text { de Computação, Universidade de São Paulo, 2013. } \\ & \text { 1. fractal dimension. 2. skew product semiflow. } \\ & \text { 3. Morse decomposition. } 4 \text { gradient-like dynamical } \\ & \text { systems. 5. attractors of dynamical systems. I. } \\ & \text { Nolasco de Carvalho, Alexandre, orient. II. Título. }\end{aligned}$


SERVIÇO DE PÓS-GRADUAÇÃO DO ICMC-USP

Data de Depósito:

Assinatura:

\title{
Estrutura de atratores e estimativas de suas dimensões fractais
}

\author{
Matheus Cheque Bortolan
}

Orientador: Prof. Dr. Alexandre Nolasco de Carvalho

Tese apresentada ao Instituto de Ciências Matemáticas e de Computação - ICMC-USP, como parte dos requisitos para obtenção do título de Doutor em Ciências - Ciências de Computação e Matemática Computacional. VERSÃO REVISADA 
Ficha catalográfica elaborada pela Biblioteca Prof. Achille Bassi e Seção Técnica de Informática, ICMC/USP, com os dados fornecidos pelo(a) autor(a)

Cheque Bortolan, Matheus

C739e Estrutura de atratores e estimativas de suas dimensões fractais / Matheus Cheque Bortolan; orientador Alexandre Nolasco de Carvalho. -- São Carlos, 2013.

$106 \mathrm{p}$.

Tese (Doutorado - Programa de Pós-Graduação em Ciências de Computação e Matemática Computacional) -Instituto de Ciências Matemáticas e de Computação, Universidade de São Paulo, 2013.

1. dimensão fractal. 2. skew product semiflow. 3. decomposição de Morse. 4. sistemas dinâmicos gradientlike. 5. atratores de sistêmas dinâmicos. I. Nolasco de Carvalho, Alexandre, orient. II. Título. 
"Out of the night that covers me, Black as the pit from pole to pole, I thank whatever gods may be For my unconquerable soul.

In the fell clutch of circumstance I have not winced nor cried aloud. Under the bludgeonings of chance My head is bloody, but unbowed.

Beyond this place of wrath and tears Looms but the Horror of the shade, And yet the menace of the years Finds and shall find me unafraid.

It matters not how strait the gate, How charged with punishments the scroll. I am the master of my fate; I am the captain of my soul."

Invictus - William Ernest Henley 


\section{Acknowledgements}

Gostaria primeiramente de agradecer a Deus e à minha família. Todos eles estiveram ao meu lado em todos os momentos, me dando força e coragem quando eu mais precisei. Meus pais Vivaldo e Jandira, meus irmãos Marcelo e Márcia, meus cunhados Adaílton e Sheila, minhas sobrinhas Paola e Gretha, meus tios e tias Jorgina, Janet, Jayme e Judith, também a minha tia Jaymencita, que já não se encontra entre nós, e também todos os meus primos e primas... Sozinho eu não tenho um décimo da força que tenho quanto estou com eles ao meu lado. Amo todos vocês!

Agradeço ao meu orientador Prof. Alexandre e também ao prof. Hildebrando, que me ajudaram por todos esses anos, desde o primeiro ano da minha graduação... Agradeço também aos professores Tomás Caraballo e José Antonio Langa, que são pessoas excepcionais, me auxiliaram e apoiaram desde o primeiro dia que cheguei em Sevilha. Agradeço também a todos os professores que estiveram presentes durante a minha formação, desde a minha graduação até hoje; todos são modelos de pessoas e profissionais que eu vou ter para o resto da minha vida. Nada disso teria acontecido sem vocês, muito obrigado! Todos vocês tem meu eterno respeito e admiração!

Agradeço a todos os funcionários que me ajudaram por todos esses anos, em especial os funcionários das seções de graduação e pós-graduação, Ana Oneide Martins de Araujo Sáles, Ana Carolina Venere Murata, Ana Paula Sampaio Fregona, Glaucia Blangis, Laura Aparecida Donizeti Ruy Turi e Lhais Visentin, que sempre me aturaram fazendo milhares de perguntas, e me ajudaram durante todo este processo. Agradeço também a tantos outros que me ajudaram quando eu precisei, Marília, Fernanda Magro, Luana, Dóra, Diego, Michel e muitos outros... Muito obrigado a cada um de vocês!

Agradeço todos os amigos que fiz nessa jornada, Alex, Rodolfo, Giuliano, Mineiro, Gabriel, Moreno, Badaró, Thiago, Jorge, Rodrigo, Eduard, Northon, Nelson, Paulo, Bruno, Edinho, Rafael Borro, Rafael Rossato, Alexsandro, Felipe, Camila Ruiz, Camila Antunes, Thaís Maria, Thaís Jordão, Nazira, Jaqueline Ferreira, Carol Ramos, Carol Garcia, Maria Fernanda Mar- 
reta, Livia Rodrigues, Noemi, Jaqueline Mesquita, Luís Mesquita, Renato Alejandro, Rodrigo Cohen, Jéssyca e tantos outros... Não vou conseguir listar todos aqui porque senão precisaria de pelo menos umas 40 páginas, mas cada um de vocês sabe a importância que teve ne minha vida, e só tenho a agradecer por ter encontrado cada um de vocês! Agradeço às horas (infinitas) de estudo, às horas de lazer, às conversas, churrascos, jogos de vôlei e tudo o mais... Muito obrigado a todos vocês, de coração! Vocês sempre podem contar comigo pro que der e vier!

Agradeço à FAPESP, ao CNPq e à CAPES pelo apoio financeiro à este trabalho, sem este apoio nada disso seria possível.

Um homem nada mais é do que as ações que pratica, das pessoas que ama e dos amigos que tem. 


\section{Abstract}

This work is dedicated to the study of the structure of attractors of dynamical systems with the objective of estimating their fractal dimension. First we study the case of exponential global attractors of some generalized gradient-like semigroups in a general Banach space, and estimate their fractal dimension in terms of the maximum of the dimension of the local unstable manifolds of the isolated invariant sets, Lipschitz properties of the semigroup and rate of exponential attraction. We also generalize this result for some special evolution processes, introducing a concept of Morse decomposition with pullback attractivity. Under suitable assumptions, if $\left(A, A^{*}\right)$ is an attractor-repeller pair for the attractor $\mathscr{A}$ of a semigroup $\{T(t): t \geq 0\}$, then the fractal dimension of $\mathscr{A}$ can be estimated in terms of the fractal dimension of the local unstable manifold of $A^{*}$, the fractal dimension of $A$, the Lipschitz properties of the semigroup and the rate of the exponential attraction. The ingredients of the proof are the notion of generalized gradient-like semigroups and their regular attractors, Morse decomposition and a fine analysis of the structure of the attractors. Also, making use of the skew product semiflow and its Morse decomposition, we give some estimates of the fractal dimension of the pullback attractors of non-autonomous dynamical systems. 


\section{Resumo}

Este trabalho é dedicado ao estudo da estrutura dos atratores de sistemas dinâmicos com o objetivo de obter estimativas de suas dimensões fractais. Primeiramente estudamos o caso de atratores globais exponenciais de alguns semigrupos gradient-like generalizados em um espaço de Banach geral, e estimamos suas dimensões fractais em termos da máxima $d i$ mensão das variedades instáveis locais dos conjuntos invariantes isolados, a propriedades de Lipschitz do semigrupo e da taxa de atração exponencial. Também generalizamos este resultado para alguns processos de evoluções especiais, introduzindo um conceito de decomposição de Morse com atração pullback. Sob hipóteses apropriadas, se $\left(A, A^{*}\right)$ é um par atrator-repulsor para o atrator $\mathscr{A}$ de um semigrupo $\{T(t): t \geq 0\}$, então a dimensão fractal de $\mathscr{A}$ pode ser estimada em termos da dimensão fractal da variedade instável de $A^{*}$, a dimensão fractal de $A$, as propriedades de Lipschitz do semigrupo e a taxa de atração exponencial. Os ingredientes da demonstração são a noção de semigrupos gradient-like e seus atratores regulares, decomposição de Morse e uma análise fina da estrutura dos atratores. Além disto, fazendo uso do skew product semiflow e sua decomposição de Morse, damos estimativas da dimensão fractal dos atratores pullback de sistêmas dinâmicos não-autônomos. 


\section{Contents}

Introduction 1

1 Semigroups 5

1.1 Attractors for semigroups $\ldots \ldots \ldots \ldots \ldots \ldots \ldots$

1.1.1 Upper and lower semicontinuity of attractors . . . . . . . . . . . . . 10

1.2 Gradient semigroups . . . . . . . . . . . . . . . . . . . . . . 12

1.3 Generalized gradient-like semigroups . . . . . . . . . . . . . . . . . 13

1.4 Gradient-like semigroups are gradient semigroups . . . . . . . . . . . . . . 18

1.4.1 Morse decomposition of gradient-like semigroups . . . . . . . . . 18

1.4.2 Lyapunov functions and stability for gradient semigroups . . . . . . . . . 23

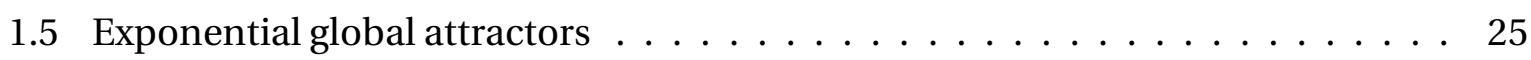

1.5.1 Gradient-like semigroups with exponential global attractors . . . . . . 25

2 Evolution processes $\quad 31$

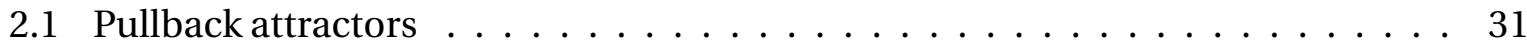

2.2 Existence of pullback attractors . . . . . . . . . . . . . . . . 34

2.3 Gradient-like evolution processes $\ldots \ldots \ldots \ldots \ldots \ldots \ldots$. . . . . . . . . 35

2.3.1 Definition and main properties . . . . . . . . . . . . . 35

2.3.2 Gradient-like processes under perturbation . . . . . . . . . . . . . 37

3 An estimate on the fractal dimension 39

3.1 Estimates on the fractal dimension $\ldots \ldots \ldots \ldots \ldots \ldots \ldots \ldots \ldots$

3.2 Non-autonomous dynamical systems and attractor-repeller pairs . . . . . . . 49

3.3 Morse decomposition for non-autonomous dynamical systems . . . . . . . . 59

3.4 An estimate on the fractal dimension of pullback attractors . . . . . . . . . . 63

3.5 Example . . . . . . . . . . . . . . . . . . . . . . 69 
4 Skew product semiflows and Morse decompositions $\quad 73$

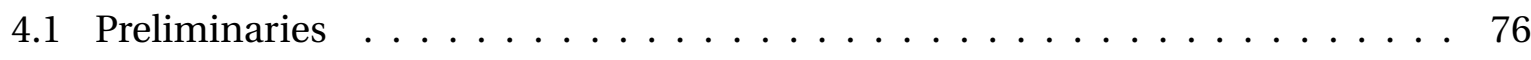

4.1.1 Non-autonomous dynamical systems and pullback attractors . . . . . 76

4.1.2 Time dependence and translations . . . . . . . . . . . . . . 79

4.1.3 Homoclinic structures in $\omega$-limit sets . . . . . . . . . . . . . . . 81

4.2 Morse decomposition for a skew product semiflow . . . . . . . . . . . . 82

4.2.1 The lift of a Morse decomposition from $P$ to $X \times P \ldots \ldots$. . . . . 82

4.2.2 The projection of a Morse decomposition from $X \times P$ to $P$. . . . . . . 84

4.3 Morse decomposition for pullback attractors . . . . . . . . . . . . . . . . 86

4.4 Small non-autonomous perturbations $\ldots \ldots \ldots \ldots \ldots$. . . . . . . . . 90

4.4.1 Asvmptotically autonomous evolution processes . . . . . . . . . . . . 92

4.5 Applications . . . . . . . . . . . . . . . . . . . . . 97 


\section{Introduction}

Over the last forty years, the study of qualitative properties of semigroups in Banach spaces has received very much attention (see for instance [2], [15], [17], [25] and [46]). In particular, the study of global attractors has created a deep area of research and greatly improved the understanding of qualitative properties of solutions for these infinite dimensional dynamical systems.

A particular aspect that has called the attention of many researchers, and for which a very nice theory has been developed, is the fractal dimension of attractors. Starting with the pioneering works [30] and [31], the theory has grown considerably and new strategies to find bounds for the fractal dimension have been proposed (see for example [46, 18, 25, 15] and references therein).

If we consider a compact set $K$ in a metric space $\left(X, d_{X}\right)$ we have the notions of topological dimension $\left(\operatorname{dim}_{T}(K)\right)$, Hausdorff dimension $\left(\operatorname{dim}_{H}(K)\right)$ and of the fractal dimension $(c(K))$ of $K$ (we will define all these notions in Chapter 3), and we know that $\operatorname{dim}_{T}(K) \leqslant$ $\operatorname{dim}_{H}(K) \leqslant c(K)$. Then one might ask why to study the fractal dimension, since it is the worst estimate for the dimensions of the same compact set $K$, and one particular result that makes the fractal dimension a very interesting object of research is the following result (see [31]):

Theorem 0.0.1. Given a Banach space $X$, a compact subset $K$ of $X$ with fractal dimension $c(K)<\infty$ and a finite dimensional subspace $Y$ with $\operatorname{dim} Y>2 c(K)+1$, if $\mathscr{P}(X, Y)$ is the subspace of $\mathscr{L}(X, Y)$ of the projections with range $Y$, the set $\left\{P \in \mathscr{P}(X, Y):\left.P\right|_{K}\right.$ is injective $\}$ is of second category in $\mathscr{P}(X, Y)$.

The inverse of the projection restricted to $K$ is continuous. In fact, in some situations, this inverse is Hölder continuous (see [20,41]). Another aspect is that the fractal dimension computation is fairly easy when we compare it with the computation of the Hausdorff and 
topological dimensions.

If a gradient semigroup $\{T(n): n \in \mathbb{N}\}$ has a global attractor $\mathscr{A}$ and its set of stationary solutions $\mathscr{E}$ is finite, then

$$
\mathscr{A}=\bigcup_{e \in \mathscr{E}} W^{u}(e)
$$

where we recall that

$W^{u}(e)=\{x \in X:$ there is a global solution $\phi: \mathbb{Z} \rightarrow X$ through $x$ such that $\phi(n) \stackrel{n \rightarrow-\infty}{\longrightarrow} e\}$.

For gradient semigroups we know that the local unstable set $W_{\mathrm{loc}}^{u}(e)$ is the intersection of $W^{u}(e)$ with a neighborhood of $e$. Assume that $W_{\text {loc }}^{u}(e)$ is the graph of a Lipschitz map with domain in a finite dimensional afine linear manifold $e+Q_{e}(X)$ where $Q_{e}$ is a projection with finite dimensional rank.

We know (following [12]) that, using the results on the Hausdorff dimension $\operatorname{dim}_{H}(\cdot)$, we have

$$
\begin{aligned}
& \operatorname{dim}_{H}\left(W_{\text {loc }}^{u}(e)\right)=\operatorname{rank}\left(Q_{e}\right)<\infty, \text { for each } e \in \mathscr{E}, \\
& \operatorname{dim}_{H}\left(T(n) W_{\text {loc }}^{u}(e)\right) \leqslant \operatorname{dim}_{H}\left(W_{\operatorname{loc}}^{u}(e)\right), n \geq 0
\end{aligned}
$$

It is not difficult to see that $W^{u}(e)=\cup_{n=0}^{\infty} T(n) W_{\text {loc }}^{u}(e)$ and, from the $\sigma$-sub-additivity property of the Hausdorff measure it follows that

$$
\begin{aligned}
\operatorname{rank}\left(Q_{e}\right) & =\operatorname{dim}_{H}\left(W_{\mathrm{loc}}^{u}(e)\right) \\
& \leqslant \operatorname{dim}_{H}\left(W^{u}(e)\right) \\
& =\operatorname{dim}_{H}\left(\bigcup_{n=0}^{\infty} T(n) W_{\mathrm{loc}}^{u}(e)\right) \\
& \leqslant \sup _{n \in \mathbb{N}} \operatorname{dim}_{H}\left(T(n) W_{\mathrm{loc}}^{u}(e)\right) \\
& \leqslant \operatorname{dim}_{H}\left(W_{\mathrm{loc}}^{u}(e)\right) \\
& =\operatorname{rank}\left(Q_{e}\right),
\end{aligned}
$$

and therefore $\operatorname{dim}_{H}\left(W^{u}(e)\right)=\operatorname{rank}\left(Q_{e}\right)$, for all $e \in \mathscr{E}$. Hence, since $\mathscr{A}=\bigcup_{e \in \mathscr{E}} W^{u}(e)$, we have that

$$
\operatorname{dim}_{H}(\mathscr{A})=\max _{e \in \mathscr{E}} \operatorname{rank}\left(Q_{e}\right)
$$

In particular $\mathscr{A}$ is homeomorphic to a subset of $\mathbb{R}^{N}$ where $N=2 \max _{e \in \mathscr{E}} \operatorname{rank}\left(Q_{e}\right)+1$.

It would be very nice to be able to prove a similar result to $\circledast$ for the fractal dimension, 
and this will be indeed one of our main objective in this work. Nonetheless, such result would not be expected since the manner in which the unstable manifold of a given equilibria accumulates on other equilibria may be at a slow rate causing the dimension to increase (like it happens with the set $\left\{\frac{1}{n}: n \in \mathbb{N}^{*}\right\} \cup\{0\}$ ). However, if we take the sequence $\left\{\frac{1}{2^{n}}: n \in \mathbb{N}\right\} \cup\{0\}$, it is not difficult to see that the Hausdorff and fractal dimension coincide. Inspired by this, we seek a bound for the fractal dimension of regular attractors with exponentially attracting local unstable manifolds.

The result will be proved for generalized gradient-like semigroups in Chapter 3 and will make use of the Morse decomposition of a generalized gradient-like semigroup. In Section 1.4.1 we introduce the basic concepts and results needed to prove the main result. Section 3.1 is dedicated to obtain an estimate on the fractal dimension of global attractors for generalized gradient-like Lipschitz semigroups for which the local unstable set of an isolated invariant set is the graph of a Lipschitz map over a finite dimensional subspace of the phase space.

In Chapters 1 and 2 we give a brief introduction to the theory of semigroups and evolution processes, respectively. Some of the results are quite recent, as the stability of gradient semigroups under perturbations, and can be found in [1].

In Chapter 4we will apply the estimates seen on Chapter 3 to non-autonomous dynamical systems, and to this end we will study the skew product semiflows and the behavior of a Morse decomposition for this object. More precisely in Section 4.1 we recall and state some of the basic definitions and set the notation we will use throughout the chapter.

In Section 4.2 we construct a Morse decomposition for the global attractor of the skew product semiflow $\{\Pi(t): t \geqslant 0\}$ given a Morse decomposition for the global attractor of the driving system $\{\Theta(t): t \geqslant 0\}$, using the lift of this Morse decomposition. We also verify under which conditions a given Morse decomposition in the global attractor of the skew product semiflow $\{\Pi(t): t \geqslant 0\}$ generates a Morse decomposition in the global attractor of the driving system $\{\Theta(t): t \geqslant 0\}$.

In Section 4.3 we construct a Morse decomposition for the pullback attractor of a nonautonomous dynamical system and we obtain some dynamical properties of this Morse decomposition, such as forward convergence, pullback convergence and the existence of a Lyapunov function.

In Section 4.4 we describe how a Morse Decomposition of a pullback attractor is stable under perturbation on the parameter of the associated driving system.

Finally, in Section 4.5]we present some applications of our theory, such as a non-autonomous 
differential equation defined only for $t \geqslant 0$

$$
\left\{\begin{array}{l}
\dot{x}=f(t, x), \quad t>0, \\
x(0)=x_{0} \in \mathbb{R}^{n}
\end{array}\right.
$$

an asymptotically autonomous (backward and forwards) non-autonomous differential equation, i.e.

$$
\left\{\begin{array}{l}
\dot{x}=f(t, x), \quad t \in \mathbb{R}, \\
x(0)=x_{0} \in \mathbb{R}^{n},
\end{array}\right.
$$

where

$$
\sup _{x \in \mathbb{R}^{n}}\left\|f(t, x)-f_{2}(x)\right\|_{\mathbb{R}^{n}} \stackrel{t \rightarrow \infty}{\longrightarrow} 0, \quad \text { and } \quad \sup _{x \in \mathbb{R}^{n}}\left\|f(t, x)-f_{1}(x)\right\|_{\mathbb{R}^{n}} \stackrel{t \rightarrow-\infty}{\longrightarrow} 0,
$$

for suitable functions $f_{1}$ and $f_{2}$, and also a master-slave example, i.e. a system of partially coupled equations

$$
\left\{\begin{array}{l}
\dot{v}=f(u, v) \quad t>0 \\
\dot{u}=g(u), \quad t>0 \\
u(0)=u_{0} \in \mathbb{R}^{n}, v(0)=v_{0} \in \mathbb{R}^{n}
\end{array}\right.
$$

in which the second equation for $u(t)$ acts as a driving system for the unknown $v(t)$. Finally we present a more concrete example to illustrate the use of the abstract theory, studying the behavior of an planar system of ODE's given by

$$
\frac{d}{d t}(x, y)=F(t,(x, y)), \quad t \in \mathbb{R}
$$

where $F: \mathbb{R} \times \mathbb{R}^{2} \rightarrow \mathbb{R}^{2}$ has some special properties.

It is worth to point out that Chapters 3 and 4 describe the results obtained during the development of this thesis. 


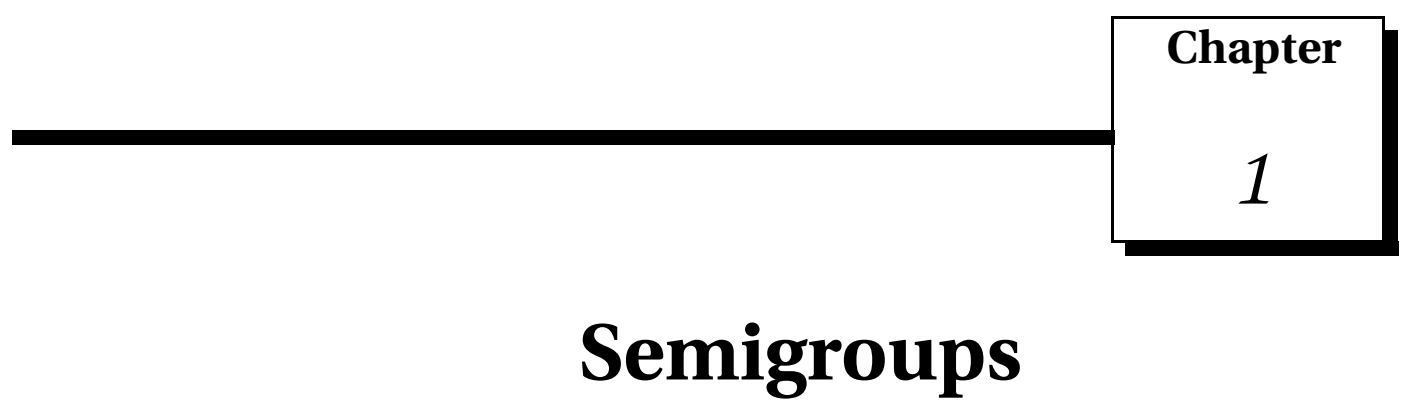

In this first chapter our goal is to introduce the concept of semigroups and its properties. This concept is very common in the literature, and it has been extensively studied by many authors (see [2, 15, 17, 18, 25, 36]). We can see in these references that the object that plays a fundamental role in the study of asymptotic dynamics for a semigroup is the global attractor, which we will see in the following section. Our purpose here (and also in Chapter 2) is to make a brief introduction to the theory of semigroups (evolutions processes in Chapter 2) and therefore we will not give the proof of all the results, only the ones that are essential for the purpose of this work.

\subsection{Attractors for semigroups}

In this section we introduce the basic concepts and results that lead us to the characterization of the semigroups which possess a global attractor.

Let $X$ be a metric space and $d: X \times X \rightarrow[0, \infty)$ its metric. Denote by $\mathscr{C}(X)$ the set of all continuous maps from $X$ into itself.

We will write $\mathbb{T}$ to denote either the set of the integers $\mathbb{Z}$ or the set of real numbers $\mathbb{R}$, $\mathbb{T}^{+}=\{t \in \mathbb{T}: t \geqslant 0\}, \mathbb{T}^{-}=\{t \in \mathbb{T}: t \leqslant 0\}, \mathbb{T}_{t}^{-}=t+\mathbb{T}^{-}$and $\mathbb{T}_{t}^{+}=t+\mathbb{T}^{+}$.

Given $K \subset X$ and $r>0$, the $r$-neighborhood of $K$ is the set defined by $\mathscr{O}_{r}(K):=\{x \in X$ : $d(x, K)<r\}$.

Definition 1.1.1. A semigroup is a family $\left\{T(t): t \in \mathbb{T}^{+}\right\} \subset \mathscr{C}(X)$ such that 
(i) $T(0) x=x$, for all $x \in X$,

(ii) $T(t+s)=T(t) T(s)$, for all $t, s \in \mathbb{T}^{+}$,

(iii) $\mathbb{T}^{+} \times X \ni(t, x) \mapsto T(t) x \in X$ is continuous.

In the case that $\mathbb{T}=\mathbb{Z}$, the third condition is automatically satisfied and, since $T(n)=$ $T(1)^{n}$, taking $T:=T(1)$, the semigroup can be rewritten as $\left\{T^{n}: n \in \mathbb{N}\right\}$ and will be simply the family of operators $\left\{T^{n}: n \in \mathbb{N}\right\} \subset \mathscr{C}(X)$.

Given a semigroup $\left\{T(t): t \in \mathbb{T}^{+}\right\} \subset \mathscr{C}(X)$ and a subset $B$ of $X$, we define:

(a) For each $t \in \mathbb{T}^{+}$, the image of $B$ under $T(t)$ by

$$
T(t) B \doteq\{T(t) x: x \in B\} ;
$$

(b) The positive orbit of $B$ by

$$
\gamma^{+}(B) \doteq \bigcup_{t \in \mathbb{T}^{+}} T(t) B
$$

(c) The partial orbit between two numbers $t, t^{\prime} \in \mathbb{T}^{+}$with $t<t^{\prime}$ by

$$
\gamma_{\left[t, t^{\prime}\right]}^{+}(B) \doteq \bigcup_{s \in\left[t, t^{\prime}\right] \cap \mathbb{T}^{+}} T(s) B
$$

(d) The orbit of $T(t) B$ by

$$
\gamma_{t}^{+}(B) \doteq \bigcup_{s \in \mathbb{T}^{+}} T(s+t) B=\bigcup_{s \in \mathbb{T}_{t}^{+}} T(s) B
$$

For each $x \in X$, the function $\mathbb{T}^{+} \ni t \mapsto T(t) x \in X$ is the solution through $x$ of the semi$\operatorname{group}\left\{T(t): t \in \mathbb{T}^{+}\right\}$.

Definition 1.1.2. A semigroup $\left\{T(t): t \in \mathbb{T}^{+}\right\}$is said eventually bounded if for every bounded set $B \subset X$ there exists a $t_{B} \in \mathbb{T}^{+}$such that $\gamma_{t_{B}}^{+}(B)$ is bounded. We say that $\left\{T(t): t \in \mathbb{T}^{+}\right\}$is a bounded semigroup if $\gamma^{+}(B)$ is bounded for all bounded sets $B \subset X$.

Remark 1.1.3. The fact that $T \in \mathscr{C}(X)$ does not imply that $T$ takes bounded subsets of $X$ into bounded subsets of $X$, since bounded subsets of $X$ are not necessarily precompact. If we assume that $T$ is bounded in bounded subsets of $X$, considering the semigroup $\left\{T^{n}: n \in \mathbb{N}\right\}$, we have that $\gamma_{\left[n, n^{\prime}\right]}^{+}(B)$ is bounded for each bounded subset $B$ of $X$ and $n, n^{\prime} \in \mathbb{N}$. In the case 
that $\mathbb{T}^{+}=\mathbb{R}^{+}$, to obtain that $\{T(t): t \geqslant 0\}$ is bounded, we need to assume that $\{T(t): t \geqslant 0\}$ is eventually bounded and that $\gamma_{\left[0, T_{B}\right]}^{+}(B)$ is bounded for all $T_{B} \geqslant 0$ and for all $B \subset X$ bounded.

The set where the orbit of $B$ accumulates is called $\omega$-limit set and plays an important role in the study of the asymptotic behavior of a semigroup.

Definition 1.1.4. The $\omega$-limit set of a subset $B$ of $X$ is defined by

$$
\omega(B)=\bigcap_{t \in \mathbb{T}^{+}} \overline{\gamma_{t}^{+}(B)}
$$

Remark 1.1.5. Here, for a subset $K \subset X, \bar{K}$ denotes the closure of $K$ in $X$.

Definition 1.1.6. A global solution of $\left\{T(t): t \in \mathbb{T}^{+}\right\}$through $x \in X$ is a continuous function $\phi: \mathbb{T} \rightarrow X$ such that $\phi(0)=x$ and $T(t) \phi(s)=\phi(t+s)$, for all $t \in \mathbb{T}^{+}$and $s \in \mathbb{T}$. A constant global solution will be called stationary solution and its value is called an equilibrium point or a fixed point. Since $T(t)$ is not necessarily injective, if there exists a global solution, it does not need to be unique. When a global solution $\phi: \mathbb{T} \rightarrow X$ through $x \in X$ exists, we define the global orbit of $x$ relative to the global solution $\phi$ by $\gamma_{\phi}(x):=\{\phi(t): t \in \mathbb{T}\}$. In this case, for $t \in \mathbb{T}$ we write $\left(\gamma_{\phi}\right)_{t}^{-}(x):=\{\phi(s): s \leqslant t, s \in \mathbb{T}\}$ and define the $\alpha$-limit set of $x$ relative to $\phi$ by

$$
\alpha_{\phi}(x)=\bigcap_{t \in \mathbb{T}^{+}} \overline{\left(\gamma_{\phi}\right)_{t}^{-}(x)}
$$

The following characterization for the $\omega$-limit set will be frequently used in the upcoming results and its proof is very straightforward.

Proposition 1.1.7. If $B \subset X$, then $\omega(B)$ is closed and

$$
\begin{gathered}
\omega(B)=\left\{y \in X: \text { there exist sequences }\left\{t_{n}\right\}_{n \in \mathbb{N}} \text { in } \mathbb{T}^{+} \text {and }\left\{x_{n}\right\}_{n \in \mathbb{N}} \text { in } B\right. \\
\text { such that } \left.t_{n} \stackrel{n \rightarrow \infty}{\longrightarrow} \infty \text { and } y=\lim _{n \rightarrow \infty} T\left(t_{n}\right) x_{n}\right\}
\end{gathered}
$$

If $\phi: \mathbb{T} \rightarrow X$ is a global solution of the semigroup $\left\{T(t): t \in \mathbb{T}^{+}\right\}$through $x \in X$, then $\alpha_{\phi}(x)$ is closed and

$$
\alpha_{\phi}(x)=\left\{v \in X: \text { there exists a sequence }\left\{t_{n}\right\}_{n \in \mathbb{N}} \text { in } \mathbb{T}^{+} \text {such that } t_{n} \stackrel{n \rightarrow \infty}{\longrightarrow} \infty \text { and } \phi\left(-t_{n}\right) \rightarrow v\right\} .
$$


In what follows we define the notions of attraction, absorption and invariance under the action of the semigroup $\left\{T(t): t \in \mathbb{T}^{+}\right\}$. To this end, we remember the definition of the Hausdorff semidistance $\mathrm{d}_{H}(A, B)$ between two subsets $A$ and $B$ of $X$

$$
\mathrm{d}_{H}(A, B) \doteq \sup _{x \in A} \inf _{y \in B} d(x, y)
$$

We will denote by $\operatorname{dist}(A, B)$ the usual distance between sets; that is,

$$
\operatorname{dist}(A, B) \doteq \inf _{x \in A} \inf _{y \in B} d(x, y)
$$

Let $X$ be a metric space and $\left\{T(t): t \in \mathbb{T}^{+}\right\} \subset \mathscr{C}(X)$ be a semigroup.

Definition 1.1.8. If $A$ and $B$ are subsets of $X$, we say that $A$ attracts $B$ under the action of $\left\{T(t): t \in \mathbb{T}^{+}\right\}$if

$$
\lim _{t \rightarrow \infty} \mathrm{d}_{H}(T(t) B, A)=0
$$

If there exists $t_{0} \in \mathbb{T}^{+}$such that $T(t) B \subset A$ for all $t \geqslant t_{0}, t \in \mathbb{T}^{+}$, we say that $A$ absorbs $B$. In particular, if A absorbs B then A attracts B (the converse is not true).

The notion of invariance, given below, plays an important role on the study of the asymptotical dynamics of semigroups.

Definition 1.1.9. We say that a subset $A$ of $X$ is invariant (or positively invariant) under the action of $\left\{T(t): t \in \mathbb{T}^{+}\right\}$if $T(t) A=A($ or $T(t) A \subset A)$ for all $t \in \mathbb{T}^{+}$. An invariant unitary set corresponds to an equilibrium point of $\left\{T(t): t \in \mathbb{T}^{+}\right\}$; that is, a point $x^{*} \in X$ such that $T(t) x^{*}=x^{*}$ for all $t \in \mathbb{T}^{+}$.

Finally, we are in condition to define the global attractor for a semigroup.

Definition 1.1.10. A set $\mathscr{A}$ is called a global attractor for $\left\{T(t): t \in \mathbb{T}^{+}\right\}$if it is compact, invariant and attracts bounded subsets of $X$ under the action of $\left\{T(t): t \in \mathbb{T}^{+}\right\}$.

Note that the global attractor for a semigroup $\left\{T(t): t \in \mathbb{T}^{+}\right\}$is unique. Indeed, if $\mathscr{A}$ and $\hat{\mathcal{A}}$ are global attractors for this semigroup,

$$
\operatorname{dist}_{H}(\mathscr{A}, \hat{\mathscr{A}})=\operatorname{dist}_{H}(T(t) \mathscr{A}, \hat{\mathscr{A}}) \stackrel{t \rightarrow \infty}{\longrightarrow} 0
$$

and thus $\mathscr{A} \subset \hat{\mathscr{A}}$. Analogously $\hat{\mathscr{A}} \subset \mathscr{A}$ and we have the result. 
Remark 1.1.11. Let $\left\{T(t): t \in \mathbb{T}^{+}\right\}$be a semigroup in a metric space $X$. Assume that $\{T(t): t \in$ $\left.\mathbb{T}^{+}\right\}$has a global attractor $\mathscr{A}$. We claim that, given $x \in \mathscr{A}$, there exists a bounded global solution $\phi_{x}: \mathbb{T} \rightarrow X$ such that $\phi_{x}(0)=x$. Indeed, $\mathbb{T}^{+} \ni t \mapsto \phi(t):=T(t) x \in X$ is always well-defined, now let $x \in \mathscr{A}=T(1) \mathscr{A}$, hence there exists $x_{-1} \in \mathscr{A}$ such that $T(1) x_{-1}=x$ and proceeding inductively, we obtain a sequence $\left\{x_{-n}: n \in \mathbb{N}\right\}$ such that $x_{0}=x$ and $T(1) x_{-n-1}=x_{-n}$ for all $n \in \mathbb{N}$ (remember that the sequence $\left\{x_{-n}\right\}_{n \in \mathbb{N}}$ is not uniquely determined). Define

$$
\phi_{x}(t)=\left\{\begin{array}{l}
T(t) x, t \geqslant 0 \\
T(j+t) x_{-j}, t \in[-j,-j+1) \cap \mathbb{T}, j=1,2,3, \cdots
\end{array}\right.
$$

which is a bounded global solution in $\mathscr{A}$ through $x$ in $t=0$.

Conversely, each bounded global solution $\phi: \mathbb{R} \rightarrow X$ for $\left\{T(t): t \in \mathbb{T}^{+}\right\}$is such that $\phi(\mathbb{T}) \subset$ $\mathscr{A}$. Having this, we conclude that

$$
\mathscr{A}=\{x \in X: \text { there exists a bounded global solution through } x\}
$$

The next concepts are crucial in the characterization of the semigroups that possess a global attractor.

Definition 1.1.12. A semigroup $\left\{T(t): t \in \mathbb{T}^{+}\right\}$is said asymptotically compact if, for any nonempty, closed and bounded subset $B \subset X$, such that $T(t) B \subset B$, for all $t \in \mathbb{T}^{+}$, there exists a compact set $J \subset B$ which attracts $B$.

Definition 1.1.13. We say that a semigroup $\left\{T(t): t \in \mathbb{T}^{+}\right\}$is point dissipative (bounded dissipative/compact dissipative) if there exists a bounded subset $B \subset X$ which attracts points (bounded subsets/compact subsets) of X.

Remark 1.1.14. In the definition above, we can change the word attracts by the word absorbs without changing the meaning of the concepts.

The following theorem characterizes the semigroups which have global attractors.

Theorem 1.1.15. A semigroup $\left\{T(t): t \in \mathbb{T}^{+}\right\}$is eventually bounded, point dissipative and asymptotically compact if and only if $\left\{T(t): t \in \mathbb{T}^{+}\right\}$has a global attractor $\mathscr{A}$. 


\subsubsection{Upper and lower semicontinuity of attractors}

In this section we define the continuity of attractors relative to some perturbations of the semigroup.

Definition 1.1.16. Let $X$ be a metric space, $d(\cdot, \cdot): X \times X \rightarrow \mathbb{R}^{+}$its metric, $\Lambda$ a metric space and $\left\{\mathscr{A}_{\lambda}\right\}_{\lambda \in \Lambda}$ a family of subsets of $X$ and $\lambda_{0} \in \Lambda$.

(i) We say that $\left\{\mathscr{A}_{\lambda}\right\}_{\lambda \in \Lambda}$ is upper semicontinuous at $\lambda_{0}$ if

$$
\mathrm{d}_{H}\left(\mathscr{A}_{\lambda}, \mathscr{A}_{\lambda_{0}}\right)=\sup _{x_{\lambda} \in \mathscr{A}_{\lambda}} \operatorname{dist}\left(x_{\lambda}, \mathscr{A}_{\lambda_{0}}\right) \stackrel{\lambda \rightarrow \lambda_{0}}{\longrightarrow} 0
$$

(ii) We say that $\left\{\mathscr{A}_{\lambda}\right\}_{\lambda \in \Lambda}$ is lower semicontinuous at $\lambda_{0}$ if

$$
\mathrm{d}_{H}\left(\mathscr{A}_{\lambda_{0}}, \mathscr{A}_{\lambda}\right)=\sup _{x \in \mathscr{A}_{\lambda_{0}}} \operatorname{dist}\left(x, \mathscr{A}_{\lambda}\right) \stackrel{\lambda \rightarrow \lambda_{0}}{\longrightarrow} 0
$$

To prove the upper and lower semicontinuity we often use the following characterization result:

Lemma 1.1.17. Let $\Lambda$ a metric space and $\left\{\mathscr{A}_{\lambda}\right\}_{\lambda \in \Lambda}$ a family of subsets of $X$.

1. If any sequence $\left\{x_{\lambda_{n}}\right\}$ with $x_{\lambda_{n}} \in \mathscr{A}_{\lambda_{n}}, \lambda_{n} \stackrel{n \rightarrow \infty}{\longrightarrow} \lambda_{0}$, has a convergent subsequence with the limit belonging to $\mathscr{A}_{\lambda_{0}}$, then $\left\{\mathscr{A}_{\lambda}\right\}_{\lambda \in \Lambda}$ is upper semicontinuous at $\lambda_{0}$. Conversely, if $\left\{\mathscr{A}_{\lambda}\right\}_{\lambda \in \Lambda}$ is upper semicontinuous at $\lambda_{0}$, any sequence $\left\{x_{\lambda_{n}}\right\}$ with $x_{\lambda_{n}} \in \mathscr{A}_{\lambda_{n}}$ has a convergente subsequence with the limit belonging to $\mathscr{A}_{0}$.

2. If $\mathscr{A}_{\lambda_{0}}$ is compact and, for any $x \in \mathscr{A}_{\lambda_{0}}$ and $\lambda_{n} \stackrel{n \rightarrow \infty}{\longrightarrow} \lambda_{0}$, there exists a sequence $\lambda_{n_{k}} \stackrel{k \rightarrow \infty}{\longrightarrow}$ $\lambda_{0}$, and sequence $\left\{x_{\lambda_{n_{k}}}\right\}$ with $x_{\lambda_{n_{k}}} \in \mathscr{A}_{\lambda_{n_{k}}}$ which converges to $x$, then $\left\{\mathscr{A}_{\lambda}\right\}_{\lambda \in \Lambda}$ is lower semicontinuous at $\lambda_{0}$. Conversely, if $\left\{\mathscr{A}_{\lambda}\right\}_{\lambda \in \Lambda}$ is lower semicontinuous at $\lambda_{0}, \lambda_{n} \stackrel{n \rightarrow \infty}{\rightarrow} \lambda_{0}$ and $x \in \mathscr{A}_{0}$, there exist subsequence $\lambda_{n_{k}} \stackrel{k \rightarrow \infty}{\rightarrow} \lambda_{0}$ and sequence $\left\{x_{\lambda_{n_{k}}}\right\}$ with $x_{\lambda_{n_{k}}} \in \mathscr{A}_{\lambda_{n_{k}}}$ which converges to $x$.

Definition 1.1.18. We say that the family of semigroups $\left\{T_{\eta}(t): t \in \mathbb{T}^{+}\right\}, \eta \in[0,1]$, is continuous at $\eta=0$ if $T_{\eta}(t) x \stackrel{\eta \rightarrow 0}{\longrightarrow} T_{0}(t) x$ uniformly for $(t, x)$ in compact subsets of $\mathbb{1}^{+} \times X$. 
The upper semicontinuity of attractors is true in almost every case, as we can see with the next result.

Theorem 1.1.19 (Upper semicontinuity). $\operatorname{Let}\left\{T_{\eta}(t): t \in \mathbb{T}^{+}\right\}, \eta \in[0,1]$, be a continuous family of semigroups at $\eta=0$. If $\left\{T_{\eta}(t): t \in \mathbb{T}^{+}\right\}$has a global attractor $\mathscr{A}_{\eta}$ for each $\eta \in[0,1]$ and $\overline{\cup_{\eta \in[0,1]} \mathscr{A}_{\eta}}$ is compact, then the family $\left\{\mathscr{A}_{\eta}: \eta \in[0,1]\right\}$ is upper semicontinuous at $\eta=0$.

The lower semicontinuity is not as common as the upper semicontinuity, and it is also harder to verify in the applications. It requires a finer study of local structures (the local unstable sets of invariant sets), in particular of the local unstable sets of equilibria, as we will see below.

Given a semigroup $\left\{T(t): t \in \mathbb{T}^{+}\right\}$and $A$ an invariant bounded set under the action of $\left\{T(t): t \in \mathbb{T}^{+}\right\}$, we have already seen that there exists a bounded global solution through $a$, for each $a \in A$. The simplest global solutions are the constants; that is, the equilibrium points. The class of global solutions that converge to an equilibrium $y^{*}$ as $t$ goes to $-\infty$ form a set that we call unstable set $W^{u}\left(y^{*}\right)$ of $y^{*}$; that is,

$$
\begin{aligned}
& W^{u}\left(y^{*}\right)=\left\{y \in X: \text { there exists a global solution } \phi_{y}: \mathbb{T} \rightarrow X\right. \\
& \text { such that, } \left.\phi_{y}(0)=y \text { and } \phi_{y}(t) \stackrel{t \rightarrow-\infty}{\longrightarrow} y^{*}\right\} .
\end{aligned}
$$

Given a neighborhood $V$ of $y^{*}$, the set of points $y$ of $V$ such that there exists a global solution $\phi_{y}: \mathbb{T} \rightarrow X$ such that $\phi_{y}(0)=y, \phi_{y}(t) \stackrel{t \rightarrow-\infty}{\longrightarrow} y^{*}$ and $\phi_{y}(t) \in V$ for all $t \in \mathbb{T}^{-}$is called a local unstable set of $y^{*}$ and is denoted by $W_{\mathrm{loc}}^{u}\left(y^{*}\right)$.

With this object and some additional assumptions, we can obtain the lower semicontinuity of attractors.

Theorem 1.1.20 (Lower semicontinuity). Let $\left\{T_{\eta}(t): t \in \mathbb{T}^{+}\right\}, \eta \in[0,1]$, be a continuous family of semigroups at $\eta=0$ which satisfies

(a) $\left\{T_{\eta}(t): t \in \mathbb{T}^{+}\right\}$has a global attractor $\mathscr{A}_{\eta}$, for each $\eta \in[0,1]$.

(b) If $\mathscr{E}_{\eta}$ denotes the set of the stationary solutions of $\left\{T_{\eta}(t): t \in \mathbb{T}^{+}\right\}$, there exists a $\mathfrak{p} \in \mathbb{N}$ such that $\mathscr{E}_{\eta} \supset\left\{y_{1}^{*, \eta}, \cdots, y_{\mathfrak{p}}^{*, \eta}\right\}$, for all $\eta \in[0,1]$.

(c) If $W_{\delta}^{u}\left(y_{j}^{*, \eta}\right)=\left\{y \in \mathscr{O}_{\delta}\left(y_{j}^{*, \eta}\right)\right.$ : there exists a global solution $\phi_{y}: \mathbb{T} \rightarrow X$ such that, $\phi_{y}(0)=y$, $\phi_{y}(t) \stackrel{t \rightarrow-\infty}{\longrightarrow} y^{*}$ and $\phi_{y}(t) \in \mathscr{O}_{\delta}\left(y_{j}^{*, \eta}\right)$ for all $\left.t \in \mathbb{T}^{-}\right\}$. Assume that, for some $\delta>0$ suffi- 
ciently small,

$$
\left\{W_{\delta}^{u}\left(y_{j}^{*, \eta}\right): \eta \in[0,1]\right\}
$$

is lower semicontinuous at $\eta=0$, for each $j=1, \cdots, \mathfrak{p}$.

(d) $\mathscr{A}_{0}=\cup_{j=1}^{\mathfrak{p}} W^{u}\left(y_{j}^{*, 0}\right)$

Then, $\left\{\mathscr{A}_{\eta}: \eta \in[0,1]\right\}$ is lower semicontinuous at $\eta=0$.

\subsection{Gradient semigroups}

In this section we will consider the gradient semigroups. This class of semigroups appear naturally in several applications and its characteristics allow us to describe with great accuracy the structure of its attractors.

We remember that $y^{*} \in X$ is an equilibrium point for the semigroup $\left\{T(t): t \in \mathbb{T}^{+}\right\}$if the set $\left\{y^{*}\right\}$ is the orbit of a constant global solution; that is, $T(t) y^{*}=y^{*}$, for all $t \in \mathbb{T}^{+}$. We denote by $\mathscr{E}$ the set of equilibrium points of the semigroup $\left\{T(t): t \in \mathbb{T}^{+}\right\}$.

Definition 1.2.1. A semigroup $\left\{T(t): t \in \mathbb{T}^{+}\right\}$is said to be a gradient semigroup if it possesses a Lyapunov function; that is, if there exists a continuous function $V: X \rightarrow \mathbb{R}$ with the following properties:

(i) $\mathbb{T}^{+} \ni t \mapsto V(T(t) x)$ is decreasing for each $x \in X$;

(ii) If $x$ is such that $V(T(t) x)=V(x)$ for all $t \in \mathbb{T}^{+}$, then $x \in \mathscr{E}$.

For gradient semigroups we have the following characterization result:

Lemma 1.2.2. If $\left\{T(t): t \in \mathbb{T}^{+}\right\}$is a gradient semigroup, then $\omega(x)$ is a subset of $\mathscr{E}$ for each $x \in X$. If there exists a global solution $\phi: \mathbb{T} \rightarrow X$ through $x$ then $\alpha_{\phi}(x)$ is a subset of $\mathscr{E}$.

If $\left\{T(t): t \in \mathbb{T}^{+}\right\}$is gradient, has a global attractor $\mathscr{A}$ and $\mathscr{E}$ has only isolated points, then $\mathscr{E}$ is finite and for each $x \in X, \omega(x)$ is an unitary set. In this case, if $x \in \mathscr{A}$ and $\phi: \mathbb{T} \rightarrow \mathscr{A}$ is $a$ global solution through $x$, then $\alpha_{\phi}(x)$ is an unitary set.

In a gradient semigroup the innner structure of the global attractor is known and it is given in the next theorem. 
Theorem 1.2.3. Assume that $\left\{T(t): t \in \mathbb{T}^{+}\right\}$is a gradient semigroup, eventually bounded and asymptotically compact for which the set of equilibrium points $\mathscr{E}$ is bounded. Then $\{T(t): t \in$ $\left.\mathbb{T}^{+}\right\}$has a global attractor $\mathscr{A}=W^{u}(\mathscr{E})$, where

$$
\begin{array}{r}
W^{u}(\mathscr{E}):=\{y \in X: \text { there exists a global solution } \phi(\cdot): \mathbb{T} \rightarrow X \\
\text { through } y(\phi(0)=y) \text { such that } \phi(t) \stackrel{t \rightarrow-\infty}{\longrightarrow} \mathscr{E}\}
\end{array}
$$

is called the unstable set of $\mathscr{E}$. If $\mathscr{E}=\left\{e_{1}^{*}, \cdots, e_{n}^{*}\right\}$ is finite, then $\mathscr{A}=\cup_{i=1}^{n} W^{u}\left(e_{i}^{*}\right)$.

\subsection{Generalized gradient-like semigroups}

We introduce now the concept of generalized gradient-like semigroups using the essential dynamical properties of gradient semigroups with a disjoint family of isolated invariants, and as a particular case, we obtain the gradient-like semigroups (see Carvalho-Langa [10]). We state similar results as in Carvalho-Langa [10], which prove that the properties defining gradient-like semigroups are stable under small perturbations.

Definition 1.3.1. We say that $\Psi=\left\{\Xi_{1}^{*}, \cdots \Xi_{\mathfrak{p}}\right\}$ is a disjoint family of isolated invariants if there exists $\delta>0$ such that $\mathscr{O}_{\delta}\left(\Xi_{i}\right) \cap \mathscr{O}_{\delta}\left(\Xi_{j}\right)=\varnothing, 1 \leqslant i<j \leqslant \mathfrak{p}$, and $\Xi_{i}$ is the maximal invariant set of $\mathscr{O}_{\delta}\left(\Xi_{i}\right):=\left\{z \in X: \operatorname{dist}\left(z, \Xi_{i}\right)<\delta\right\}$.

Let $\left\{T(t): t \in \mathbb{T}^{+}\right\}$be a semigroup with a global attractor $\mathscr{A}$ which contains a disjoint family of isolated invariant sets $\Psi=\left\{\Xi_{1}, \cdots \Xi_{\mathfrak{p}}\right\}$. We define:

Definition 1.3.2. Let $\delta$ be as in Definition 1.3.1 and fix $\epsilon_{0} \in(0, \delta)$. For $\Xi \in \Psi$ and $\epsilon \in\left(0, \epsilon_{0}\right), a$ $\epsilon$-chain from $\Xi$ to $\Xi$ is a sequence $\left\{\Xi_{\ell_{i}}, \cdots, \Xi_{\ell_{k}}\right\} \subset \Psi$, a sequence $t_{1}, \sigma_{1}, \cdots, t_{k}, \sigma_{k}$, with $t_{i}>\sigma_{i}$, $1 \leqslant i \leqslant k, k \leqslant \mathfrak{p}$, along with a sequence of points $u_{i}, 1 \leqslant i \leqslant k$, such that $u_{i} \in \mathscr{O}_{\epsilon}\left(\Xi_{\ell_{i}}\right), T\left(\sigma_{i}\right) u_{i} \notin$ $\mathscr{O}_{\epsilon_{0}}\left(\cup_{i=1}^{k}\left(\Xi_{\ell_{i}}\right)\right)$ and $T\left(t_{i}\right) u_{i} \in \mathscr{O}_{\epsilon}\left(\Xi_{\ell_{i+1}}\right), 1 \leqslant i \leqslant k$, with $\Xi=\Xi_{\ell_{k+1}}=\Xi_{\ell_{1}}$. We say that $\Xi \in \Psi$ is chain recurrent if there exist $\epsilon_{0} \in(0, \delta)$ and $\epsilon$-chains from $\Xi$ to $\Xi$ for each $\epsilon \in\left(0, \epsilon_{0}\right)$.

Definition 1.3.3. Let $\left\{T(t): t \in \mathbb{T}^{+}\right\}$be a semigroup with a global attractor $\mathscr{A}$. We say that $\left\{T(t): t \in \mathbb{T}^{+}\right\}$is gradient-like relative to a disjoint family of isolated invariants $\Psi=\left\{\Xi_{1}, \cdots, \Xi_{\mathfrak{p}}\right\}$ ( r a generalized gradient-like semigroup) if, 
(GG1) For each global solution $\xi: \mathbb{T} \rightarrow X$ in $\mathscr{A}$ there exist $1 \leqslant i, j \leqslant \mathfrak{p}$ such that

$$
\lim _{t \rightarrow-\infty} \operatorname{dist}\left(\xi(t), \Xi_{i}\right)=0 \text { and } \lim _{t \rightarrow \infty} \operatorname{dist}\left(\xi(t), \Xi_{j}\right)=0
$$

(GG2) No element of $\Psi=\left\{\Xi_{1}, \cdots, \Xi_{\mathfrak{p}}\right\}$ is chain recurrent.

We introduce the definitions of unstable and stable sets.

Definition 1.3.4. Let $\left\{T(t): t \in \mathbb{T}^{+}\right\}$be a semigroup. The unstable set of an isolated invariant set $\Xi$ is given by

$$
\begin{array}{r}
W^{\mathrm{u}}(\Xi)=\{\zeta \in X: \text { there exists a global solution } \xi: \mathbb{T} \rightarrow X \\
\text { such that } \left.\xi(0)=\zeta \text { and } \lim _{t \rightarrow-\infty} \operatorname{dist}(\xi(t), \Xi)=0\right\} .
\end{array}
$$

The stable set of an isolated invariant set $\Xi$ for $\left\{T(t): t \in \mathbb{T}^{+}\right\}$is given by

$$
W^{\mathrm{s}}(\Xi)=\left\{\zeta \in X: \lim _{t \rightarrow+\infty} \operatorname{dist}(T(t) \zeta, \Xi)=0\right\}
$$

Given a neighborhood $V$ of $\Xi$, the set of points $y$ of $V$ for which there exists a global solution $\phi_{y}: \mathbb{T} \rightarrow X$ through y such that $\phi_{y}(t) \stackrel{t \rightarrow-\infty}{\longrightarrow} \Xi$ and $\phi_{y}(t) \in V$ for all $t \in \mathbb{T}^{-}$is called $a$ local unstable set of $\Xi$ and it is denoted by $W_{\text {loc }}^{u}(\Xi)$. Analogously we define a local stable set.

The hypotheses (GG1) and (GG2) carry important dynamical properties of a semigroup with a Lyapunov function and a family of isolated invariants (see also Lemmas 1.3 .7 and 1.3.8 below). From (GG1), we have that $\mathscr{A}=\cup_{i=1}^{\mathfrak{p}} W^{u}\left(\Xi_{i}\right)$, since if $x \in \mathscr{A}$ then there exists a global solution $\xi: \mathbb{T} \rightarrow X$ in $\mathscr{A}$ and thus $x \in W^{u}\left(\Xi_{i}\right)$, for some $1 \leqslant i \leqslant \mathfrak{p}$ and conversely, if $x \in W^{u}\left(\Xi_{i}\right)$ for some $1 \leqslant i \leqslant \mathfrak{p}$ then, since $\mathscr{A}$ attracts points, there exists a bounded global solution through $x$, hence $x \in \mathscr{A}$. Also, hypothesis (GG2) says that no collection of orbits can produce a closed path.

The following results are fundamental for the upcoming theory of gradient-like semigroups.

The first lemma is an immediate consequence of the continuity of the semigroups and it ensures that, given an isolated invariant $\Xi$ of a semigroup $\left\{T(t): t \in \mathbb{T}^{+}\right\}$and a point $y$ near $\Xi$, the finite orbit $\gamma_{[0, t]}(t)=\{T(s) y: 0 \leqslant s \leqslant t\}$ remains near $\Xi$ for large values of $t$. 
Lemma 1.3.5. Let $\left\{T(t): t \in \mathbb{T}^{+}\right\}$be a semigroup and $\Xi$ an isolated invariant in $\Psi$. Given $t \in \mathbb{T}^{+}$and $\epsilon>0$ there exists $\delta>0$ such that $\left\{T(s) y: 0 \leqslant s \leqslant t, y \in \mathscr{O}_{\delta}(\Xi)\right\} \subset \mathscr{O}_{\epsilon}(\Xi)$.

The second of them in an important result which is used in several occasions to obtain properties for there semigroups.

Proposition 1.3.6. Assume that $\left\{T(t): t \in \mathbb{T}^{+}\right\}$is an asymptotically compact semigroup. Let $\left\{\sigma_{k}\right\}_{k \in \mathbb{N}}$ be a sequence in $\mathbb{T}^{+}$with $\sigma_{k} \stackrel{k \rightarrow \infty}{\longrightarrow} \infty,\left\{u_{k}\right\}_{k \in \mathbb{N}}$ a bounded sequence in $X$ and, for $\mathbb{J}_{k}=$ $\left\{s \in \mathbb{T}:-\sigma_{k} \leqslant s<\infty\right\}$, define $\xi^{k}: \mathbb{J}_{k} \rightarrow X$ by $\xi^{k}(s)=T\left(s+\sigma_{k}\right) u_{k}, s \in \mathbb{J}_{k}$. If $\left\{T(s) u_{k}: k \in\right.$ $\left.\mathbb{N}, s \in \mathbb{T}^{+}\right\}$is bounded, there exists a bounded global solution $\xi: \mathbb{T} \rightarrow X$ of $\left\{T(t): t \in \mathbb{T}^{+}\right\}$and a subsequence of $\left\{\xi^{k}\right\}_{k \in \mathbb{N}}$ (which we again denote by $\left\{\xi^{k}\right\}_{k \in \mathbb{N}}$ ) such that

$$
\lim _{k \rightarrow \infty} \xi^{k}(s) \rightarrow \xi(s), \quad \forall s \in \mathbb{T}
$$

The third result guarantees that, for a generalized gradient-like semigroup $\left\{T(t): t \in \mathbb{T}^{+}\right\}$, given a bounded set $B$ of $X$ and a neighborhood $\mathscr{O}(\Psi)$ of the disjoint family of isolated invariants $\Psi$, there exists $t_{0}=t_{0}(B, \mathscr{O}(\Psi)) \in \mathbb{T}^{+}$such that all the solutions which begin in points of $B$, must reach $\mathscr{O}(\Psi)$ before the time $t_{0}$.

Lemma 1.3.7. Let $\left\{T(t): t \in \mathbb{T}^{+}\right\}$be a semigroup with a disjoint family of isolated invariants $\Psi=\left\{\Xi_{1}, \cdots, \Xi_{\mathfrak{p}}\right\}$ and a global attractor $\mathscr{A}$. If $\left\{T(t): t \in \mathbb{T}^{+}\right\}$satisfies (GG1), given $\delta<\delta_{0}=$ $\frac{1}{2} \min _{\substack{1 \leqslant i, j \leqslant \mathfrak{p} \\ i \neq j}} \operatorname{dist}\left(\Xi_{i}, \Xi_{j}\right)$ and $B \subset X$ bounded, there exists a $t_{0}=t_{0}(\delta, B)>0$ such that $\left\{T(t) u_{0}: 0 \leqslant\right.$ $\left.t \leqslant t_{0}\right\} \cap \cup_{i=1}^{\mathfrak{p}} \mathscr{O}_{\delta}\left(\Xi_{i}\right) \neq \varnothing$ for all $u_{0} \in B$.

Finally, the fourth result establishes that, if $\left\{T(t): t \in \mathbb{T}^{+}\right\}$is a generalized gradient-like semigroup, given a neighborhood $\mathscr{O}_{2}(\Xi)$ of an invariant set $\Xi \in \Psi$, there exists another neighborhood $\mathscr{O}_{1}(\Xi)$ of $y^{*}$ such that if a solution starts in $\mathscr{O}_{1}(\Xi)$ and leaves $\mathscr{O}_{2}(\Xi)$, then it never returns to $\mathscr{O}_{1}(\Xi)$.

Lemma 1.3.8. Let $\left\{T(t): t \in \mathbb{T}^{+}\right\}$be a generalized gradient-like semigroup. If $\Psi=\left\{\Xi_{1}, \cdots, \Xi_{\mathfrak{p}}\right\}$ is the disjoint family of isolated invariants and $\mathscr{A}$ its global attractor, given $0<\delta<\delta_{0}$, there exists $\delta^{\prime}>0$ such that, iffor some $1 \leqslant i \leqslant \mathfrak{p}, \operatorname{dist}\left(u_{0}, \Xi_{i}\right)<\delta^{\prime}$ and, for some $t_{1}>0, \operatorname{dist}\left(T\left(t_{1}\right) u_{0}, \Xi_{i}\right) \geqslant$ $\delta$, then $\operatorname{dist}\left(T(t) u_{0}, \Xi\right)>\delta^{\prime}$ for all $t \geqslant t_{1}$.

Now we prove that, for a generalized gradient-like semigroup, the $\omega$-limit set of a point lies in one single isolated invariant. We note that condition (GG1) is imposed only for solutions in the attractor. 
Lemma 1.3.9. Assume that $\left\{T(t): t \in \mathbb{T}^{+}\right\}$is a generalized gradient-like semigroup with a disjoint family of isolated invariants $\Psi=\left\{\Xi_{1}, \cdots, \Xi_{\mathfrak{p}}\right\}$ and a global attractor $\mathscr{A}$. Given $u \in X$ there exists $\Xi_{i} \in \Psi$ such that

$$
T(t) u \stackrel{t \rightarrow \infty}{\longrightarrow} \Xi_{i} .
$$

Proof: It follows from Lemma 1.3 .8 that, given $\delta \in\left(0, \delta_{0}\right)$ there exists $\delta^{\prime} \in(0, \delta)$ such that $d\left(\nu, \Xi_{i}\right)<\delta^{\prime}$ and if for some $t_{\nu, \delta}>0, d\left(T\left(t_{v, \delta}\right) \nu, \Xi_{i}\right) \geqslant \delta$, then $\operatorname{dist}\left(T(t) \nu, \Xi_{i}\right)>\delta^{\prime}$ for all $t \geqslant t_{\nu, \delta}$. On the other hand, since $\gamma^{+}(u)$ is bounded, it follows from Lemma 1.3.7that, given $\delta^{\prime}$ there exists a $t_{\delta^{\prime}}=t_{\delta^{\prime}}\left(\gamma^{+}(u)\right) \in \mathbb{T}$ such that, for each $v \in \gamma^{+}(u)$,

$$
\left\{T(t) v: 0 \leqslant t \leqslant t_{\delta^{\prime}}\right\} \cap \cup_{i=1}^{\mathfrak{p}} \mathscr{O}_{\delta^{\prime}}\left(\Xi_{i}\right) \neq \varnothing
$$

From the fact that $\Psi$ is finite follows that there exist an $\Xi_{j} \in \Psi$ and, for each $\delta \in\left(0, \delta_{0}\right)$, a $s_{\delta} \in \mathbb{T}^{+}$such that $T(s) u \in \mathscr{O}_{\delta}\left(\Xi_{j}\right)$ for all $s \geqslant s_{\delta}$. This completes the proof.

Definition 1.3.10. Let $\left\{T(t): t \in \mathbb{T}^{+}\right\}$be a semigroup with a disjoint family of isolated invariants $\Psi=\left\{\Xi_{1}, \cdots, \Xi_{\mathfrak{p}}\right\}$ and a global attractor $\mathscr{A}$. A homoclinic structure in $\mathscr{A}$ is a set $\left\{\Xi_{\ell_{1}}, \cdots, \Xi_{\ell_{k}}\right\} \subset \Psi$ along with a set of global solutions $\left\{\xi^{(i)}: \mathbb{T} \rightarrow X, 1 \leqslant i \leqslant k\right\}$ in $\mathscr{A}$ such that, taking $\Xi_{\ell_{k+1}} \doteq \xi_{\ell_{1}}$,

$$
\lim _{t \rightarrow-\infty} \xi^{(i)}(t)=\Xi_{\ell_{i}}, \quad \lim _{t \rightarrow+\infty} \xi^{(i)}(t)=\Xi_{\ell_{i+1}}, \quad 1 \leqslant i \leqslant k
$$

Now we can prove the following result, which relates (GG1) and (GG2) to the non-existence of homoclinic structures.

Lemma 1.3.11. Let $\left\{T(t): t \in \mathbb{T}^{+}\right\}$be a semigroup with a disjoint family of isolated invariants $\Psi=\left\{\Xi_{1}, \cdots, \Xi_{\mathfrak{p}}\right\}$ and a global attractor $\mathscr{A}$. If $\left\{T(t): t \in \mathbb{T}^{+}\right\}$satisfies (GG1), then (GG2) is satisfied if and only if $\mathscr{A}$ does not possess homoclinic structures.

Proof: If $\mathscr{A}$ has a homoclinic structure and $\Xi$ is an isolated invariant in this structure it is easy to see that $\Xi$ is chain recurrent.

On the other hand, if $\Xi \in \Psi$ is chain recurrent, there exist $\delta<\delta_{0},\left\{\Xi_{\ell_{1}}, \cdots, \Xi_{\ell_{r+1}}\right\} \subset \Psi$ and for each $\mathbb{N} \ni k>\frac{1}{\delta}$, points $y_{1}^{k}, \cdots, y_{r+1}^{k}, t_{1}^{k}>\tau_{1}^{k}, \cdots, t_{r}^{k}>\tau_{r}^{k}$ such that

$$
\operatorname{dist}\left(y_{i}^{k}, \Xi_{\ell_{i}}\right)<\frac{1}{k}, \operatorname{dist}\left(T\left(\tau_{i}^{k}\right) y_{i}^{k}, \Psi\right)>\delta, \operatorname{dist}\left(T\left(t_{i}^{k}\right) y_{i}^{k}, \Xi_{\ell_{i+1}}\right)<\frac{1}{k}, 1 \leqslant i \leqslant r .
$$


Choose $\sigma_{i}^{k}>0$ such that $\operatorname{dist}\left(T\left(\sigma_{i}^{k}\right) y_{i}^{k}, \Xi_{\ell_{i}}\right) \geqslant \delta$ and $\operatorname{dist}\left(T(t) y_{i}^{k}, \Xi_{\ell_{i}}\right)<\delta$, for all $0 \leqslant t<\sigma_{i}^{k}$. From Lemma1.3.5it follows that $\sigma_{i}^{k} \stackrel{k \rightarrow \infty}{\longrightarrow}+\infty$.

For $t \in\left[-\sigma_{i}^{k}, \infty\right)$ let $\xi^{i, k}(t)=T\left(\sigma_{i}^{k}+t\right) y_{i}^{k}$.

From Proposition 1.3.6, there exists a global solution $\xi^{(i)}: \mathbb{T} \rightarrow X$. Since each $\xi^{(i)}$ must converge to an isolated invariant as $t \rightarrow+\infty$ and as $t \rightarrow-\infty$ and since $\xi^{(i)}(t) \in \mathscr{O}_{\delta}\left(\Xi_{\ell_{i}}\right)$ for all $t<0$ we have that $\xi^{(i)}(t) \rightarrow \Xi_{\ell_{i}}$ as $t \rightarrow-\infty$. We can assume that, between $-\sigma_{k}^{i}$ and $t_{k}^{i}-\sigma_{k}^{i}$, the solution $\xi^{k, i}$ remains away from $\Psi \backslash\left\{\Xi_{\ell_{i}}, \Xi_{\ell_{i+1}}\right\}$ otherwise we could insert more points in the $\epsilon$-chains until this holds. From Lemma $1.3 .8, \xi^{(i)}(t) \stackrel{t \rightarrow \infty}{\longrightarrow} \Xi_{\ell_{i+1}}$.

The set $\left\{\Xi_{\ell_{1}}, \cdots, \Xi_{\ell_{k}}\right\} \subset \Psi$ and the set of global solutions $\left\{\xi^{(i)}: \mathbb{T} \rightarrow X, 1 \leqslant i \leqslant k\right\}$ are such that,

$$
\lim _{t \rightarrow-\infty} \operatorname{dist}\left(\xi_{n}^{(i)}, \Xi_{\ell_{i}}\right)=0, \quad \lim _{t \rightarrow+\infty} \operatorname{dist}\left(\xi_{n}^{(i)}, \Xi_{\ell_{i+1}}\right)=0, \quad 1 \leqslant i \leqslant k,
$$

with $\Xi_{\ell_{k+1}} \doteq \Xi_{\ell_{1}}$. Therefore $\mathscr{A}$ has a homoclinic structure.

Corollary 1.3.12. If $\left\{T(t): t \in \mathbb{T}^{+}\right\}$is a generalized gradient-like semigroup and $\mathscr{A}$ its attractor, there exist isolated invariants $\Xi_{\alpha}$ and $\Xi_{\omega}$ such that $\Xi_{\alpha}$ has a trivial stable set in $\mathscr{A}$; that is, $W_{\mathscr{A}}^{s}\left(\Xi_{\alpha}\right)=\Xi_{\alpha}$ where

$$
W_{\mathscr{A}}^{s}\left(\Xi_{\alpha}\right) \doteq\left\{y \in \mathscr{A}: \text { such that } T(t) y \stackrel{t \rightarrow \infty}{\longrightarrow} \Xi_{\alpha}\right\}
$$

and $\Xi_{\omega}$ has a trivial unstable set; that is, $W^{u}\left(\Xi_{\omega}\right)=\Xi_{\omega}$.

In the following we consider a family of semigroups $\left\{T_{\eta}(t): t \in \mathbb{T}^{+}\right\}_{\eta \in[0,1]}$. We denote by $\mathscr{E}_{\eta}$ the set of equilibrium points of $\left\{T_{\eta}(t): t \in \mathbb{T}^{+}\right\}$for each $\eta \in[0,1]$.

Definition 1.3.13. We say that a family of semigroups $\left\{T_{\eta}(t): t \in \mathbb{T}^{+}\right\}_{\eta \in[0,1]}$ is collective asymptotically compact at $\eta=0$ if, given sequences $\left\{\eta_{k}\right\}_{k \in \mathbb{N}}$ with $\eta_{k} \stackrel{k \rightarrow \infty}{\longrightarrow} 0$, a bounded sequence $\left\{u_{k}\right\}_{k \in \mathbb{N}}$ in $X$ and a sequence $\left\{t_{k}\right\}_{k \in \mathbb{N}}$ in $\mathbb{T}^{+}$with $t_{k} \stackrel{k \rightarrow \infty}{\longrightarrow} \infty$ and $\left\{T_{\eta_{k}}\left(t_{k}\right) u_{k}: k \in \mathbb{N}\right\}$ bounded, then $\left\{T_{\eta_{k}}\left(t_{k}\right) u_{k}: k \in \mathbb{N}\right\}$ is precompact.

Now we state the result of stability of the generalized gradient-like semigroups under some kind of perturbations, as done in [10]. This allows us to characterize the attractors of semigroups given as a small perturbation of generalized gradient-like semigroups, and if we perturbe again the resulting semigroup we are still able to give a characterization of the attractors. 
Theorem 1.3.14. Let $\left\{T_{\eta}(t): t \in \mathbb{T}^{+}\right\}, \eta \in[0,1]$, be a continuous and collective asymptotically compact family of semigroups at $\eta=0$. Suppose that

(a) $\left\{T_{\eta}(t): t \in \mathbb{T}^{+}\right\}$has a global attractor $\mathscr{A}_{\eta}$ for each $\eta \in[0,1]$ and $\cup_{\eta \in[0,1]} \mathscr{A}_{\eta}$ is bounded.

(b) There exists $\mathfrak{p} \in \mathbb{N}$ such that $\mathscr{A}_{\eta}$ has $\mathfrak{p}$ isolated invariant sets $\Psi_{\eta}=\left\{\Xi_{1, \eta}, \cdots, \Xi_{\mathfrak{p}, \eta}\right\}$ for all $\eta \in[0,1]$ and $\sup _{1 \leqslant i \leqslant p}\left[\mathrm{~d}_{H}\left(\Xi_{i, \eta}, \Xi_{i, 0}\right)+\mathrm{d}_{H}\left(\Xi_{i, 0}, \Xi_{i, \eta}\right)\right] \stackrel{\eta \rightarrow 0}{\longrightarrow} 0$.

(c) $\left\{T_{0}(t): t \in \mathbb{T}^{+}\right\}$is a gradient-like semigroup relative to the disjoint family of isolated invariants $\Psi_{0}=\left\{\Xi_{1,0}, \cdots, \Xi_{\mathfrak{p}, 0}\right\}$.

Then there exists $\eta_{0}>0$ such that, for all $\eta \leqslant \eta_{0},\left\{T_{\eta}(t): t \in \mathbb{T}^{+}\right\}$is a gradient-like semigroup relative to the disjoint family of isolated invariants $\Psi_{\eta}$. Consequently, there exists $\eta_{0}>0$ such that

$$
\mathscr{A}_{\eta}=\cup_{i=1}^{\mathfrak{p}} W^{u}\left(\Xi_{i, \eta}\right), \forall \eta \in\left[0, \eta_{0}\right]
$$

In the case where each $\Xi_{i}$ consists of a single point; that is $\Xi_{i}=\left\{y_{i}^{*}\right\}$, it is clear that each $y_{i}^{*}$ is an equilibrium point of the semigroup $\left\{T(t): t \in \mathbb{T}^{+}\right\}$and we say simply that the semigroup is gradient-like.

\subsection{Gradient-like semigroups are gradient semigroups}

The Fundamental Theorem on Dynamical Systems, suggested in [34] from the results in [16], establishes that in any compact metric space an attractor can be described by isolated invariant sets and the connections between them. In the terminology of [16], this decomposition is called Morse decomposition (see Definition 1.4.8), and it was considered in different contexts, in the case of groups by [16] and in the case of semigroups by [44], or even in a topological space, compact or not, in [21, 35, 36]. For a complete understanding of the subject, we refer to [1], where all these results are proven in detail.

\subsubsection{Morse decomposition of gradient-like semigroups}

Let $X$ be a metric space with metric $d: X \times X \rightarrow \mathbb{R}^{+}$. In what follows we present the notion of a Morse decomposition for a global attractor $\mathscr{A}$ of a gradient-like semigroup $\{T(t): t \geqslant 0\}$. We begin with the notion of attractor-repeller pair. 
Definition 1.4.1. Let $\{T(t): t \geqslant 0\}$ be a semigroup with a global attractor $\mathscr{A}$. We say that a non-empty subset $\Xi$ of $\mathscr{A}$ is a local attractor if there exists $\epsilon>0$ such that $\omega\left(\mathscr{O}_{\epsilon}(\Xi)\right)=\Xi$. The repeller $\Xi^{*}$ associated with the local attractor $\Xi$ is the set defined by

$$
\Xi^{*}=\{x \in \mathscr{A}: \omega(x) \cap \Xi=\varnothing\} .
$$

The pair $\left(\Xi, \Xi^{*}\right)$ is called an attractor-repeller pair of $\{T(t): t \geqslant 0\}$.

Remark 1.4.2. Note that, if $\Xi$ is a local attractor, then $\Xi^{*}$ is closed, invariant and $\Xi \cap \Xi^{*}=\varnothing$.

Observe that $\Xi$ is a local attractor if and only if it is invariant and attracts $\mathscr{O}_{\epsilon}(\Xi)$ for some $\epsilon>0$. Also, the above definition differ a little from the usual definition since we require that the local attractor attracts a neighborhood of $\Xi$ in $X$ and not in $\mathscr{A}$ as in [16, 44]. We prove that both definitions coincide.

Lemma 1.4.3. Let $\{T(t): t \geqslant 0\}$ be a semigroup in $X$ with global attractor $\mathscr{A}$. If $\Xi$ is a compact and invariant set for $\{T(t): t \geqslant 0\}$ and there exists $\epsilon>0$ such that $\Xi$ attracts $\mathscr{O}_{\epsilon}(\Xi) \cap$ $\mathscr{A}$ then, given $\delta>0$ there exists $\delta^{\prime}>0$ such that $\gamma^{+}\left(\mathscr{O}_{\delta^{\prime}}(\Xi)\right) \subset \mathscr{O}_{\delta}(\Xi)$, where $\gamma^{+}\left(\mathscr{O}_{\delta^{\prime}}(\Xi)\right)=$ $\bigcup_{x \in \mathscr{O}_{\delta^{\prime}}(\Xi)} \bigcup_{t \geqslant 0} T(t) x$.

Proof: Given $0<\delta<\epsilon$ if there is no $\delta^{\prime}>0$ such that $\gamma^{+}\left(\mathscr{O}_{\delta^{\prime}}(\Xi)\right) \subset \mathscr{O}_{\delta}(\Xi)$, there exist $x \in \Xi$, $X \ni x_{n} \stackrel{n \rightarrow \infty}{\longrightarrow} x$ and $\mathbb{R} \ni t_{n} \stackrel{n \rightarrow \infty}{\longrightarrow} \infty$ such that $d\left(T\left(t_{n}\right) x_{n}, \Xi\right)=\delta$ and $T(t) x_{n} \in \mathscr{O}_{\delta}(\Xi), t \in\left[0, t_{n}\right]$. Since $\{T(t): t \geqslant 0\}$ has a global attractor, it is not hard to see that there exists a global solution $\xi: \mathbb{R} \rightarrow X$ such that $\xi_{n}:\left[-t_{n}, \infty\right) \rightarrow X$ given by $\xi_{n}(t)=T\left(t_{n}+t\right) x_{n}$ satisfying $\xi_{n}(t) \stackrel{n \rightarrow \infty}{\longrightarrow} \xi(t)$ for each $t \in \mathbb{R}$. Clearly $\xi(t) \in \overline{\mathscr{O}_{\delta}(\Xi)} \cap \mathscr{A} \subset \mathscr{O}_{\epsilon}(\Xi) \cap \mathscr{A}$ for all $t \leqslant 0, d(\xi(0), \Xi)=\delta$, and consequently $\Xi$ cannot attract $\mathscr{O}_{\epsilon}(\Xi) \cap \mathscr{A}$.

Lemma 1.4.4. If $\{T(t): t \geqslant 0\}$ is a semigroup in $X$ with a global attractor $\mathscr{A}$ and $\left.S(t) \doteq T(t)\right|_{\mathscr{A}}$, clearly $\{S(t): t \geqslant 0\}$ is a semigroup in the metric space $\mathscr{A}$. If $\Xi$ is a local attractor for $\{S(t): t \geqslant 0\}$ in the metric space $\mathscr{A}$ and $K$ is a compact subset of $\mathscr{A}$ such that $K \cap \Xi^{*}=\varnothing$, then $\Xi$ attracts $K$. Moreover $\Xi$ is a local attractor for $\{T(t): t \geqslant 0\}$ in $X$.

Proof: Let $K$ be a compact subset of $\mathscr{A}$ such that $K \cap \Xi^{*}=\varnothing$. If $\Xi=\omega\left(\mathscr{O}_{\epsilon}(\Xi) \cap \mathscr{A}\right)$ does not attract $K$ and $0<\delta<\epsilon$, there exist a $\delta^{\prime} \in(0, \delta)$, a sequence $\left\{t_{n}\right\}_{n \in \mathbb{N}}$ in $\mathbb{T}^{+}$with $t_{n} \stackrel{n \rightarrow \infty}{\longrightarrow} \infty, x \in K$ 
and $K \ni x_{n} \stackrel{n \rightarrow \infty}{\longrightarrow} x$ such that $d\left(T(t) x_{n}, \Xi\right) \geqslant \delta^{\prime}, 0 \leqslant t \leqslant t_{n}$. This implies that $d(T(t) x, \Xi) \geqslant \delta^{\prime}$ for all $t \geqslant 0$ and, consequently, $\omega(x) \cap \Xi=\varnothing$ and therefore $x \in \Xi^{*}$ which is a contradiction.

For the remaining part of the result, note that, from Lemma 1.4.3, there exists a $\delta^{\prime} \in(0, \epsilon)$ such that $\omega\left(\mathscr{O}_{\delta^{\prime}}(\Xi)\right) \subset \mathscr{O}_{\epsilon}(\Xi) \cap \mathscr{A}$ and hence $\omega\left(\mathscr{O}_{\delta^{\prime}}(\Xi)\right) \cap \Xi^{*}=\varnothing$. From the invariance of $\omega\left(\mathscr{O}_{\delta^{\prime}}(\Xi)\right)$ and from the property that $\Xi$ attracts $\mathscr{O}_{\epsilon}(\Xi) \cap \mathscr{A}$, we have that $\omega\left(\mathscr{O}_{\delta^{\prime}}(\Xi)\right) \subset \Xi$. Since $\omega\left(\mathscr{O}_{\delta^{\prime}}(\Xi)\right)$ attracts $\mathscr{O}_{\delta^{\prime}}(\Xi)$ the result follows.

Lemma 1.4.5. Let $\{T(t): t \geqslant 0\}$ be a semigroup in $X$ with a global attractor $\mathscr{A}$ and an attractorrepeller pair $\left(\Xi, \Xi^{*}\right)$.

1. A global solution $\xi: \mathbb{R} \rightarrow X$ of $\{T(t): t \geqslant 0\}$ with the property that $\overline{\xi(\mathbb{R})} \cap \Xi^{*} \neq \varnothing$ must $\operatorname{satisfy} d\left(\xi(t), \Xi^{*}\right) \stackrel{t \rightarrow-\infty}{\longrightarrow} 0$.

2. A global solution $\xi: \mathbb{R} \rightarrow X$ of $\{T(t): t \geqslant 0\}$ with the property that $\xi(t) \in \mathscr{O}_{\delta}\left(\Xi^{*}\right)$ for all $t \leqslant 0$ and some $\delta>0$ such that $\mathscr{O}_{\delta}\left(\Xi^{*}\right) \cap \Xi=\varnothing$ must satisfy $d\left(\xi(t), \Xi^{*}\right) \stackrel{t \rightarrow-\infty}{\longrightarrow} 0$.

Proof: If the conclusion of $\mathbf{1}$ is not true, there exist a $\delta^{\prime}>0$ and a sequence $t_{n} \stackrel{n \rightarrow \infty}{\longrightarrow} \infty$ such that $d\left(\xi\left(-t_{n}\right), \Xi^{*}\right) \geqslant \delta^{\prime}$ and, for some $t \in\left[-t_{n}-1,-t_{n}\right), d\left(\xi(t), \Xi^{*}\right)<\delta^{\prime}$. This contradicts the fact that $\Xi$ attracts the compact set $K=\left\{z \in \mathscr{A}: d\left(z, \Xi^{*}\right) \geqslant \delta^{\prime}\right\}$ of $\mathscr{A}$.

To prove 2 we observe that if $\overline{\xi(\mathbb{R})} \cap \Xi^{*}=\varnothing$, from Lemma 1.4.4 we have that $\overline{\xi(\mathbb{R})} \subset \Xi$ which lead us to a contradiction. On the other hand, if $\overline{\xi(\mathbb{R})} \cap \Xi^{*} \neq \varnothing$, it follows from $\mathbf{1}$ that $d\left(\xi(t), \Xi^{*}\right) \stackrel{t \rightarrow-\infty}{\longrightarrow} 0$. This completes the proof.

Lemma 1.4.6. Let $\{T(t): t \geqslant 0\}$ be a semigroup in $X$ with global attractor $\mathscr{A}$ and an attractorrepeller pair $\left(\Xi, \Xi^{*}\right)$. If $\xi: \mathbb{R} \rightarrow X$ is a bounded global solution for $\{T(t): t \geqslant 0\}$ through $x \notin$ $\Xi \cup \Xi^{*}$, then $\xi(t) \stackrel{t \rightarrow \infty}{\longrightarrow} \Xi$ and $\xi(t) \stackrel{t \rightarrow-\infty}{\longrightarrow} \Xi^{*}$. Moreover, if $x \in X \backslash \mathscr{A}$ then, $T(t) x \stackrel{t \rightarrow \infty}{\longrightarrow} \Xi \cup \Xi^{*}$.

Proof: Since $x \notin \Xi^{*}$ we have that $\omega(x) \cap \Xi$ is non-empty and, from the fact that $\Xi$ is a local attractor, we have that $\xi(t) \stackrel{t \rightarrow \infty}{\longrightarrow} \Xi$. On the other hand, if $\xi(t)$ does not converge to $\Xi^{*}$ as $t \rightarrow-\infty$ we divide the proof in two steps: if $\overline{\xi(\mathbb{R})} \cap \Xi^{*}=\varnothing$, then $\xi(\mathbb{R})$ is invariant and, from Lemma 1.4.4, is attracted by $\Xi$ which is a contradiction. Thus, $\overline{\xi(\mathbb{R})} \cap \Xi^{*}$ is non-empty and from Lemma 1.4.5 we have that $d\left(\xi(t), \Xi^{*}\right) \stackrel{t \rightarrow-\infty}{\longrightarrow} 0$.

For $x \in X \backslash \mathscr{A}$ we prove that $T(t) x \stackrel{t \rightarrow \infty}{\longrightarrow} \Xi \cup \Xi^{*}$. If $\overline{\gamma^{+}(x)} \cap \Xi \neq \varnothing$ we have that $T(t) x \stackrel{t \rightarrow \infty}{\longrightarrow} \Xi$. On the other hand, if there exists $\delta>0$ with $\gamma^{+}(z) \cap \mathscr{O}_{\delta}(\Xi)=\varnothing$ we state that $T(t) x \stackrel{t \rightarrow \infty}{\longrightarrow} \Xi^{*}$. If 
our statement is false, there exist a $v>0$ and a sequence $t_{n} \stackrel{n \rightarrow \infty}{\longrightarrow} \infty$ such that $d\left(T\left(t_{n}\right) x, \Xi^{*}\right) \geqslant$ $v$. Considering the sequence of functions $\xi_{n}:\left[-t_{n}, \infty\right) \rightarrow X$ defined by $\xi_{n}(t)=T\left(t+t_{n}\right) x$, $t \geqslant-t_{n}$, we construct a global solution $\xi: \mathbb{R} \rightarrow \mathscr{A}$ such that $d\left(\xi(0), \Xi^{*}\right) \geqslant v$ and $d(\xi(t), \Xi) \geqslant \delta$ for all $t \in \mathbb{R}$. Therefore $\omega(\xi(0)) \cap \Xi=\varnothing$ and $\xi(0) \in \Xi^{*}$ which is a contradiction.

Corollary 1.4.7. If $\{T(t): t \geqslant 0\}$ is a semigroup in $X$ with a global attractor $\mathscr{A}$ and $\left(\Xi, \Xi^{*}\right)$ is an attractor-repeller pair for $\{T(t): t \geqslant 0\}$, then $\{T(t): t \geqslant 0\}$ is a gradient-like semigroup relative to the disjoint family of isolated invariants $\left\{\Xi, \Xi^{*}\right\}$.

With this we can begin the construction of a Morse decomposition for the attractor of a gradient-like semigroup relative to an invariant family of isolated invariant sets. We begin fixing the definition of Morse decomposition we will use here.

Definition 1.4.8. Given an increasing family $\varnothing=A_{0} \subset A_{1} \subset \cdots \subset A_{n}=\mathscr{A}$, of $n+1$ local attractors, define $\Xi_{j}:=A_{j} \cap A_{j-1}^{*}$, for $j=1, \cdots, n$. The ordered $n$-tuple $\Psi \doteq\left\{\Xi_{1}, \Xi_{2}, \cdots, \Xi_{n}\right\}$ is called $a$ Morse decomposition of $\mathscr{A}$.

In what follows, our goal is to show that if $\{T(t): t \geqslant 0\}$ is a gradient-like semigroup relative to a disjoint family of isolated invariants $\Xi=\left\{\Xi_{1}, \cdots, \Xi_{n}\right\}$ and with a global attractor $\mathscr{A}$, then some reordering of $\Xi$ is a Morse decomposition of $\mathscr{A}$. The next result plays an important role in this process and its proof is straightforward.

Lemma 1.4.9. Let $\{T(t): t \geqslant 0\}$ be a semigroup with global attractor $\mathscr{A}$ and $\Xi \subset \mathscr{A}$ an isolated invariant set. Then $\Xi$ is a local attractor if and only if $W^{\mathrm{u}}(\Xi)=\Xi$.

Lemma 1.4.10. Let $\{T(t): t \geqslant 0\}$ be a gradient-like semigroup relative to a disjoint family of isolated invariants $\Psi=\left\{\Xi_{1}, \cdots, \Xi_{n}\right\}$. Then there exists $1 \leqslant k \leqslant n$ such that $\Xi_{k}$ is a local attractor for $\{T(t): t \geqslant 0\}$ in $X$.

Let $\{T(t): t \geqslant 0\}$ be a gradient-like semigroup relative to a disjoint family of isolated invariants $\Psi=\left\{\Xi_{1}, \cdots, \Xi_{n}\right\}$. If (after a possible reordering) $\Xi_{1}$ is a local attractor for $\{T(t): t \geqslant 0\}$ and

$$
\Xi_{1}^{*}=\left\{a \in \mathscr{A}: \omega(a) \cap \Xi_{1}=\varnothing\right\},
$$

then each $\Xi_{i}, i>1$ is contained in $\Xi_{1}^{*}$ and for each $a \in \mathscr{A} \backslash\left\{\Xi_{1} \cup \Xi_{1}^{*}\right\}$ and global solution $\phi: \mathbb{R} \rightarrow \mathscr{A}$ with $\phi(0)=a$ we have that

$$
\Xi_{1}^{*} \stackrel{t \rightarrow-\infty}{\longleftarrow} \phi_{j}(t) \stackrel{t \rightarrow \infty}{\longrightarrow} \Xi_{1}
$$


Considering the restriction $T_{1}(t)$ of $T(t)$ to $\Xi_{1,0}^{*} \doteq \Xi_{1}^{*}$ we have that $T_{1}(t)$ is a gradient-like semigroup $\Xi_{1}^{*}$ relative to the disjoint family of isolated invariants $\left\{\Xi_{2}, \cdots, \Xi_{n}\right\}$ and we can assume, without loss of generality, that $\Xi_{2}$ is a local attractor for $\left\{T_{1}(t): t \geqslant 0\right\}$ in $\Xi_{1}^{*}$. If $\Xi_{2,1}^{*}$ is the repeller associated with the isolated invariant set $\Xi_{2}$ of $\left\{T_{1}(t): t \geqslant 0\right\}$ in $\Xi_{1}^{*}$ we can proceed and consider the restriction $\left\{T_{2}(t): t \geqslant 0\right\}$ of the semigroup $\left\{T_{1}(t): t \geqslant 0\right\}$ to $\Xi_{2,1}^{*}$ and $\left\{T_{2}(t): t \geqslant 0\right\}$ is a gradient-like semigroup in $\Xi_{2,1}^{*}$ relative to the family of isolated invariants $\left\{\Xi_{3}, \cdots, \Xi_{n}\right\}$.

Proceeding with this reasoning, after a finite number of steps, we obtain a reordering of $\left\{\Xi_{1}, \cdots, \Xi_{n}\right\}$ in such a way that $\Xi_{j}$ is a local attractor for the restriction of $\{T(t): t \geqslant 0\}$ to $\Xi_{j, j-1}^{*}$ $\left(\Xi_{0,-1}^{*} \doteq \mathscr{A}\right)$.

With this construction, if a global solution $\xi: \mathbb{R} \rightarrow \mathscr{A}$ satisfies

$$
\Xi_{\ell} \stackrel{t \rightarrow-\infty}{\longleftarrow} \xi(t) \stackrel{t \rightarrow \infty}{\longrightarrow} \Xi_{k}
$$

then $\ell \geqslant k$.

In order to see this, firstly we observe that if $\left(\Xi, \Xi^{*}\right)$ is an attractor-repeller pair, any global solution $\zeta: \mathbb{R} \rightarrow X$ with $\xi(0) \in \Xi^{*}$ satisfies $\xi(t) \in \Xi^{*}$ for all $t \in \mathbb{R}$. From the convergence of $\xi(\cdot)$ to $\Xi_{k}$, necessarily $\xi(0) \in \Xi_{k-1, k-2}^{*}$. But $\Xi_{k-1, k-2}^{*}$ in invariant and contains only the isolated invariants $\left\{\Xi_{k}, \Xi_{k+1}, \ldots, \Xi_{n}\right\}$. Thus it follows immediately that $\ell \geqslant k$.

We prove now that this reordering of $\left\{\Xi_{1}, \cdots, \Xi_{n}\right\}$ (which we denote the same way) is a Morse decomposition for $\mathscr{A}$ with a sequence carefully chosen $A_{0} \subset A_{1} \subset A_{2} \subset \ldots \subset A_{n}$ of local attractors.

Define $A_{0}=\varnothing, A_{1}=\Xi_{1}$ and for $j=2,3, \cdots, n$

$$
A_{j}=A_{j-1} \cup W^{\mathrm{u}}\left(\Xi_{j}\right)=\cup_{i=1}^{j} W^{\mathrm{u}}\left(\Xi_{i}\right)
$$

It is clear that $A_{n}=\mathscr{A}$ and each $A_{j}$ is compact.

Theorem 1.4.11. Let $\{T(t): t \geqslant 0\}$ be a gradient-like semigroup relative to a disjoint family of isolated invariants $\Psi=\left\{\Xi_{1}, \cdots, \Xi_{n}\right\}$ reordered in such a way that $\Xi_{j}$ is a local attractor for the restriction of $\{T(t): t \geqslant 0\}$ to $\Xi_{j-1, j-2}^{*}$. Then $A_{j}$ defined in (1.4.2) is a local attractor for $\{T(t): t \geqslant 0\}$ in $X$,

$$
\Xi_{j}=A_{j} \cap A_{j-1}^{*}
$$

and $\Psi$ is a Morse decomposition of $\mathscr{A}$. 
Proof: From Lemma 1.4.4, is sufficient to prove that $A_{j}=A_{j-1} \cup W^{\mathrm{u}}\left(\Xi_{j}\right)$ is a local attractor for $\{T(t): t \geqslant 0\}$ restricted to the global attractor $\mathscr{A}$.

Choose $d>0$ such that $\mathscr{O}_{d}\left(\cup_{i=1}^{j} W^{u}\left(\Xi_{i}\right)\right) \cap\left(\cup_{i=j+1}^{n} \Xi_{i}\right)=\varnothing$. If there exist $\delta<d$ and $\delta^{\prime}<\delta$ such that $\gamma^{+}\left(\mathscr{O}_{\delta^{\prime}}\left(A_{j}\right) \cap \mathscr{A}\right) \subset \mathscr{O}_{\delta}\left(A_{j}\right) \cap \mathscr{A}$, then $\omega\left(\mathscr{O}_{\delta^{\prime}}\left(A_{j}\right) \cap \mathscr{A}\right)$ attracts $\mathscr{O}_{\delta^{\prime}}\left(A_{j}\right) \cap \mathscr{A}$ and (since $\left.\omega\left(\mathscr{O}_{\delta^{\prime}}\left(A_{j}\right)\right) \cap \mathscr{A}\right)$ is invariant) is contained in $A_{j}$, proving that $A_{j}$ is a local attractor in $\mathscr{A}$. If this is not the case, there exist a sequence $\left\{x_{k}\right\}$ in $\mathscr{A} \backslash A_{j}$ with $d\left(x_{k}, A_{j}\right) \stackrel{k \rightarrow \infty}{\longrightarrow} 0$, for each $x_{k}$ a global solution $\xi_{k}: \mathbb{R} \rightarrow \mathscr{A}$ through $x_{k}$ and a sequence $t_{k} \stackrel{k \rightarrow \infty}{\longrightarrow} \infty$, such that $d\left(\xi_{k}(t), A_{j}\right) \leqslant \delta$ for all $t \in\left[0, t_{k}\right]$ and $d\left(\xi_{k}\left(t_{k}\right), A_{j}\right)=\delta$. In this way, we construct a global solution $\xi: \mathbb{R} \rightarrow \mathscr{A}$ such that $d\left(\xi(t), A_{j}\right) \leqslant \delta$ for all $t \leqslant 0$ and $d\left(\xi(0), A_{j}\right)=\delta$. This gives us a contradiction.

To prove that $\Xi_{j}=A_{j} \cap A_{j-1}^{*}$ note that $A_{j} \supset \bigcup_{i=1}^{j} \Xi_{i}$ (see (1.4.2)) and $A_{j-1}^{*}=\{z \in \mathscr{A}$ : $\left.\omega(z) \cap A_{j-1}=\varnothing\right\} \supset \bigcup_{i=j}^{n} \Xi_{i}$. Therefore, given $z \in A_{j} \cap A_{j-1}^{*}$ we have that a global solution $\xi: \mathbb{R} \rightarrow \mathscr{A}$ through $z$ must satisfy

$$
\Xi_{\ell} \stackrel{t \rightarrow-\infty}{\longleftarrow} \xi(t) \stackrel{t \rightarrow \infty}{\longrightarrow} \Xi_{k}
$$

with $k \leqslant \ell \leqslant j$ (from the fact that $z \in A_{j}$ ) and $j \leqslant k \leqslant \ell$ (from the fact that $z \in A_{j}^{*}$ ). This and from the fact that $\{T(t): t \geqslant 0\}$ is a gradient-like semigroup relative to the disjoint family of invariants $\left\{\Xi_{1}, \cdots, \Xi_{n}\right\}$, we obtain that $z \in \Xi_{j}$. Thus $A_{j} \cap A_{j-1}^{*} \subset \Xi_{j}$. The other inclusion is straightforward from the definition of $A_{j}$ and $A_{j-1}^{*}$.

Proposition 1.4.12. Let $\{T(t): t \geqslant 0\}$ be a gradient-like semigroup relative to the disjoint family of isolated invariants $\Psi=\left\{\Xi_{1}, \cdots, \Xi_{n}\right\}$ reordered in such a way that it is a Morse decomposition of $\mathscr{A}$. Then,

$$
\bigcap_{j=0}^{n}\left(A_{j} \cup A_{j}^{*}\right)=\bigcup_{j=1}^{n} \Xi_{j} .
$$

Proof: Clearly $\bigcup_{j=1}^{n} \Xi_{j} \subset \bigcap_{j=0}^{n}\left(A_{j} \cup A_{j}^{*}\right)$. Now, let $z \in \bigcap_{j=0}^{n}\left(A_{j} \cup A_{j}^{*}\right)$ and $k \in\{1, \cdots, n\}$ such that $z \in A_{j}, k \leqslant j \leqslant n$, and $z \in A_{j}^{*}, 1 \leqslant j \leqslant k-1$. It follows from Theorem 1.4.11 that $z \in A_{k} \cap A_{k-1}^{*}=$ $\Xi_{k}$. This completes the proof.

\subsubsection{Lyapunov functions and stability for gradient semigroups}

Inspired in the work of Conley (see [16, 44]) we state the equivalence between gradient semigroups and gradient-like semigroups relative to a disjoint family of isolated invariants $\Psi=\left\{\Xi_{1}, \cdots, \Xi_{n}\right\}$. The gradient-like semigroups relative to $\Psi$ were presented in Definition 
1.3.3 and now we present the gradient semigroups relative to $\Psi$.

Definition 1.4.13. We say that a semigroup $\{T(t): t \geqslant 0\}$ with a global attractor $\mathscr{A}$ and $a$ disjoint family of isolated invariants $\Psi=\left\{\Xi_{1}, \cdots, \Xi_{n}\right\}$ is a gradient semigroup relative to $\Psi$ if there exists a continuous function $V: X \rightarrow \mathbb{R}$ such that $[0, \infty) \ni t \mapsto V(T(t) x) \in \mathbb{R}$ is decreasing for each $x \in X, V$ is constant in $\Xi_{i}$ for each $1 \leqslant i \leqslant n$, and $V(T(t) x)=V(x)$ for all $t \geqslant 0$ if and only if $x \in \bigcup_{i=1}^{n} \Xi_{i}$. A function $V$ with such properties is called a Lyapunov function for $\{T(t): t \geqslant 0\}$.

We have the following equivalence between generalized gradient semigroups and generalized gradient-like semigroups.

Theorem 1.4.14. Let $\{T(t): t \geqslant 0\}$ be a semigroup with a global attractor $\mathscr{A}$ and a disjoint family os isolated invariants $\Psi=\left\{\Xi_{1}, \cdots, \Xi_{n}\right\}$. Then $\{T(t): t \geqslant 0\}$ is a gradient semigroup relative to $\Psi$ if and only if it is a gradient-like semigroup relative to $\Psi$. Moreover, the Lyapunov function $V: X \rightarrow \mathbb{R}$ of a gradient-like semigroup relative to $\Psi$ can be chosen such that $V\left(\Xi_{m}\right)=$ $m-1, m=1, \cdots, n$.

The equivalence between gradient semigroups and gradient-like semigroups relative to a disjoint family of isolated invariants along with Theorem 1.3.14, prove that the gradient semigroups relative to a disjoint family of isolated invariants are stable under certain perturbations; that is:

Theorem 1.4.15. Let $\left\{T_{\eta}(t): t \in \mathbb{T}^{+}\right\}, \eta \in[0,1]$, be a continuous and collective asymptotically compact family of semigroups at $\eta=0$. Assume that

(a) $\left\{T_{\eta}(t): t \geqslant 0\right\}$ has a global attractor $\mathscr{A}_{\eta}$ for each $\eta \in[0,1]$ and $\cup_{\eta \in[0,1]} \mathscr{A}_{\eta}$ is bounded.

(b) There exists $n \in \mathbb{N}$ such that $\mathscr{A}_{\eta}$ has $n$ isolated invariant sets $\Xi_{\eta}=\left\{\Xi_{1, \eta}^{*}, \cdots, \Xi_{n, \eta}^{*}\right\}$ for all $\eta \in[0,1]$ and $\sup _{1 \leqslant i \leqslant n}\left[\mathrm{~d}_{H}\left(\Xi_{i, \eta}^{*}, \Xi_{i, 0}^{*}\right)+\mathrm{d}_{H}\left(\Xi_{i, 0}^{*}, \Xi_{i, \eta}^{*}\right)\right] \stackrel{\eta \rightarrow 0}{\longrightarrow} 0$.

(c) $\left\{T_{0}(t): t \geqslant 0\right\}$ is a gradient semigroup relative to $\Xi_{0}$.

Then there exists $\eta_{0}>0$ such that, for all $\eta \leqslant \eta_{0},\left\{T_{\eta}(t): t \in \mathbb{T}^{+}\right\}$is a gradient semigroup relative to $\Xi_{\eta}$. 


\subsection{Exponential global attractors}

In this section, we will consider a class of semigroups $\{T(t): t \geqslant 0\}$ with a global attractor $\mathscr{A}$ and a disjoint family of isolated invariants $\Xi=\left\{\Xi_{1}, \cdots, \Xi_{n}\right\}$. If the unstable sets of these isolated invariants have the property of exponential local attraction and a Lipschitz condition (uniform in bounded sets and exponential growth in $t$ ), then the global attractor $\mathscr{A}$ exponentially attracts bounded sets of $X$. The results presented here extend the results in [2] for gradient semigroups and they can be found in [9].

In [2] the authors consider a $C^{1+\mu}$ gradient semigroup with global attractor $\mathscr{A}$ for which the set of equilibrium points $\mathscr{E}$ is composed only by hyperbolic equilibrium points (therefore, a finite set). For this semigroup, they prove that $\mathscr{A}$ attracts compact subsets exponentially (see Section 5.7 and Remark 7.11 in [2]).

In what follows, we extend the results of [2] in a broad perspective since we do not assume that the isolated invariant are equilibrium points and not even assume hyperbolicity.

\subsubsection{Gradient-like semigroups with exponential global attractors}

The next result is a simple consequence of the definition of gradient semigroups relative to a disjoint family of isolated invariants were firstly proven in [9].

Lemma 1.5.1. Let $\left\{T(t): t \in \mathbb{T}^{+}\right\}$be a gradient semigroup relative to the disjoint family of isolated invariants $\Xi=\left\{\Xi_{1}, \cdots, \Xi_{n}\right\}$. If $V: X \rightarrow \mathbb{R}$ is the associated Lyapunov function, $\Xi \in \Xi$ and $V(\Xi)$ is not a minimum value for $V$ in any neighborhood of $\Xi$, then there exists a global solution $\xi: \mathbb{T} \rightarrow X$ such that $\xi(\mathbb{T}) \not \subset \Xi$ and $\xi(t) \in W^{u}(\Xi)$ for all $t \in \mathbb{T}$.

The two following lemmas are natural extensions of Lemmas 1.3 .7 and 1.3 .8 for gradientlike semigroups relative to a disjoint family of isolated invariants.

Lemma 1.5.2. Let $\left\{T(t): t \in \mathbb{T}^{+}\right\}$be a gradient-like semigroup relative to a disjoint family of isolated invariants $\Psi=\left\{\Xi_{1}, \cdots, \Xi_{n}\right\}$.

Given $\Xi \in \Psi$ and $\epsilon>0$, there exists $\delta>0$ such that, if $v \in \mathscr{O}_{\delta}(\Xi)$ and for some $t_{1}>0, T\left(t_{1}\right) v \notin$ $\mathscr{O}_{\epsilon}(\Xi)$, then $T(t) v \notin \mathscr{O}_{\delta}(\Xi)$ for all $t \geqslant t_{1}$.

Proof: Assume that there exist an $\epsilon>0$, a sequence $\left\{v_{k}\right\}$ in $X$ with $v_{k} \stackrel{n \rightarrow \infty}{\longrightarrow} \Xi$, sequences $\left\{\tau_{k}\right\}$ and $\left\{t_{k}\right\}$ in $\mathbb{T}^{+}$with $\tau_{k}>t_{k}>0$, $\operatorname{dist}\left(T\left(\tau_{k}\right) v_{k}, \Xi\right) \stackrel{k \rightarrow \infty}{\longrightarrow} 0$ and $\operatorname{dist}\left(T\left(t_{k}\right) v_{k}, \Xi\right) \geqslant \epsilon$. Then $\Xi$ is 
chain recurrent in $\Psi$, which is a contradiction.

Lemma 1.5.3. Let $\left\{T(t): t \in \mathbb{T}^{+}\right\}$be a gradient-like semigroup relative to a disjoint family of isolated invariants $\Psi=\left\{\Xi_{1}, \cdots, \Xi_{n}\right\}$. If $B$ is a bounded subset of $X$, given $\epsilon>0$ there exists $t_{0} \in \mathbb{T}^{+}$such that

$$
\left\{T(t) x: t \in 0 \leqslant t \leqslant t_{0}\right\} \cap \mathscr{O}_{\epsilon}\left(\bigcup_{i=1}^{n} \Xi\right) \neq \varnothing, \quad \text { for all } x \in B
$$

Proof: Assume that there exist sequences $\left\{x_{k}\right\} \subset B, t_{k} \stackrel{k \rightarrow \infty}{\longrightarrow} \infty$ such that $\left\{T(s) x_{k}: 0 \leqslant s \leqslant\right.$ $\left.t_{k}\right\} \cap \mathscr{O}_{\epsilon}\left(\bigcup_{i=1}^{n} \Xi\right)=\varnothing$.

Choose $\tau_{k} \doteq$ the largest integer less than or equal $\frac{t_{k}}{2}$. Then there exists a subsequence of $\left\{T\left(\tau_{k}\right) x_{k}\right\}$ (which we will denote the same way) convergent to a certain $x_{0} \in X$. It is easy to see that $\left\{T(t) x_{0}: t \in \mathbb{T}^{+}\right\} \cap \mathscr{O}_{\epsilon}\left(\bigcup_{i=1}^{n} \Xi\right)=\varnothing$ and this contradicts the fact that $\bigcup_{i=1}^{n} \Xi$ attracts points.

Definition 1.5.4. Let $\left\{T(t): t \in \mathbb{T}^{+}\right\}$be a gradient-like semigroup relative to the disjoint family of isolated invariants $\Xi=\left\{\Xi_{1}, \cdots, \Xi_{n}\right\}$. We say that a local unstable set of $\Xi \in \Psi$ is exponentially attracting if there exist positive constants $C_{0}, \varrho_{0}$ and $\delta_{0}$ such that

$$
\operatorname{dist}\left(T(t) u_{0}, W_{\mathrm{loc}}^{u}(\Xi)\right) \leqslant C_{0} e^{-\varrho_{0} t}
$$

when $u_{0} \in \mathscr{O}_{\delta_{0}}(\Xi), t \in \mathbb{T}^{+}$and $\left\{T(s) u_{0}: 0 \leqslant s \leqslant t\right\} \subset \mathscr{O}_{\delta_{0}}(\Xi)$.

Theorem 1.5.5. Let $\left\{T(t): t \in \mathbb{T}^{+}\right\}$be a gradient-like semigroup relative to the disjoint family of isolated invariants $\Psi=\left\{\Xi_{1}, \ldots, \Xi_{p}\right\}$ and with a global attractor $\mathscr{A}$. Let $V$ be a closed, bounded and positively invariant neighborhood of $\mathscr{A}$ and assume that the restriction $\left.T(t)\right|_{V}$ of $T(t)$ to $V$ is a Lipschitz continuous function with Lipschitz constant ce ${ }^{L t}(L>0)$, for each $t \in \mathbb{T}^{+}$. Assume that each set $\Xi \in \Psi$ has an exponentially attracting unstable set.

Then, there exist a constant $\tilde{\gamma}>0$ and, for each $v \in V$, a function $\mathbb{T}^{+} \ni t \mapsto \tilde{u}(t) \in \mathscr{A}$ and $a$ constant $\tilde{c}>0$, such that

$$
d(T(t) \nu, \tilde{u}(t)) \leqslant \tilde{c} e^{-\tilde{\gamma} t}, t \in \mathbb{T}^{+} .
$$


Furthermore, the constant $\tilde{c}$ can be chosen independently of $v$ in bounded sets of $X$ and, consequently, for each $B \subset X$ bounded there exists a $c(B)>0$ such that

$$
\mathrm{d}_{H}(T(t) B, \mathscr{A}) \leqslant c(B) e^{-\tilde{\gamma} t}, t \in \mathbb{T}^{+} .
$$

Proof: We know that

$$
d\left(T(t) w_{1}, T(t) w_{2}\right) \leqslant c e^{L t} d\left(w_{1}, w_{2}\right), \quad \forall w_{1}, w_{2} \in V, t \in \mathbb{T}^{+}
$$

Choose $\delta, \gamma>0$ and $c>0$, such that

$$
d\left(T(t) w, W_{l o c}^{u}\left(\Xi_{j}\right)\right) \leqslant c e^{-\gamma t} \text { for all } j=1, \ldots, p,
$$

when $t \in \mathbb{T}^{+} \mathrm{e}\{T(s) w: 0 \leqslant s \leqslant t\} \subset \mathscr{O}_{\delta}\left(\Xi_{j}\right)$.

From Lemma 1.5.2, choose $\delta^{\prime}<\delta$ such that, if $v \in \mathscr{O}_{\delta^{\prime}}\left(\Xi_{j}\right)$, and for some $t_{1} \in \mathbb{T}^{+}$,

$$
T\left(t_{1}\right) v \notin \mathscr{O}_{\delta}\left(\Xi_{j}\right)
$$

then

$$
T(t) v \notin \mathscr{O}_{\delta^{\prime}}\left(\Xi_{j}\right) \text {, for all } t \geqslant t_{1} \text {. }
$$

Now, from Lemma 1.5.3, there exists $t_{0} \in \mathbb{T}^{+}$such that, for all $v \in V$

$$
\left\{T(t) v: 0 \leqslant t \leqslant t_{0}\right\} \cap \bigcup_{j=1}^{p} \mathscr{O}_{\delta^{\prime}}\left(\Xi_{j}\right) \neq \varnothing .
$$

Therefore, given $v \in V$, there exist sequences $\left\{t_{i(j)}^{-}\right\}_{j=1}^{m},\left\{t_{i(j)}^{+}\right\}_{j=1}^{m}$ and $\left\{\Xi_{i(j)}\right\}_{j=1}^{m}$ such that $i(j) \in\{1, \ldots, p\}, 1 \leqslant j \leqslant m \leqslant p$,

$$
t_{i(1)}^{-} \leqslant t_{0}, \quad 0<t_{i(j)}^{-}-t_{i(j-1)}^{+} \leqslant t_{0}, 2 \leqslant j \leqslant m, \quad t_{i(m)}^{+}=+\infty
$$

$T(t) v \in \mathscr{O}_{\delta}\left(\Xi_{i(j)}\right)$ for all $t_{i(j)}^{-} \leqslant t<t_{i(j)}^{+}, T\left(t_{i(j)}^{+}\right) v \notin \mathscr{O}_{\delta}\left(\Xi_{i(j)}\right)$ and $j \in\{1, \ldots, m\}$.

Given $v \in V$, the positive orbit of $v$ visits neighborhoods of the invariant sets belonging to $\Psi$. We reorder and rename this sets $\Xi_{1}, \ldots, \Xi_{m}, m \leqslant p$, using the order in which the $\delta^{\prime}$ neighborhood of them is visited by the orbit of $v$. 
Now choose a point $y_{1} \in \Xi_{1}$ and for each $t_{j}^{-} \leqslant t \leqslant t_{j}^{+}$choose $\psi(t)$ such that

$$
\operatorname{dist}\left(T(t) v, \overline{W_{\mathrm{loc}}^{\mathrm{u}}\left(\Xi_{j}\right)}\right)=d(T(t) v, \psi(t)), \quad 1 \leqslant j \leqslant m
$$

Define $\tilde{u}: \mathbb{T}^{+} \rightarrow \mathscr{A}$ by

$$
\tilde{u}(t)=\left\{\begin{array}{l}
y_{1}, 0 \leqslant t<t_{1}^{-}, \\
\psi(t), t_{1}^{-} \leqslant t \leqslant t_{1}^{+}, \\
T\left(t-t_{j-1}^{+}\right) \tilde{u}\left(t_{j-1}^{+}\right), t_{j-1}^{+} \leqslant t \leqslant \kappa_{j}^{0}, 2 \leqslant j \leqslant n, \\
\psi(t), \kappa_{j}^{0}<t \leqslant t_{j}^{+}, 2 \leqslant j \leqslant n .
\end{array}\right.
$$

where $k_{j}^{0} \in\left(t_{j}^{-}, t_{j}^{+}\right]$is given by

$$
\gamma_{1}:=\gamma, \quad \gamma_{j}=\frac{\gamma_{j-1}^{2}}{L+2 \gamma_{j-1}} \quad \text { e } \quad \kappa_{j}^{0}=\min \left\{\frac{L+2 \gamma_{j-1}}{L+\gamma_{j-1}} t_{j}^{-}, t_{j}^{+}\right\}, \quad j=2, \ldots, m
$$

By hypothesis we have that

$$
\begin{aligned}
& d(T(t) \nu, \tilde{u}(t)) \leqslant \sup _{\nu \in V} d\left(\nu, y_{1}\right) e^{\gamma t_{0}} e^{-\gamma t} \doteq \tilde{c}_{1} e^{-\gamma t}, 0 \leqslant t<t_{1}^{-}, \\
& d(T(t) v, \tilde{u}(t)) \leqslant c e^{-\gamma\left(t-t_{1}^{-}\right)} \leqslant c e^{\gamma t_{0}} e^{-\gamma t} \doteq \hat{c}_{1} e^{-\gamma t}, t_{1}^{-} \leqslant t \leqslant t_{1}^{+} .
\end{aligned}
$$

Let $c_{1}=\max \left\{\tilde{c}_{1}, \hat{c}_{1}\right\}$. We show that, for each $j=2, \ldots, m$, the following statement is true

$$
\begin{aligned}
& \text { if (i) } d(T(t) v, \tilde{u}(t)) \leqslant c_{j-1} e^{-\gamma_{j-1} t}, t_{j-2}^{+} \leqslant t<t_{j-1}^{+} \text {with some } c_{j-1}>0 \text {, } \\
& \text { then(ii) } d(T(t) v, \tilde{u}(t)) \leqslant c_{j} e^{-\gamma_{j} t}, t_{j-1}^{+} \leqslant t<t_{j}^{+} \text {with some } c_{j}>0
\end{aligned}
$$

Firstly note that, by hypothesis, if $t_{j-1}^{+} \leqslant t \leqslant \kappa_{j}^{0}$,

$$
\begin{aligned}
d(T(t) v, \tilde{u}(t)) & \leqslant c e^{L\left(t-t_{j-1}^{+}\right)} d\left(T\left(t_{j-1}^{+}\right) v, \tilde{u}\left(t_{j-1}^{+}\right)\right) \\
& \stackrel{\text { (i) }}{\leqslant} c c_{j-1} e^{L\left(t-t_{j-1}^{+}\right)-\gamma_{j-1} t_{j-1}^{+}} \cdot \\
& \leqslant c c_{j-1} e^{(L+\gamma) t_{0}} e^{L\left(t-t_{j}^{-}\right)-\gamma_{j-1} t_{j}^{-}}
\end{aligned}
$$

and for $\kappa_{j}^{0} \leqslant t \leqslant t_{j}^{+}$,

$$
d(T(t) v, \psi(t)) \leqslant c e^{-\gamma\left(t-t_{j}^{-}\right)} \leqslant c e^{-\gamma_{j-1}\left(t-t_{j}^{-}\right)} .
$$


Taking a closer look at (1.5.7) and (1.5.8) we can note that, if $t_{j}^{-}<\kappa_{j}^{0}<t_{j}^{+}$, we have that 1

$$
L\left(\kappa_{j}^{0}-t_{j}^{-}\right)-\gamma_{j-1} t_{j}^{-}=-\gamma_{j-1}\left(\kappa_{j}^{0}-t_{j}^{-}\right)=-\gamma_{j} \kappa_{j}^{0}
$$

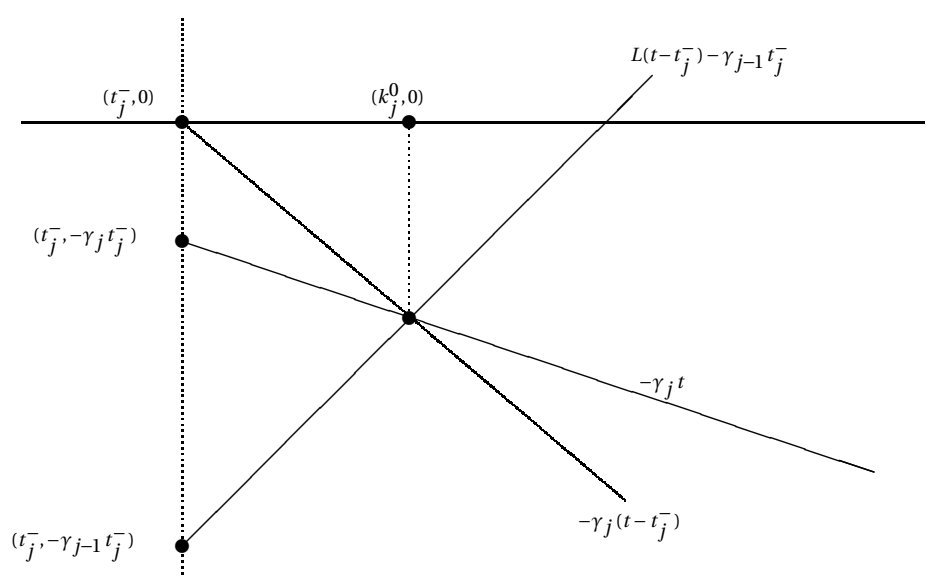

Figure: Determination of $k_{j}^{0}$ and $\gamma_{j}$.

Indeed, we claim that

$$
\begin{gathered}
L\left(t-t_{j}^{-}\right)-\gamma_{j-1} t_{j}^{-} \leqslant-\gamma_{j} t, \quad t_{j}^{-} \leqslant t \leqslant \kappa_{j}^{0} . \\
-\gamma_{j-1}\left(t-t_{j}^{-}\right) \leqslant-\gamma_{j} t, \quad \kappa_{j}^{0}<t \leqslant t_{j}^{+} .
\end{gathered}
$$

Now we are ready to complete the estimate. From (1.5.7) and (1.5.10) we obtain that, for $t_{j}^{-} \leqslant t \leqslant \kappa_{j}^{0}$

$$
d(T(t) v, \tilde{u}(t)) \leqslant c c_{j-1} e^{(L+\gamma) t_{0}} e^{-\gamma_{j} t}
$$

while (1.5.8) and (1.5.11) ensure that, for $\kappa_{j}^{0}<t \leqslant t_{j}^{+}$,

$$
d(T(t) v, \tilde{u}(t)) \leqslant c e^{-\gamma_{j-1}\left(t-t_{j}^{-}\right)} \leqslant c e^{-\gamma_{j} t}
$$

\footnotetext{
${ }^{1}$ The first equality in (1.5.9) determines $k_{j}^{0}$ in terms of $\gamma_{j-1}$ and $t_{j}^{-}$; and, once determined $k_{j}^{0}$, the second equality in (1.5.9) determines $\gamma_{j}$ in terms of $\gamma_{j-1}$. As a result we have the expressions presented in (1.5.6).
} 
From (1.5.7), for $t_{j-1}^{+} \leqslant t \leqslant t_{j}^{-}$,

$$
d(T(t) \nu, \tilde{u}(t)) \leqslant c c_{j-1} e^{L\left(t-t_{j-1}^{+}\right)-\gamma_{j-1}\left(t_{j-1}^{+}-t+t\right)} \leqslant c c_{j-1} e^{(L+\gamma) t_{0}} e^{-\gamma_{j} t}
$$

and (ii) holds with

$$
c_{j}=\max \left\{c, c c_{j-1} e^{(L+\gamma) t_{0}}\right\},
$$

and completes the proof. 


\section{Chapter}

\section{2}

\section{Evolution processes}

Let $X$ be a metric space and $\mathrm{d}(\cdot, \cdot): X \times X \rightarrow[0, \infty)$ its metric. Recall that $\mathbb{T}$ denotes $\mathbb{R}$ or $\mathbb{Z}\left(\mathbb{T}_{t}^{+}=\{s \in \mathbb{T}: s \geq t\}, \mathbb{T}_{t}^{-}=\{s \in \mathbb{T}: s \leq t\}\right), \mathscr{C}(X)$ denotes the set of continuous maps from $X$ into itself and $\mathscr{P}=\left\{(t, s) \in \mathbb{T}^{2}: t \geqslant s\right\}$. An evolution process in $X$ is a family of maps $\{S(t, s):(t, s) \in \mathscr{P}\}$ in $\mathscr{C}(X)$ with the following properties

1. $S(t, t)=I$, for all $t \in \mathbb{T}$,

2. $S(t, s)=S(t, \tau) S(\tau, s)$, for all $t \geqslant \tau \geqslant s$,

3. $\mathscr{P} \times X \ni(t, s, x) \mapsto S(t, s) x \in X$ is continuous.

If $X$ is a normed vector space and $S(t, s)$ is linear for each $(t, s) \in \mathscr{P}$ we say that $\{S(t, s): t \geqslant$ $s \in \mathbb{T}\}$ is a linear evolution process. This chapter is done following basically [6] and the reader can also check [7] for more details.

\subsection{Pullback attractors}

In this section we present the notion of pullback attractors, the notions necessary to present this concept and its relation to the global attractors of semigroups. For an evolution process $\{S(t, s):(t, s) \in \mathscr{P}\}$ and a subset $B \subset X$, we define:

(a) For each $(t, s) \in \mathscr{P}$, the image of $B$ under $S(t, s)$,

$$
S(t, s) B \doteq\{S(t, s) b: b \in B\}
$$


(b) The orbit of $B$ starting at time $s \in \mathbb{T}$

$$
\gamma^{s}(B) \doteq \bigcup_{t \geqslant s} S(t, s) B
$$

(c) The pullback orbit of $B$ at time $t \in \mathbb{T}$,

$$
\gamma_{p}(B, t) \doteq \bigcup_{s \leqslant t} S(t, s) B
$$

Definition 2.1.1. Let $\{S(t, s):(t, s) \in \mathscr{P}\}$ be an evolution process. Given $t \in \mathbb{T}$, we say that a set $B(t) \subset X$ pullback attracts (pullback absorbs) bounded subsets of $X$ at time $t$ under the action of $\{S(t, s):(t, s) \in \mathscr{P}\}$ if

$$
\lim _{s \rightarrow-\infty} \operatorname{dist}_{H}(S(t, s) D, B(t))=0 \quad(\exists T=T(t, D) \leqslant t \text { such that } S(t, s) D \subset B(t), \forall s \leqslant T) .
$$

for each bounded subset $D$ of $X$. A family $\{B(t): t \in \mathbb{T}\}$ pullback attracts (pullback absorbs) bounded subsets of $X$ under the action of $\{S(t, s):(t, s) \in \mathscr{P}\}$ if $B(t)$ pullback attracts (pullback absorbs) bounded subsets of $X$ at time $t$ under the action of $\{S(t, s):(t, s) \in \mathscr{P}\}$, for each $t \in \mathbb{T}$.

If the exists a family $\{B(t): t \in \mathbb{R}\}$ of bounded subsets which pullback attracts (pullback absorbs) bounded subsets of $X$ we say that $\{S(t, s):(t, s) \in \mathscr{P}\}$ is pullback bounded dissipative.

Remark 2.1.2. In this definition, the final time is fixed while the initial time goes to $-\infty$. Note that this is not the same that go back in time. The evolution is always until the future instant $t$ starting at an initial time $s$ going to $-\infty$.

Note that, if a set pullback absorbs bounded sets at time $t$ then it pullback attracts bounded sets at time $t$.

Definition 2.1.3. Let $\{B(t): t \in \mathbb{R}\}$ a family of subsets of $X$. We say that this family is invariant under the evolution process $\{S(t, s):(t, s) \in \mathscr{P}\}$ if

$$
S(t, s) B(s)=B(t), \text { for all }(t, s) \in \mathscr{P} \text {. }
$$


Definition 2.1.4. Let $\{S(t, s):(t, s) \in \mathscr{P}\}$ be an evolution process in a metric space $X$. We say that a family $\{\mathscr{A}(t): t \in \mathbb{R}\}$ of compact subsets of $X$ is a pullback attractor for $\{S(t, s):(t, s) \in \mathscr{P}\}$ if it is invariant, pullback attracts bounded sets of $X$ and is the family of closed sets which is minimal with the property of pullback bounded dissipation; that is, if another family $\{C(t)$ : $t \in \mathbb{R}\}$ of closed sets pullback attracts bounded subsets of $X$, then $A(t) \subseteq C(t)$, for all $t \in \mathbb{R}$.

\section{Remark 2.1.5.}

1. We remark that the requirement of minimality in Definition 2.1.4 is additional relative to the theory of global attractors for semigroups. This requirement is essential to ensure the uniqueness of pullback attractors. The addition of the requirement is related to the weakening of the invariance property imposed by the non-autonomous nature of the evolution processes along with the possibility of the pullback attractor being unbounded at $-\infty$; that is, we allow that $\cup_{s \leqslant t} \mathscr{A}(s)$ is unbounded for all $t \in \mathbb{R}$. If $\{T(t): t \geqslant 0\}$ and $\{T(t-s):(t, s) \in \mathscr{P}\}$ is the associated non-autonomous evolution process, there might be a family $\{\mathscr{A}(t): t \in \mathbb{T}\}$ of compact invariant sets which pullback attracts bounded subsets of $X$ and it is not minimal. In fact, if $T(t-s)=e^{-(t-s)} x_{0}, x_{0} \in \mathbb{T},(t, s) \in \mathscr{P}$, the family $\left\{\left[-e^{-t}, e^{-t}\right]: t \in \mathbb{T}\right\}$ is invariant, $\left[-e^{-t}, e^{-t}\right]$ is compact and pullback attracts bounded subsets of $\mathbb{T}$ at time $t$ for each $t \in \mathbb{T}$.

2. The requirement of minimality in Definition 2.1 .4 may be eliminated if we ask that $\cup_{s \leqslant t} \mathscr{A}(s)$ is bounded for all $t \in \mathbb{T}$.

Definition 2.1.6. We say that a solution $\xi: \mathbb{T} \rightarrow X$ of an evolution process $\{S(t, s):(t, s) \in \mathscr{P}\}$ is bounded backwards if there exists $\tau \in \mathbb{T}$ such that the $\operatorname{set}\{\xi(t): t \leqslant \tau\}$ is bounded.

\section{Remark 2.1.7.}

1. It is not hard to see that if an evolution process $\{S(t, s):(t, s) \in \mathscr{P}\}$ has a pullback attractor $\{\mathscr{A}(t): t \in\}$ and $\xi: \mathbb{T} \rightarrow X$ is a bounded backwards solutions, then $\xi(t) \in \mathscr{A}(t)$ for all $t \in \mathbb{T}$.

2. Also, if $\{\mathscr{A}(t): t \in \mathbb{T}\}$ is a pullback attractor for the evolution process $\{S(t, s):(t, s) \in \mathscr{P}\}$ 
and $\bigcup_{s \leqslant t} \mathscr{A}(s)$ is bounded for all $t \in \mathbb{R}$, then $\mathscr{A}(t)$ is given by

$$
\begin{aligned}
\mathscr{A}(t)=\{\xi(t) \mid \xi: \mathbb{T} \rightarrow X \text { is a global solution } \\
\text { bounded backwards for }\{S(t, s):(t, s) \in \mathscr{P}\}\}, \forall t \in \mathbb{T} .
\end{aligned}
$$

The next result relates the pullback attractors of autonomous evolution processes and global attractors for semigroups. This result shows that the concept of pullback attractor extends, in a natural way, the concept of global attractors for semigroups to the evolution processes.

Theorem 2.1.8. If $\{T(t): t \geqslant 0\}$ is a semigroup and $S(t, s)=T(t-s),(t, s) \in \mathscr{P}$ is the associated evolution process then $\{T(t): t \geqslant 0\}$ has a global attractor $\mathscr{A}$ if and only if $\{S(t, s):(t, s) \in \mathscr{P}\}$ has a pullback attractor $\{\mathscr{A}(t): t \in \mathbb{T}\}$. In any one of the cases $\mathscr{A}(t)=\mathscr{A}$ for all $t \in \mathbb{T}$.

\subsection{Existence of pullback attractors}

As in the autonomous case, the notion of $\omega$-limit will play an important role in the theory of pullback attractors for evolution processes. Recall that $\mathbb{T}_{t}^{-}=\{s \in \mathbb{T}, s \leqslant t\}$.

Definition 2.2.1. Let $\{S(t, s):(t, s) \in \mathscr{P}\}$ be an evolution process in a metric space $X$ and $B$ a subset of $X$. The pullback $\omega$-limit of $B$ is defined by

$$
\omega(B, t) \doteq \bigcap_{\sigma \leqslant t} \overline{\bigcup_{s \leqslant \sigma} S(t, s) B}
$$

For each subset $B$ of $X$, we have that

$$
\begin{array}{r}
\omega(B, t)=\left\{y \in X: \text { there exist sequences }\left\{s_{k}\right\}_{k \in \mathbb{N}} \text { in } \mathbb{T}_{t}^{-}, s_{k} \stackrel{k \rightarrow \infty}{\longrightarrow}-\infty\right. \\
\text { and } \left.\left\{x_{k}\right\}_{k \in \mathbb{N}} \text { in } B, \text { such that } y=\lim _{k \rightarrow \infty} S\left(t, s_{k}\right) x_{k}\right\} .
\end{array}
$$

Clearly, if $\{S(t): t \geqslant 0\}$ is a semigroup and $S(t, s)=S(t-s),(t, s) \in \mathscr{P}$ we have that $\omega(B, t) \doteq$ $\cap_{s \geqslant 0} \overline{\bigcup_{r \geqslant s} S(r) B}$ is independent of $t$ and coincides with the definition of the $\omega$-limit $\omega(B)$ of $B$ for semigroups (see [17, 46, 25]).

The result about the existence of pullback attractors is a generalization of an analogous result to autonomous processes (see [2, 17, 25, 42, 46]): 
Theorem 2.2.2. Let $\{S(t, s):(t, s) \in \mathscr{P}\}$ be an evolution process in a metric space $X$. Then, the following statements are equivalent

(a) $\{S(t, s):(t, s) \in \mathscr{P}\}$ has a pullback attractor $\{\mathscr{A}(t): t \in \mathbb{T}\}$.

(b) There exists a family of compact sets $\{K(t): t \in \mathbb{T}\}$ which pullback attracts bounded subsets of $X$ under the action of $\{S(t, s):(t, s) \in \mathscr{P}\}$.

In any of the cases

$$
\mathscr{A}(t)=\overline{\bigcup\{\omega(B, t): B \subset X, B \text { bounded }\}}
$$

\subsection{Gradient-like evolution processes}

\subsubsection{Definition and main properties}

In this section we extend the concept of gradient-like semigroups to evolution processes. Note that a process will not, in general, possess any equilibrium point. Hence, a generalization of the concept of gradient-like semigroups to processes will require a structure that replaces the role that an equilibrium has for a gradient-like semigroup. Those structures are the isolated invariant families which we define next inspired by the definition of isolated invariant sets given in Definition 1.3.1.

Definition 2.3.1. Let $\Xi \doteq\{\Xi(t): t \in \mathbb{T}\}$ be an invariant family for the evolution process $\{S(t, s)$ : $(t, s) \in \mathscr{P}\}$. The family $\Xi$ is called isolated if there exists $a \delta>0$ such that any global solution $\xi(\cdot)$ with $\xi(t) \in \mathscr{O}_{\delta}(\Xi(t))$ for all $t \in \mathbb{T}$ must be in $\Xi(\cdot)$, i.e. $\xi(t) \in \Xi(t)$ for all $t \in \mathbb{T}$. A collection $\Psi=$ $\left\{\Xi_{1}, \cdots \Xi_{\mathfrak{p}}\right\}$ of isolated invariant sets is said a disjoint collection of isolated invariant families if each $\Xi_{i}^{*}$ is an isolated invariant family and there exists a $\delta^{*}>0$ such that for every $t \in \mathbb{R}$

$$
\mathscr{O}_{\delta^{*}}\left(\Xi_{i}(t)\right) \cap \mathscr{O}_{\delta^{*}}\left(\Xi_{j}(t)\right)=\varnothing \quad \text { for all } \quad i \neq j
$$

Remark 2.3.2. Let $X$ be a Banach space, $\{S(t): t \geqslant 0\}$ be a nonlinear semigroup on $X,\{S(t, s)$ : $\left.S(t, s)=S(t-s),(t, s) \in \mathscr{P}_{\mathbb{R}}\right\}$ be the associated nonlinear evolution process. If $J$ is an isolated invariant set for $\{S(t): t \geqslant 0\}$, then $\mathscr{J}=\{J(t) \subset X: J(t)=J, t \in \mathbb{R}\}$ is an isolated invariant family for $\left\{S(t, s):(t, s) \in \mathscr{P}_{\mathbb{R}}\right\}$. 
Definition 2.3.3. Let $\left\{S(t, s):(t, s) \in \mathscr{P}_{\mathbb{R}}\right\}$ be an evolution process and let $\Psi=\left\{\Xi_{1}, \cdots \Xi_{\mathfrak{p}}\right\}$ be a disjoint collection of isolated invariant families in the pullback attractor $\{\mathscr{A}(t): t \in \mathbb{R}\} . A$ homoclinic structure in $\Psi$ is a non-trivial set of orbits between elements of $\Psi$ that form a cycle: a nonempty set of global solutions $\left\{\xi_{i}: \mathbb{R} \rightarrow X\right\}_{i=1}^{k}$ such that

$$
\lim _{t \rightarrow-\infty} \operatorname{dist}\left(\xi_{i}(t), \Xi_{\ell_{i}}(t)\right)=0 \quad \text { and } \quad \lim _{t \rightarrow+\infty} \operatorname{dist}\left(\xi_{i}(t), \Xi_{\ell_{i+1}}\right)=0, \quad 1 \leq i \leq k,
$$

where $\Xi_{\ell_{i}}(\cdot) \in \Psi$ and $\Xi_{\ell_{k+1}}(\cdot)=\Xi_{\ell_{1}}(\cdot)$.

Definition 2.3.4. Let $X$ be a Banach space and $\left\{S(t, s):(t, s) \in \mathscr{P}_{\mathbb{R}}\right\}$ be an evolution process in $X$ which has a pullback attractor $\{\mathscr{A}(t): t \in \mathbb{R}\}$. We say that $\left\{S(t, s):(t, s) \in \mathscr{P}_{\mathbb{R}}\right\}$ is a generalized gradient-like process if the following two hypotheses are satisfied:

(H1) There is a finite set $\Psi=\left\{\Xi_{i}: \mathbb{R} \rightarrow X: 1 \leqslant i \leqslant \mathfrak{p}\right\}$ of isolated invariant families in $\{\mathscr{A}(t)$ : $t \in \mathbb{R}\}$ with the property that any global solution $\xi: \mathbb{R} \rightarrow X$ in $\{\mathscr{A}(t): t \in \mathbb{R}\}$ satisfies

$$
\lim _{t \rightarrow-\infty} \operatorname{dist}\left(\xi(t), \Xi_{i}(t)\right)=0 \text { and } \lim _{t \rightarrow \infty} \operatorname{dist}\left(\xi(t), \Xi_{j}(t)\right)=0
$$

for some $1 \leqslant i, j \leqslant \mathfrak{p}$.

(H2) $\Psi=\left\{\Xi_{1}, \cdots, \Xi_{\mathfrak{p}}\right\}$ does not contain any homoclinic structure.

When $\left\{S(t, s)=S(t-s):(t, s) \in \mathscr{P}_{\mathbb{R}}\right\}$ we say that $\{S(t): t \geqslant 0\}$ is a generalized gradient-like semigroup.

Next we introduce the definitions of unstable and stable sets:

Definition 2.3.5. Let $\left\{S(t, s):(t, s) \in \mathscr{P}_{\mathbb{R}}\right\}$ be an evolution process. The unstable set of an isolated invariant family $\Xi$ is the set

$$
\begin{gathered}
W^{\mathrm{u}}\left(\Xi^{*}\right)=\{(\tau, \zeta) \in \mathbb{R} \times X: \text { there exists a global solution } \xi: \mathbb{R} \rightarrow X \\
\text { such that } \left.\xi(\tau)=\zeta \text { and } \lim _{t \rightarrow-\infty} \operatorname{dist}(\xi(t), \Xi(t))=0\right\} .
\end{gathered}
$$

Also, $W^{\mathrm{u}}\left(\Xi^{*}\right)(\tau) \doteq\left\{\zeta \in X:(\tau, \zeta) \in W^{\mathrm{u}}\left(\Xi^{*}\right)\right\}$. 
Remark 2.3.6. We note that when the evolution process $\{S(t, s): s \geqslant t \in \mathbb{R}\}$ comes from a nonlinear semigroup $\{S(t): t \geqslant 0\}\left(S(t, s)=S(t-s)\right.$, for all $\left.(t, s) \in \mathscr{P}_{\mathbb{R}}\right)$, the above definition of stable e unstable set coincide with the usual definition of a unstable set of an invariant set.

Unfortunately, in the case of a general evolution process $\left\{S(t, s):(t, s) \in \mathscr{P}_{\mathbb{R}}\right\}$ this may not hold. To make the usual definition of unstable sets for an invariant family $\{\Xi(t): t \in \mathbb{R}\}$ coincide with Definition 2.3.5 we ask the following additional condition

If a solution $\xi(t)$ stays inside a suitably small neighborhood of $\Gamma_{i}^{*}$ for all $t$ in an interval of the form $\left(-\infty, t_{0}\right]$ (respectively, $\left[t_{0}, \infty\right)$ ), then $\operatorname{dist}(\xi(t), \Xi(t)) \stackrel{t \rightarrow-\infty}{\longrightarrow} 0$ (respectively, $\operatorname{dist}(\xi(t), \Xi(t)) \stackrel{t \rightarrow \infty}{\longrightarrow} 0)$.

This condition is automatically satisfied in the case when the evolution process is given by a semigroup, as seen in the next lemma.

Lemma 2.3.7. Let $\{S(t): t \geqslant 0\}$ be an asymptotically compact semigroup, $\Xi \subset X$ be an isolated invariant set and $\xi: \mathbb{R} \rightarrow X$ be a global solution for $\{S(t): t \geqslant 0\}$. Assume that $U, V$ are open subsets of $X$ with $\bar{U} \subset V$ e such that $\Xi \subset U, \Xi$ being the maximal invariant set contained in $V$. If there is a $t_{0} \in \mathbb{R}$ such that $\xi(t) \in U$ for all $t \geqslant t_{0}$ (respectively, $t \leqslant t_{0}$ ), then $\xi(t) \stackrel{t \rightarrow \infty}{\longrightarrow} \Xi$ (respectively, $\xi(t) \stackrel{t \rightarrow-\infty}{\longrightarrow} \Xi$ ).

Proof: Let us prove the case $\xi(t) \in U$ for all $t \geqslant t_{0}$. The other case is completely similar. We argue by contradiction assuming that

$$
\limsup _{t \rightarrow \infty} \operatorname{dist}(\xi(t), \Xi)>0
$$

Then, there exist $\epsilon>0$ and a sequence $t_{k} \rightarrow \infty$ such that $\operatorname{dist}\left(\xi\left(t_{k}\right), \Xi\right) \geqslant \epsilon$. From the asymptotic compactness of the semigroup, this sequence has a convergent subsequence (which we denote the same). If $y=\lim _{k \rightarrow \infty} \xi\left(t_{k}\right)$, then $\operatorname{dist}(y, \Xi) \geqslant \epsilon$ and $y$ belongs to the set $\omega$-limit set of $x_{0}=\xi(0)$. Thus $y \in \omega\left(x_{0}\right) \subset \bar{U}$ and $\omega\left(x_{0}\right)$ is invariant under $\{S(t): t \geqslant 0\}$. That contradicts the assumption that $\Xi$ is the maximal invariant subset of $V$ and concludes the proof.

\subsubsection{Gradient-like processes under perturbation}

We are now ready to state the main result of this section 
Theorem 2.3.8. Let $X$ be a Banach space, $\eta \in[0,1]$ be a parameter and $\left\{S_{\eta}(t, s):(t, s) \in \mathscr{P}\right\}$ be a nonlinear evolution process in $X$ with a pullback attractor $\left\{\mathscr{A}_{\eta}(t): t \in \mathbb{T}\right\}$. Assume that,

(a) $\overline{\cup_{\eta \in[0,1]} \cup_{t \in \mathbb{R}} \mathscr{A}_{\eta}(t)}$ is compact.

(b) $S_{0}(t, s)=S(t-s), t \geqslant s$ and $\{S(t): t \geqslant 0\}$ is a generalized gradient-like nonlinear semigroup with isolated invariant sets $\left\{\Xi_{1,0}^{*}, \cdots \Xi_{n, 0}^{*}\right\}$.

(c) $\left\{S_{\eta}(t, s): t \geqslant s \in \mathbb{R}\right\}$ has finitely many isolated invariant families $\Psi_{\eta}=\left\{\Xi_{1, \eta}^{*}(\cdot), \cdots, \Xi_{n, \eta}^{*}(\cdot)\right\}$ with

$$
\lim _{\eta \rightarrow 0}\left[\sup _{t \in \mathbb{R}} \operatorname{dist}_{H}\left(\Xi_{i, \eta}^{*}(t), \Xi_{i, 0}^{*}(t)\right)\right]=0, \text { for each } 1 \leqslant i \leqslant n
$$

(d) $\left\|S_{\eta}(t+\tau, \tau) u-S_{0}(t+\tau, \tau) u\right\|_{X} \stackrel{\eta \rightarrow 0}{\longrightarrow} 0$ uniformly for $\tau \in \mathbb{R}$ and for $(t, u)$ in compact subsets of $\mathbb{T}^{+} \times X$.

(e) There exists $\delta>0$ and $\eta_{1}>0$ such that for all $\eta \in\left(0, \eta_{1}\right)$, if $\xi_{\eta}$ is a global solution in $\left\{\mathscr{A}_{\eta}(t): t \in \mathbb{T}\right\}$ such that if $\limsup _{t \rightarrow \pm \infty} \operatorname{dist}\left(\xi_{\eta}(t), \Xi_{i, \eta}^{*}(t)\right)<\delta$ then

$$
\operatorname{dist}\left(\xi_{\eta}(t), \Xi_{i, \eta}^{*}(t)\right) \rightarrow 0 \text { as } t \rightarrow \pm \infty \text {. }
$$

Then there exists $\eta_{0}>0$ such that, for all $\eta \leqslant \eta_{0},\left\{S_{\eta}(t, s):(t, s) \in \mathscr{P}_{\mathbb{R}}\right\}$ is a generalized gradientlike nonlinear evolution process. Consequently, there exists $\eta_{0}>0$ such that

$$
\mathscr{A}_{\eta}(t)=\cup_{i=1}^{n} W^{u}\left(\Xi_{i, \eta}^{*}\right)(t), \text { for all } t \in \mathbb{R} \text { and } \eta \in\left[0, \eta_{0}\right]
$$

Theorem 2.3 .8 generalizes the characterization result in [8] to perturbation of autonomous generalized gradient-like nonlinear semigroups. Hence, the limit problem does not need to have a Lyapunov function, and $\Xi_{i}^{*}$ does not need to be an equilibrium point. Of course it remains to prove in applications the continuity of the isolated global solutions $\Xi_{i, \eta}^{*}$ at $\eta=0$, which is known to hold for example for normally hyperbolic global solutions.

Observe that Theorem 2.3.8 implies that the pullback attractors for $\left\{S_{\eta}(t, s):(t, s) \in \mathscr{P}_{\mathbb{R}}\right\}$ may be, in some situations, characterized as the union of the unstable sets of isolated global solutions. Hyperbolicity is not required up to this point. Nonetheless, the persistence of the isolated global solutions will require some kind of hyperbolicity (in general normal hyperbolicity). 


\section{Chapter}

\section{An estimate on the fractal dimension}

We know begin the main part of this work, and use the structure of the global attractor to estimate its fractal dimension. Before we proceed, let us briefly recall the definitions of topological, Hausdorff and fractal dimension.

If $K$ is a topological space, we say that $K$ has finite topological dimension if there exists a natural number $d$ such that, for every open covering $\mathscr{U}$ of $K$, there is another covering $\mathscr{U}^{\prime}$ of $K$ refining $\mathscr{U}$ with the property that each point of $K$ belongs to at most $d+1$ sets in $\mathscr{U}^{\prime}$. In this case, the topological dimension $\operatorname{dim}_{T}(K)$ of $K$ is the minimum $d$ with this property. With this notion, a subset of $\mathbb{R}^{n}$ with non-empty interior has topological dimension $n$ and, if $K$ is a compact metric space with topological dimension $\operatorname{dim}_{T}(K)<\infty$, then it is homeomorphic to a subset of $\mathbb{R}^{n}$ with $n=2 \operatorname{dim}_{T}(K)+1$ (see [33], [41]). For a more detailed discussion, the reader can see [6].

Next we introduce the notion of Hausdorff dimension. For a given metric space $(X, \rho)$, $\alpha>0, \epsilon>0$ and $A \subset X$ let

$$
\mu_{\epsilon}^{(\alpha)}(A)=\inf \left\{\sum_{i=1}^{\infty}\left(\operatorname{diam}\left(B_{i}\right)\right)^{\alpha}: A \subset \cup_{i=1}^{\infty} B_{i}, \operatorname{diam}\left(B_{i}\right)<\epsilon\right\},
$$

with the convention inf $\varnothing=\infty$. Since $\mu_{\epsilon}^{(\alpha)}(A)$ increases as $\epsilon$ decreases, we define

$$
\mu^{(\alpha)}(A)=\lim _{\epsilon \rightarrow 0} \mu_{\epsilon}^{(\alpha)}(A)
$$

We have the following property of the set function $\mu^{(\alpha)}: 2^{X} \rightarrow[0, \infty]$ (where $2^{X}=\{A: A \subset$ 
$X\})$

Proposition 3.0.9. Given a metric space $(X, \rho)$ and $\alpha>0, \mu^{(\alpha)}: 2^{X} \rightarrow[0, \infty]$ is a metric exterior measure; therefore is a Borel measure.

Definition 3.0.10. For $A \subset X$, the Hausdorff dimension of $A$ is defined by

$$
\inf \left\{\alpha \geqslant 0: \mu^{(\alpha)}(A)=0\right\}=\sup \left\{\alpha \geqslant 0: \mu^{(\alpha)}(A)=\infty\right\}
$$

It is known (see [41]) that $\operatorname{dim}_{T}(K) \leqslant \operatorname{dim}_{H}(K)$.

Finally we introduce the notion of fractal dimension. If $K$ is a compact metric space let $N(r, K)$ be the least number of balls of radius $r$ necessary to cover $K$. The fractal dimension (or also known as capacity or box-counting dimension) $c(K)$ of $K$ is defined by:

$$
c(K)=\limsup _{r \rightarrow 0} \frac{\log N(r, K)}{\log (1 / r)}
$$

Alternatively, $c=c(K)$ is the least real number such that, for all $\epsilon>0$ there exists a $\delta>0$ with

$$
N(r, K) \leqslant\left(\frac{1}{r}\right)^{c+\epsilon}, 0<r<\delta
$$

From this, it is easy to see that

$$
\operatorname{dim}_{H}(K) \leqslant c(K)
$$

The fractal and Hausdorff dimension may differ significantly. One can easily see that the set $\left\{\frac{1}{n}: n \in \mathbb{N}^{*}\right\} \cup\{0\}$ is a compact subset of $\mathbb{R}$ with zero Hausdorff dimension and fractal dimension equal to $\frac{1}{2}$. It may even happen that the Hausdorff dimension is zero with the fractal dimension being infinite (see [31] for such an example).

\subsection{An estimate on the fractal dimension of attractors for gradient- like semigroups}

Recall that, from the definition, if $K \subset G$ are both compact subspaces of $X$, then $c(K) \leqslant$ $c(G)$.

Now assume that $X, Y$ are Banach spaces, $K \subset X, G \subset Y$ compact subsets and $f: K \rightarrow$ $G$ a Lipschitz function with Lipschitz constant $L_{f}>0$. Then $c(f(K)) \leqslant c(K)$. In fact, since 
$N(\epsilon, f(K)) \leqslant N\left(\epsilon / L_{f}, K\right)$ we have that

$$
\begin{aligned}
c(f(K)) & =\limsup _{\epsilon \rightarrow 0^{+}} \frac{\ln N(\epsilon, f(K))}{\ln (1 / \epsilon)} \leqslant \limsup _{\epsilon \rightarrow 0^{+}} \frac{\ln N\left(\epsilon / L_{f}, K\right)}{\ln (1 / \epsilon)} \\
& =\limsup _{\epsilon \rightarrow 0^{+}} \frac{\ln N\left(\epsilon / L_{f}, K\right)}{\ln \left(L_{f} / L_{f} \epsilon\right)}=\limsup _{\epsilon \rightarrow 0^{+}} \frac{\ln N\left(\epsilon / L_{f}, K\right)}{\ln \left(L_{f} / \epsilon\right)-\ln \left(L_{f}\right)} \\
& =\limsup _{\epsilon \rightarrow 0^{+}} \frac{1}{1-\frac{\ln \left(L_{f}\right)}{\ln \left(L_{f} / \epsilon\right)}} \frac{\ln N\left(\epsilon / L_{f}, K\right)}{\ln \left(L_{f} / \epsilon\right)} \leqslant c(K) .
\end{aligned}
$$

As a consequence of this result, if we assume the above hypotheses and in addition $X=Y$ and $K \subset f(K)$, then $c(K)=c(f(K))$.

Throughout this section we are interested in the calculation of the fractal dimension of the attractor, in terms of the fractal dimensions of the unstable manifolds associated to the isolated invariant sets. First we need to start with some results concerning the isolated invariant sets for a given gradient-like semigroup $\{T(t): t \geqslant 0\}$. The results of the following section are taken from [3], which is a product of this work.

Definition 3.1.1. Let $\{T(t): t \geqslant 0\}$ be a generalized gradient-like semigroup with global attractor $\mathscr{A}$, and $\Psi=\left\{\Xi_{1}, \cdots, \Xi_{n}\right\}$ a family of associated isolated invariant sets. We say that an isolated invariant set $\Xi_{i}$ is a source, if $W_{l o c}^{s}\left(\Xi_{i}\right) \cap \mathscr{A}=\Xi_{i}$; and a sink if $W^{u}\left(\Xi_{i}\right)=\Xi_{i}$. Otherwise, we say that $\Xi_{i}$ is a saddle.

Theorem 3.1.2. Let $\{T(t): t \geqslant 0\}$ be a generalized gradient-like semigroup with global attractor $\mathscr{A}$ and $\Psi=\left\{\Xi_{1}, \cdots, \Xi_{n}\right\}$ the associated isolated invariant sets. Then, there is at least one source and at least one sink.

Proof. Assume there are no sources. Then given $\Xi_{i}$, there exists a $\Xi_{j}(j \neq i)$ and a global solution $\xi$ such that

$$
\Xi_{i} \stackrel{t \rightarrow-\infty}{\longleftarrow} \xi(t) \stackrel{t \rightarrow \infty}{\longrightarrow} \Xi_{j}
$$

Inductively, we can construct a homoclinic structure since there is a finite number of isolated invariant sets, which leads us to a contradiction. A similar argument proves the existence of a sink.

Remark 3.1.3. Assume that $\{T(t): t \geqslant 0\}$ is a generalized gradient-like semigroup with global attractor $\mathscr{A}$ and $\Psi=\left\{\Xi_{1}, \cdots, \Xi_{n}\right\}$ the associated isolated invariant sets. We can easily show 
that the attractor $\mathscr{A}$ of $\{T(t): t \geqslant 0\}$ coincides with the attractor $\mathscr{A}^{\prime}$ of the discrete generalized gradient-like semigroup $\left\{S^{n}: n \in \mathbb{N}\right\}$, where $S=T(1)$. In fact, it is clear that $\mathscr{A} \subset \mathscr{A}^{\prime}$. Conversely, the attractor $\mathscr{A}^{\prime}$ is given as the union of unstable manifolds of the isolated invariant sets, and given a point $z \in \mathscr{A}^{\prime}$, there exists an isolated invariant set $\Xi_{i}$ and a global solution $\xi$ such that $\xi(0)=z$ and $\xi(-n) \stackrel{n \rightarrow \infty}{\rightarrow} \Xi_{i}$. Now, we can define $\phi(-t)$ for all $t \geqslant 0$ as follows: given $n \in \mathbb{N}$, define

$$
\phi(-t)=T(n-t) \xi(-n), \text { for all } 0 \leqslant t \leqslant n .
$$

This obviously gives us a global solution $\phi$ of $\{T(t): t \geqslant 0\}$ such that $\phi(0)=z$ and $\xi(-t) \stackrel{t \rightarrow \infty}{\rightarrow} \Xi_{i}$, which proves that $\mathscr{A}=\mathscr{A}^{\prime}$.

Due to this remark, we can now consider only the case of discrete generalized gradientlike semigroups and we begin stating our first result on fractal dimension.

Proposition 3.1.4. Let $\left\{T^{n}: n \in \mathbb{N}\right\}$ be a discrete semigroup with global attractor $\mathscr{A}$. Let $S=$ $T_{\left.\right|_{\mathscr{A}}}$ and assume that $S$ is Lipschitz continuous with Lipschitz constant $L>1$. Let $\left(A, A^{*}\right)$ be an attractor-repeller pair in $\mathscr{A}$, and assume that there exist constants $M \geqslant 1$ and $\omega>0$ such that, for all $K$ compact subset of $\mathscr{A}$ with $K \cap A^{*}=\varnothing$, we have $\operatorname{dist}_{\mathrm{H}}\left(S^{n} K, A\right) \leqslant M e^{-\omega n}$, for all $n \in \mathbb{N}$. Assume also that there is a neighborhood $B$ of $A^{*}$ in $\mathscr{A}$ such that $\bar{B} \cap A=\varnothing$.

Then

$$
c(B) \leqslant c(\mathscr{A}) \leqslant \max \left\{\frac{\omega+\ln (L)}{\omega} c(B), c(A)\right\} .
$$

Proof. Clearly, since $B \subset \mathscr{A}, c(B) \leqslant c(\mathscr{A})$. We only have to prove the right inequality. For this, we divide the proof into four steps:

Step 1: Define $\Omega_{n}=S^{n}(\mathscr{A} \backslash B) \backslash S^{n+1}(\mathscr{A} \backslash B)$, for all $n \in \mathbb{N}$. Note that $\Omega_{0}=(\mathscr{A} \backslash B) \backslash S(\mathscr{A} \backslash B) \subset$ $S(B) \backslash B \subset S(B)$ and therefore $c\left(\Omega_{0}\right) \leqslant c(S(B))=c(B)$, because $B \subset S(B)$ and $S$ is a Lipschitz continuous function.

Now we obtain an estimate on the minimum number of $r$-balls $N\left(r, \Omega_{k}\right)$ necessary to cover $\Omega_{k}$ in terms of the numbers of balls necessary to cover $\Omega_{0}$. Let $n_{0}^{r, k}=N\left(r / L^{k}, \Omega_{0}\right)$ and $\left\{x_{1}, \ldots, x_{n_{0}^{r, k}}\right\}$ a finite sequence of points in $\Omega_{0}$ such that

$$
\Omega_{0} \subset \bigcup_{i=1}^{n_{0}^{r, k}} B\left(x_{i}, r / L^{k}\right) .
$$


Set, for each $i=1, \ldots, n_{0}^{r, k}, \xi_{i}=S^{k}\left(x_{i}\right) \in \Omega_{k}$. Then, for each $y \in \Omega_{k}$ there exists $z \in \Omega_{0}$ such that $y=S^{k}(z), z \in B\left(x_{i}, r / L^{k}\right)$ for some $i=1, \ldots, n_{0}^{r, k}$ and we have

$$
\left\|y-\xi_{i}\right\|=\left\|S^{k}(z)-S^{k}\left(x_{i}\right)\right\| \leqslant L^{k}\left\|z-x_{i}\right\|<r \text {, for all } y \in \Omega_{k} .
$$

So, we just proved $\Omega_{k} \subset \cup_{i=1}^{n_{0}^{r, k}} B\left(\xi_{i}, r\right)$, which gives $N\left(r, \Omega_{k}\right) \leqslant n_{0}^{r, k}$.

Step 2: Given $r>0$, since $\operatorname{dist}_{\mathrm{H}}\left(S^{n}(\mathscr{A} \backslash B), A\right) \leqslant M e^{-\omega n}$ for all $n \geqslant 0$, there exists a $n_{0}(r)=$ $\left\lceil\frac{1}{\omega} \ln \left(\frac{M}{r}\right)\right\rceil$ such that

$$
G(r):=\left(\bigcup_{j \geqslant n_{0}(r)} \Omega_{j}\right) \cup A \subset \mathscr{O}_{r}(A),
$$

where $\mathscr{O}_{r}(A)$ denotes the $r$-neighborhood of $A$. So, if $A \subset \cup_{i=1}^{N(r, A)} B\left(x_{i}, r\right)$ with $x_{i} \in A$ for all $i=1, \ldots, N(r, A)$, then $\mathscr{O}_{r}(A) \subset \cup_{i=1}^{N(r, A)} B\left(x_{i}, 2 r\right)$ therefore $N\left(2 r, \mathscr{O}_{r}(A)\right) \leqslant N(r, A)$. We conclude that $N\left(r, G\left(\frac{r}{2}\right)\right) \leqslant N\left(\frac{r}{2}, A\right)$.

Step 3: From Step 1, if $H(r):=\bigcup_{j=0}^{n_{0}(r)} \Omega_{j}$ we have

$$
N(r, H(r)) \leqslant n_{0}(r) \max _{k=0, \ldots, n_{0}(r)} N\left(r / L^{k}, \Omega_{0}\right)=n_{0} N\left(r / L^{n_{0}(r)}, \Omega_{0}\right)
$$

since $L>1$.

Step 4: First, note that for each $r>0$, we have that $\mathscr{A}=B \cup G\left(\frac{r}{2}\right) \cup H\left(\frac{r}{2}\right)$ and therefore

$$
\begin{aligned}
N(r, \mathscr{A}) & \leqslant 3 \max \{N(r, B) ; N(r, H(r / 2)) ; N(r, G(r / 2))\} \\
& \leqslant 3 \max \{N(r, B) ; N(r / 2, H(r / 2)) ; N(r / 2, A)\} \\
& \leqslant 3 \max \left\{N(r, B) ; n_{0}(r / 2) N\left(r / L^{n_{0}(r / 2)}, \Omega_{0}\right) ; N(r / 2, A)\right\} .
\end{aligned}
$$

Since the logarithm function is increasing, we obtain

$$
\ln N(r, \mathscr{A}) \leqslant \ln 3+\max \left\{\ln N(r, B) ; \ln n_{0}(r / 2)+\ln N\left(r / L^{n_{0}(r / 2)}, \Omega_{0}\right) ; \ln N(r / 2, A)\right\} .
$$


Hence

$\frac{\ln N(r, \mathscr{A})}{\ln (1 / r)} \leqslant \frac{\ln 3}{\ln (1 / r)}+\max \left\{\frac{\ln N(r, B)}{\ln (1 / r)} ; \frac{\ln n_{0}(r / 2)}{\ln (1 / r)}+\frac{\ln N\left(r / L^{n_{0}(r / 2)}, \Omega_{0}\right)}{\ln (1 / r)} ; \frac{\ln N(r / 2, A)}{\ln (1 / r)}\right\}$

Obviously, $\limsup _{r \rightarrow 0^{+}} \frac{\ln 3}{\ln (1 / r)}=0$. Now, we compute the other terms:

(a)

$$
\limsup _{r \rightarrow 0^{+}} \frac{\ln n_{0}(r / 2)}{\ln (1 / r)}=\limsup _{r \rightarrow 0^{+}} \frac{\ln 1 / \omega}{\ln (1 / r)}+\limsup _{r \rightarrow 0^{+}} \frac{\ln (\ln (2 M / r))}{\ln (1 / r)}=0
$$

(b)

$$
\begin{aligned}
\limsup _{r \rightarrow 0^{+}} \frac{\ln N\left(r / L^{n_{0}(r / 2)}, \Omega_{0}\right)}{\ln (1 / r)} & =\limsup _{r \rightarrow 0^{+}} \frac{\ln N\left(r / L^{n_{0}(r / 2)}, \Omega_{0}\right)}{\ln \left(L^{n_{0}(r / 2)} / r L^{n_{0}}\right)} \\
& =\limsup _{r \rightarrow 0^{+}} \frac{1}{1-\frac{n_{0}(r / 2) \ln L}{\ln \left(L^{n_{0}(r / 2)} / r\right)}} \frac{\ln N\left(r / L^{n_{0}(r / 2)}, \Omega_{0}\right)}{\ln \left(L^{n_{0}(r / 2)} / r\right)}
\end{aligned}
$$

but

$$
\limsup _{r \rightarrow 0^{+}} \frac{1}{1-\frac{n_{0}(r / 2) \ln L}{\ln \left(L^{\left.n_{0}(r / 2) / r\right)}\right.}}=\limsup _{r \rightarrow 0^{+}}\left(\frac{n_{0}(r / 2) \ln (L)}{\ln (1 / r)}+1\right),
$$

and since $\frac{1}{\omega} \ln \left(\frac{2 M}{r}\right) \leqslant n_{0} \leqslant \frac{1}{\omega} \ln \left(\frac{2 M}{r}\right)+1$,

$$
\underset{r \rightarrow 0^{+}}{\limsup }\left(\frac{n_{0}(r / 2) \ln (L)}{\ln (1 / r)}+1\right)=\frac{\omega+\ln (L)}{\omega},
$$

which shows that

$$
\limsup _{r \rightarrow 0^{+}} \frac{\ln N\left(r / L^{n_{0}(r / 2)}, \Omega_{0}\right)}{\ln (1 / r)} \leqslant \frac{\omega+\ln (L)}{\omega} c\left(\Omega_{0}\right)
$$

(c)

$$
\begin{aligned}
\underset{r \rightarrow 0^{+}}{\limsup } \frac{\ln N(r / 2, A)}{\ln (1 / r)} & =\underset{r \rightarrow 0^{+}}{\limsup \frac{\ln N(r / 2, A)}{\ln (2 / 2 r)}} \\
& \limsup _{r \rightarrow 0^{+}} \frac{1}{1+\frac{\ln (1 / 2)}{\ln (1 / r)}} \frac{\ln N(r / 2, A)}{\ln (2 / r)} \leqslant c(A) .
\end{aligned}
$$


Putting together (a), (b) and (c), we obtain

$$
c(\mathscr{A}) \leqslant \max \left\{c(B), \frac{\omega+\ln (L)}{\omega} c\left(\Omega_{0}\right), c(A)\right\} \leqslant \max \left\{\frac{\omega+\ln (L)}{\omega} c(B), c(A)\right\} .
$$

Here we use the fact that $c\left(\Omega_{0}\right) \leqslant c(B)$. The proof is now complete.

Now, using this proposition we can estimate the fractal dimension of a global attractor of a discrete generalized gradient-like semigroup $\left\{T^{n}: n \in \mathbb{N}\right\}$ in terms of the fractal dimensions of the local unstable manifolds of the isolated invariant sets.

Theorem 3.1.5. Let $\left\{T^{n}: n \in \mathbb{N}\right\}$ be a discrete generalized gradient-like semigroup with global attractor $\mathscr{A}$ and $\Psi=\left\{\Xi_{1}, \ldots, \Xi_{p}\right\}$ the associated isolated invariant sets. Assume that the restriction $T_{\left.\right|_{\mathscr{A}}}$ to $\mathscr{A}$ of the operator $T$ is a Lipschitz continuous function with Lipschitz constant $L>1$ and assume also that there exist constants $M>1$ and $\omega>0$ such that for every attractorrepeller pair $\left(A, A^{*}\right)$ in $\mathscr{A}$ and every compact subset $K \subset \mathscr{A}$ with $K \cap A^{*}=\varnothing$ we have

$$
\operatorname{dist}_{\mathrm{H}}\left(T^{n}(K), A\right) \leqslant M e^{-\omega n}, \text { for all } n \geqslant 0 \text {. }
$$

Finally, assume that the local unstable manifolds $\left\{W_{l o c}^{u}\left(\Xi_{i}\right), i, \ldots, p\right\}$ are given as graphs of Lipschitz functions. Under these conditions

$$
\max _{i=1, \ldots, p} c\left(W_{l o c}^{u}\left(\Xi_{i}\right)\right) \leqslant c(\mathscr{A}) \leqslant \frac{\omega+\ln (L)}{\omega} \max _{i=1, \ldots, p} c\left(W_{l o c}^{u}\left(\Xi_{i}\right)\right)
$$

Proof. Since $\left\{T^{n}: \quad n \in \mathbb{N}\right\}$ is a discrete gradient-like semigroup, there exists at least one source. Let $\Xi_{i}$ be one of these sources and $B_{i}$ a neighborhood of $\Xi_{i}$ in $\mathscr{A}$ such that $B_{i} \subset$ $W_{l o c}^{u}\left(\Xi_{i}\right)$ and $T\left(B_{i}\right) \subset W_{l o c}^{u}\left(\Xi_{i}\right)$, so that $c\left(B_{i}\right)=c\left(T\left(B_{i}\right)\right)=c\left(W_{l o c}^{u}\left(\Xi_{i}\right)\right)$. Now, it is easy to see that $\Xi_{i}=A_{i}^{*}$, where $A_{i}=\cup_{j \neq i} W_{l o c}^{u}\left(\Xi_{j}\right)$. By Proposition 3.1.4,

$$
c\left(B_{i}\right) \leqslant c(\mathscr{A}) \leqslant \max \left\{\frac{\omega+\ln (L)}{\omega} c\left(B_{i}\right), c\left(A_{i}\right)\right\}
$$

that is

$$
c\left(W_{l o c}^{u}\left(\Xi_{i}\right)\right) \leqslant c(\mathscr{A}) \leqslant \max \left\{\frac{\omega+\ln (L)}{\omega} c\left(W_{l o c}^{u}\left(\Xi_{i}\right)\right), c\left(A_{i}\right)\right\} .
$$

Now, restrict the operator $T$ to the attractor $A_{i}$. Thus, we have a discrete generalized gradient- 
like semigroup with attractor $A$ and $\Xi^{\mathbf{1}}=\Psi \backslash\left\{\Xi_{i}\right\}$, which has at least one source $\Xi_{k}$, with $k \neq i$. We can use the same argument above to prove that

$$
c\left(W_{l o c}^{u}\left(\Xi_{k}\right)\right) \leqslant c\left(A_{i}\right) \leqslant \max \left\{\frac{\omega+\ln (L)}{\omega} c\left(W_{l o c}^{u}\left(\Xi_{k}\right)\right), c\left(A_{k}\right)\right\} .
$$

And joining these two results, we obtain

$$
\max _{j=i, k} c\left(W_{l o c}^{u}\left(\Xi_{j}\right)\right) \leqslant c(\mathscr{A}) \leqslant \max \left\{\frac{\omega+\ln (L)}{\omega} c\left(W_{l o c}^{u}\left(\Xi_{i}\right)\right), \frac{\omega+\ln (L)}{\omega} c\left(W_{l o c}^{u}\left(\Xi_{k}\right)\right), c\left(A_{k}\right)\right\} .
$$

This process must stop, since there are just a finite number of isolated invariant sets, and proceeding inductively we obtain the desired result.

\section{Remark 3.1.6.}

(a) The proof of this theorem suggests a certain order in the family of isolated invariant sets and, after a possible index rearrangement, we can assume that $\Psi=\left\{\Xi_{1}, \ldots, \Xi_{p}\right\}$ and in the proof, the first source in $\mathscr{A}$ to be chosen is $\Xi_{p}$, the second is $\Xi_{p-1}$ and so on. Such an ordering can be used to form a new family $\mathscr{N}=\left\{\mathscr{N}_{1}, \ldots, \mathscr{N}_{m}\right\}$ with $m \leqslant p$ called energy level decomposition for the attractor $\mathscr{A}$, which is a Morse decomposition for $\mathscr{A}$. For more details see Section 5 of [1]. Using this decomposition we can see that the fractal dimension of the sets $W_{\text {loc }}^{u}\left(\Xi_{i}\right)$ is a non-increasing function of the index $i$, and we have that

$$
c\left(W_{l o c}^{u}\left(\Xi_{1}\right)\right) \leqslant c(\mathscr{A}) \leqslant \frac{\omega+\ln (L)}{\omega} c\left(W_{l o c}^{u}\left(\Xi_{1}\right)\right) .
$$

(b) We already knew that in this case the Hausdorff dimension of $\mathscr{A}$ is finite and thus the topological dimension of $\mathscr{A}$ is also finite, which allows us to embed $\mathscr{A}$ in $\mathbb{R}^{2 \operatorname{dim}_{T}(\mathscr{A})+1}$. Now we can embed $\mathscr{A}$ in an Euclidian space of higher dimension, but with a projection with Hölder continuous inverse.

Our next result is an immediate corollary of the preceding theorem, once we recall some basic facts concerning discrete gradient-like semigroups $\left\{T^{n}: n \in \mathbb{N}\right\}$ with an attractor $\mathscr{A}$ and a finite set $\mathscr{E}=\left\{e_{1}, \ldots, e_{p}\right\}$ of fixed hyperbolic points. First, the reader can check (see [19] for a proof) that the local unstable (stable) manifold $W_{l o c}^{u}\left(e_{i}\right)\left(W_{l o c}^{s}\right)$ is given by a graph of a Lipschitz function. Now, in these conditions it is easy to see that there are only a finite 
number of attractor-repeller pairs $\left(A, A^{*}\right)$, namely, the pairs $\left(A, A^{*}\right)$, with

$$
A=\bigcup_{\substack{i \in I \\ I \subset\{1, \ldots, p\}}} W^{u}\left(e_{i}\right)
$$

Using this fact and the exponential attraction of each fixed point, we can prove that there exist constants $M \geqslant 1$ and $\omega>0$ such that for every attractor-repeller pair $\left(A, A^{*}\right)$ and every compact subset $K$ of $\mathscr{A}$ with $K \cap A^{*}=\varnothing$, we have

$$
\operatorname{dist}_{H}\left(T^{n}(K), A\right) \leqslant M e^{-\omega n} \text {, for all } n \geqslant 0 \text {. }
$$

From these two facts it follows the next result:

Corollary 3.1.7. Let $\left\{T^{n}: n \in \mathbb{N}\right\}$ be a discrete gradient-like semigroup with an attractor $\mathscr{A}$ and a finite set $\mathscr{E}=\left\{e_{1}, \ldots, e_{p}\right\}$ of fixed hyperbolic points. Assume that the restriction of $T$ to $\mathscr{A}$ is a Lipschitz function with Lipschitz constant $L>1$. Let $M \geqslant 1$ and $\omega>0$ be two constants such that for every attractor-repeller pair $\left(A_{i}, A_{i}^{*}\right)$, with $A_{i}=\cup_{j \neq i} W_{l o c}^{u}\left(e_{j}\right)$ and every compact subset $K$ of $\mathscr{A}$ with $K \cap A^{*}=\varnothing$ we have

$$
\operatorname{dist}_{H}\left(T^{n}(K), A\right) \leqslant M e^{-\omega n}, \text { for all } n \geqslant 0 \text {. }
$$

Then

$$
\max _{i=1, \ldots, p} c\left(W_{l o c}^{u}\left(e_{i}\right)\right) \leqslant c(\mathscr{A}) \leqslant \frac{\omega+\ln (L)}{\omega} \max _{i=1, \ldots, p} c\left(W_{l o c}^{u}\left(e_{i}\right)\right) .
$$

Under similar, although appropriately modified, hypotheses it is possible to show an analogous result to Proposition 3.1.4, but using now local stable manifolds.

Proposition 3.1.8. Let $\left\{T^{n}: n \in \mathbb{N}\right\}$ be a discrete semigroup with global attractor $\mathscr{A}$. Let $S=T_{\left.\right|_{A}}$ and assume that $S$ is invertible with inverse $S^{-1}$ a Lipschitz continuous map, with Lipschitz constant $L>1$. Let $\left(A, A^{*}\right)$ be an attractor-repeller pair in $\mathscr{A}$, and assume that there exist constants $M \geqslant 1$ and $\omega>0$ such that, for all $K$ compact subset of $\mathscr{A}$ with $K \cap A=\varnothing$, we have $\operatorname{dist}_{\mathrm{H}}\left(S^{-n} K, A^{*}\right) \leqslant M e^{-\omega n}$, for all $n \in \mathbb{N}$. Assume also that there is a neighborhood $B$ of $A$ in $\mathscr{A}$ such that $\bar{B} \cap A^{*}=\varnothing$.

Then

$$
c(B) \leqslant c(\mathscr{A}) \leqslant \max \left\{\frac{\omega+\ln (L)}{\omega} c(B), c\left(A^{*}\right)\right\} .
$$


Additionally we can also establish the next result.

Theorem 3.1.9. Let $\left\{T^{n}: n \in \mathbb{N}\right\}$ be a discrete generalized gradient-like semigroup with global attractor $\mathscr{A}$ and $\Psi=\left\{\Xi_{1}, \ldots, \Xi_{p}\right\}$ the associated isolated invariant sets. Assume that the restriction $T_{\left.\right|_{A}}$ to $\mathscr{A}$ of the operator $T$ is invertible with its inverse $T^{-1}$ a Lipschitz continuous function with Lipschitz constant $L>1$ and assume also that there exist constants $M>1$ and $\omega>0$ such that for every attractor-repeller pair $\left(A, A^{*}\right)$ in $\mathscr{A}$ and every compact subset $K \subset \mathscr{A}$ with $K \cap A=\varnothing$ we have

$$
\operatorname{dist}_{\mathrm{H}}\left(T^{-n}(K), A^{*}\right) \leqslant M e^{-\omega n}, \text { for all } n \geqslant 0 \text {. }
$$

Finally, assume that the intersection of the local stable manifolds $\left\{W_{l o c}^{s}\left(\Xi_{i}\right), i, \ldots, p\right\}$ with the global attractor $\mathscr{A}$ are given as graphs of Lipschitz functions. Under these conditions

$$
\max _{i=1, \ldots, p} c\left(W_{l o c}^{s}\left(\Xi_{i}\right) \cap \mathscr{A}\right) \leqslant c(\mathscr{A}) \leqslant \frac{\omega+\ln (L)}{\omega} \max _{i=1, \ldots, p} c\left(W_{l o c}^{s}\left(\Xi_{i}\right) \cap \mathscr{A}\right) .
$$

Remark 3.1.10. If the hypotheses of Corollary 3.1.7 are satisfied, $S$ is invertible and the hypotheses of exponential attraction for the inverse to the local repellers are also satisfied then

$$
c(\mathscr{A}) \leqslant \min \left\{\frac{\omega+\ln L}{\omega} \max _{i=1, \cdots, p} c\left(W_{l o c}^{u}\left(e_{i}\right)\right), \max _{i=1, \cdots, p} c\left(W_{l o c}^{s}\left(e_{i}\right) \cap \mathscr{A}\right)\right\} .
$$

This can be easily seen if we return to the proof of Proposition 3.1.4. If S is Lipschitz continuous with Lipschitz constant $L>1$, then $S^{-1}$ is also Lipschitz with Lipschitz constant $1 / L<1$, and the proof in this case is modified. More precisely, in Step 3,

$$
N(r, H(r)) \leqslant n_{0} N\left(r, \Omega_{0}\right)
$$

Also, with the reversed hypotheses

$$
c(\mathscr{A}) \leqslant \min \left\{\max _{i=1, \cdots, p} c\left(W_{l o c}^{u}\left(e_{i}\right)\right), \frac{\omega+\ln L}{\omega} \max _{i=1, \cdots, p} c\left(W_{l o c}^{s}\left(e_{i}\right) \cap \mathscr{A}\right)\right\} .
$$


Remark 3.1.11. Consider the autonomous equation

$$
u_{t}=u_{x x}+\lambda\left(u-\beta u^{3}\right)
$$

for $x \in[0, \pi]$ with Dirichlet boundary conditions. We consider the family of attractors $\left\{\mathscr{A}_{\lambda}\right.$ : $\lambda>0\}$, varying with the parameter $\lambda$. Note that our argument implies that if we approach a bifurcation point $\lambda=n^{2}, n \in \mathbb{N}$, our estimate on the fractal dimension of the attractor $\mathscr{A}_{\lambda}$ explodes, since the rate of exponential attraction $\omega$ approaches to zero (see [22] where it is proved that this attraction is in fact polynomial). However, we know that the fractal dimension of the above Chafee-Infante equation is finite and of order $\sqrt{\lambda}$ for all values of $\lambda \geq \lambda_{1}$ (the first eigenvalue of the Laplacian operator) (see, for instance, [46, 41]). Despite of this fact, if we choose $\lambda^{\prime}$ near $\lambda\left(\lambda^{\prime}>\lambda\right)$, then the estimate is finite, as we have the exponential attraction to hyperbolic equilibria. Moreover, for any sequence $\left\{\lambda_{n}: n \in \mathbb{N}\right\}$ such that $\lambda_{n}$ is away from the endpoints of the interval $\left(n^{2},(n+1)^{2}\right)$, uniformly for $n \in \mathbb{N}$, our estimate is of order $\sqrt{\lambda_{n}}$.

\subsection{Non-autonomous dynamical systems and attractor-repeller pairs}

In this section we are interested in obtaining an estimate for the fractal dimension of a pullback attractor for a gradient-like evolution process. We now will define attractor-repeller pairs for evolution processes and extract some of their properties. For this purpose we follow the ideas in [24], and some demonstrations are omitted since they can be found in this reference.

To make the theory a little bit more general, we will consider non-autonomous dynamical systems (which we define below).

Definition 3.2.1. Let $\left(X, d_{X}\right)$ and $\left(P, d_{P}\right)$ be metric spaces. A non-autonomous dynamical system (NDS), denoted by $(\theta, \varphi)$ or shortly $\varphi$ when there is no confusion, consists of two ingredients:

(i) A model of the non-autonomous driving system, namely a dynamical system $\theta$ on $P$ 
with time set $\mathbb{T}=\mathbb{Z}$ or $\mathbb{R} ;$ i.e.

$$
\begin{gathered}
\theta_{0} p=p, \text { for all } p \in P, \\
\theta_{t+s} p=\theta_{t}\left(\theta_{s} p\right), \text { for all } p \in P, t, s \in \mathbb{T},
\end{gathered}
$$

and the map $(t, p) \mapsto \theta_{t} p$ is continuous.

(ii) A model of the non-autonomously perturbed system, namely a cocycle $\varphi$ over $\theta$; i.e. a continuous mapping

$$
\varphi: \mathbb{T}^{+} \times P \times X \rightarrow X,(t, p, x) \mapsto \varphi(t, p, x)
$$

such that the family $\varphi(t, p, \cdot)=\varphi(t, p): X \rightarrow X$ of self-mappings of $X$ satisfies the cocycle property:

$$
\begin{gathered}
\varphi(0, p)=\mathrm{id}_{X}, \text { for all } p \in P, \\
\varphi(t+s, p)=\varphi\left(t, \theta_{s} p\right) \circ \varphi(s, p), \text { for all } t, s, \in \mathbb{T}^{+}, p \in P .
\end{gathered}
$$

$P$ is called the base space and $X$ is the state space.

Remark 3.2.2. Given an evolution process $\{S(t, s):(t, s) \in \mathscr{P}\}$, then we can construct a nonautonomous dynamical system with base space $P=\mathbb{R}$ by setting

$$
\varphi(t, s) \doteq S(t+s, s), \text { for all } t \geqslant 0 \text { and } s \in \mathbb{R}
$$

thus the non-autonomous dynamical systems are a generalization of evolution processes, and every result done from now on can be applied for evolution processes.

Definition 3.2.3. A family of subsets $\hat{D}=\{D(p)\}_{p \in P}$ of $X$ is called a non-autonomous set. If each fiber $D(p)$ is closed/compact/open, then $\hat{D}$ is called a non-autonomous closed/compact/open set.

Definition 3.2.4. A non-autonomous set $\hat{D}$ is said to be forward invariant under the NDS $\varphi$ if $\varphi(t, p) D(p) \subset D\left(\theta_{t} p\right)$ for all $p \in P$ and $t \geqslant 0$. It is said to be invariant if $\varphi(t, p) D(p)=D\left(\theta_{t} p\right)$ for all $p \in P$ and $t \geqslant 0$. 
Definition 3.2.5. Assume that $\hat{D}$ is a non-autonomous set, then the (pullback) omega-limit set of $\hat{D}, \omega_{\hat{D}}$, is defined by

$$
\omega_{\hat{D}}(p):=\bigcap_{t \geqslant 0} \overline{\bigcup_{s \geqslant t} \varphi\left(s, \theta_{-s}\right) D\left(\theta_{-s} p\right)}, \text { for each } p \in P
$$

To simplify the notation, we will write $\omega_{D}$ instead of $\omega_{\hat{D}}$.

Definition 3.2.6. Given two non-autonomous sets $\hat{D}$ and $\hat{A}$, we say that $\hat{A}$ pullback attracts $\hat{D}$ if

$$
\lim _{t \rightarrow \infty} \operatorname{dist}_{H}\left(\varphi\left(t, \theta_{-t} p\right) D\left(\theta_{-t} p\right), A(p)\right)=0
$$

holds for all $p \in P$, where $\mathrm{d}(A, B)$ stands for the Hausdorff semi-distance between two sets $A$ and $B$, i.e. $\mathrm{d}(A, B):=\sup _{x \in A} \inf _{y \in B} d_{x}(x, y)$. As usual, we set $\mathrm{d}(\varnothing, A)=0$ and $\mathrm{d}(A, \varnothing)=\infty$, for a nonempty set $A$.

Remark 3.2.7. 1. Clearly $x \in \omega_{D}(p)$ if and only if there exist sequences $t_{n} \rightarrow \infty$ and $x_{n} \in$ $D\left(\theta_{-t_{n}} p\right)$ such that $\varphi\left(t_{n}, \theta_{-t_{n}} p\right) x_{n} \rightarrow x$ as $n \rightarrow \infty$.

2. For any two non-autonomous sets $\hat{D}_{1}$ and $\hat{D}_{2}$, we have

$$
\omega_{D_{1} \cup D_{2}}(p)=\omega_{D_{1}}(p) \cup \omega_{D_{2}}(p)
$$

for each $p \in P$.

3. If a non-autonomous closed set $\hat{E}$ pullback attracts another $\hat{D}$, then $\omega_{D}(p) \subset E(p)$ for all $p \in P$.

Definition 3.2.8. Let $\varphi$ be a NDS. For a fixed $p \in P$ and $x \in X$, a mapping $\sigma .(p): \mathbb{R} \rightarrow X$ is called an entire $p$-orbit through $x$ of $\varphi$ if it satisfies the cocycle property

$$
\left\{\begin{array}{l}
\sigma_{0}(p)=x \\
\sigma_{t+s}(p)=\varphi\left(t, \theta_{s} p\right) \sigma_{s}(p), \quad \text { for all } t \geqslant 0, s \in \mathbb{R} .
\end{array}\right.
$$

By the cocycle property of $\sigma$, it is clear that for arbitrary $t \geqslant 0$ and $p \in P$ we have

$$
\sigma_{t}(p)=\varphi(t, p) \sigma_{0}(p)
$$


Lemma 3.2.9. Assume that $\hat{D}$ is a forward invariant non-autonomous compact set, then for any $p \in P$ and any point $x \in \omega_{D}(p)$ there exists an entire $p$-orbit $\sigma .(p)$ through $x$ such that $\sigma_{t}(p) \in \omega_{D}\left(\theta_{t} p\right)$ for all $t \in \mathbb{R}$.

Proof: Let $p \in P$ fixed and $x \in \omega_{D}(p)$. Define $\sigma_{0}(p)=x$ and for $t \geqslant 0, \sigma_{t}(p):=\varphi(t, p) \sigma_{0}(p)$. It is clear that $\sigma_{t}(p) \in \omega_{D}\left(\theta_{t} p\right)$ for all $t \geqslant 0$, by the invariance of $\omega_{D}$. Now, by definition of the omega-limit set, there exist sequences $t_{n} \rightarrow \infty$ and $x_{n} \in D\left(\theta_{-t_{n}} p\right)$ such that $\varphi\left(t_{n}, \theta_{-t_{n}} p\right) x_{n} \rightarrow$ $x, n \rightarrow \infty$. By the forward invariance of $\hat{D}$, for each $k \in \mathbb{Z}^{-}$, there exists $N_{0} \in \mathbb{N}$ such that

$$
\varphi\left(t_{n}+k, \theta_{-t_{n}} p\right) x_{n} \in D\left(\theta_{k} p\right), \text { for all } n \geqslant N_{0}
$$

Using the compactness of $\hat{D}$ and a diagonalization process, we can find a subsequence $n_{m} \rightarrow \infty$ and points $\tilde{x}_{k} \in D\left(\theta_{k} p\right)$ such that

$$
\varphi\left(t_{n_{m}}+k, \theta_{-t_{n_{m}}} p\right) x_{n_{m}} \rightarrow \tilde{x}_{k}
$$

for each $k \in \mathbb{Z}^{-}$. Define, for $k \leqslant t \leqslant k+1, \sigma_{t}(p)=\varphi\left(t-k, \theta_{k} p\right) \tilde{x}_{k}$. In this way it is easy to see that $\sigma .(p): \mathbb{R} \rightarrow X$ is an entire $p$-orbit through $x$ such that $\sigma_{t}(p) \in \omega_{D}\left(\theta_{t} p\right)$ for all $t \in \mathbb{R}$.

Corollary 3.2.10. Assume that $\hat{D}$ is an invariant non-autonomous compact set. Then for any $p \in P$ and any point $x \in D(p)$, there exists an entire $p$-orbit $\sigma .(p)$ through $x$ that lies in $\hat{D}$, i.e. $\sigma_{t}(p) \in D\left(\theta_{t} p\right)$ for all $t \in \mathbb{R}$.

Definition 3.2.11. Assume that $\varphi$ is an NDS with base space $P$ and state space $X$. An universe $\hat{\mathscr{D}}$ is a collection of nonempty non-autonomous sets which is closed with respect to set inclusion, i.e. if $\hat{D}_{1} \in \hat{\mathscr{D}}$ and $\hat{D}_{2} \subset \hat{D}_{1}$ then $\hat{D}_{2} \in \hat{\mathscr{D}}$. A non-autonomous compact set $\hat{S} \in \hat{\mathscr{D}}$ is called a $\hat{\mathscr{D}}$-pullback attractor of $\varphi$ if

(i) $\hat{S}$ is invariant;

(ii) $\hat{S}$ is $\hat{\mathscr{D}}$-pullback attracting, i.e. $\hat{S}$ pullback attracts every $\hat{D} \in \hat{\mathscr{D}}$.

A continuous function $\xi: P \rightarrow X$ is called a global solution for the non-autonomous dynamical system $\varphi$ if it satisfies

$$
\varphi\left(t, \theta_{-t} p\right) \xi\left(\theta_{-t} p\right)=\xi(p) \text {, for all } t \in \mathbb{T}^{+} \text {and } p \in P \text {. }
$$


Proposition 3.2.12. Given an attraction universe $\hat{\mathscr{D}}$, the pullback attractor with respect to $\hat{\mathscr{D}}$ is unique.

Proof. Let $\hat{A}$ and $\hat{A}^{\prime}$ be two pullback attractors with respect to the attraction universe $\hat{\mathscr{D}}$. Since $\hat{A}^{\prime} \in \mathscr{D}$, we have for every $p \in P$ that

$$
\operatorname{dist}_{H}\left(A^{\prime}(p), A(p)\right)=\lim _{t \rightarrow \infty} \operatorname{dist}_{H}\left(\varphi\left(t, \theta_{-t} p\right) A^{\prime}\left(\theta_{-t} p\right), A(p)\right)=0
$$

and since $A^{\prime}(p)$ and $A(p)$ are both compact, it follows that $A^{\prime}(p) \subseteq A(p)$. Analogously we show that $A(p) \subseteq A^{\prime}(p)$ which concludes the result.

Definition 3.2.13 (Pullback absorbing set with respect to an attraction universe). Let $\hat{\mathscr{D}}$ be an attraction universe of a NDS $\varphi$. A non-autonomous set $\hat{B} \in \hat{\mathscr{D}}$ is called pullback absorbing with respect to $\hat{\mathscr{D}}$ iffor each $\hat{D} \in \hat{\mathscr{D}}$ and $p \in P$ there exists $a t_{0} \geqslant 0$ such that

$$
\varphi\left(t, \theta_{-t} p\right) D\left(\theta_{-t} p\right) \subset B(p), \text { for all } t \geqslant t_{0} .
$$

Theorem 3.2.14 (Existence of a pullback attractor with respect to an attraction universe). Let $\varphi$ be a NDS and assume that the non-autonomous compact set $\hat{B}$ is pullback absorbing with respect to an attraction universe $\hat{\mathscr{D}}$. Then there exists a pullback attractor $\hat{A}$ with respect to $\hat{\mathscr{D}}$, where for each $p \in P$, the fibers $A(p)$ are defined by

$$
A(p)=\omega_{B}(p)
$$

Proof. Let $\hat{A}$ be defined by (3.2.3). Firstly, we will show that for every $p \in P$

$$
\lim _{t \rightarrow \infty} \operatorname{dist}_{H}\left(\varphi\left(t, \theta_{-t} p\right) B\left(\theta_{-t} p\right), A(p)\right)=0
$$

Assume to contrary that there exist a $p \in P$, a sequence $\left\{t_{n}\right\}_{n \geqslant 0} \subseteq[0, \infty)$ with $t_{n} \rightarrow \infty$ as $n \rightarrow$ $\infty$, a sequence $\left\{x_{n}\right\}_{n \geqslant 0}$ with $x_{n} \in B\left(\theta_{-t_{n}} p\right)$ and an $\epsilon>0$ such that

$$
\operatorname{dist}_{H}\left(\varphi\left(t_{n}, \theta_{-t_{n}} p\right) x_{n}, A(p)\right) \geqslant \epsilon \text {, for every } n \geqslant 0 \text {. }
$$


Since $\hat{B}$ is an absorbing family with respect to $\hat{\mathscr{D}}, \hat{B} \in \hat{\mathscr{D}}$ and $t_{n} \rightarrow \infty$ as $n \rightarrow \infty$, we can assume that $y_{n} \doteq \varphi\left(t_{n}, \theta_{-t_{n}} p\right) x_{n} \in B(p)$ for every $n \geqslant 0$. By the compactness of $B(p)$, we can also assume that there exists $y \in B(p)$ such that the sequence $\left\{y_{n}\right\}_{n \geqslant 0}$ satisfies $y_{n} \rightarrow y$ as $n \rightarrow \infty$.

But $\operatorname{dist}_{H}\left(y_{n}, A(p)\right) \geqslant \epsilon$ for every $n \geqslant 0$ and so $\operatorname{dist}_{H}(y, A(p)) \geqslant \epsilon$, which is a contradiction since $y \in \omega_{B}(p)=A(p)$.

Now let $\hat{D} \in \hat{\mathscr{D}}$. The above calculation gives us that, given $p \in P$ and $\epsilon>0$, there exists $t_{0} \geqslant 0$ such that

$$
\operatorname{dist}_{H}\left(\varphi\left(t, \theta_{-t} p\right) B\left(\theta_{-t} p\right), A(p)\right)<\epsilon, \text { for all } t \geqslant t_{0}
$$

Now, the family $\hat{B}$ is pullback absorbing with respect to $\hat{\mathscr{D}}$ and so, for the $t_{0}$ given above, there exists $t_{1} \geqslant 0$ such that $\varphi\left(t, \theta_{-t+t_{0}} p\right) D\left(\theta_{-t+t_{0}} p\right) \subset B\left(\theta_{t_{0}} p\right)$ for every $t \geqslant t_{1}$.

Thus, for $t \geqslant t_{1}$ we have

$$
\begin{aligned}
\operatorname{dist}_{H}\left(\varphi\left(t, \theta_{-t} p\right) D\left(\theta_{-t} p\right), A(p)\right) & =\operatorname{dist}_{H}\left(\varphi\left(t_{0}, \theta_{-t_{0}} p\right) \varphi\left(t-t_{0}, \theta_{-t} p\right) D\left(\theta_{-t} p\right), A(p)\right) \\
& \leqslant \operatorname{dist}_{H}\left(\varphi\left(t_{0}, \theta_{-t_{0}} p\right) B\left(\theta_{-t_{0}} p\right), A(p)\right)<\epsilon
\end{aligned}
$$

which proves that $\hat{A}$ pullback attracts every family $D \in \mathscr{D}$.

The compactness of $A(p)$ follows since $A(p) \subset B(p)$ and $A(p)$ is closed, for every $p \in P$.

It remains to show the invariance of the family $\hat{A}$. Let $x \in A(p)$ and $t \geqslant 0$. Then there are sequences $\left\{t_{n}\right\}_{n \geqslant 0} \subset[0, \infty)$ and $\left\{x_{n}\right\}_{n \geqslant 0}$ such that $t_{n} \rightarrow \infty$ as $n \rightarrow \infty, x_{n} \in B\left(\theta_{-t_{n}} p\right)$ for every $n \geqslant 0$ and $\varphi\left(t_{n}, \theta_{-t_{n}} p\right) x_{n} \rightarrow x$ as $n \rightarrow \infty$. Using the continuity of $\varphi(t, p)$, we have that

$$
\varphi\left(t+t_{n}, \theta_{-t-t_{n}} \theta_{t} p\right) x_{n}=\varphi\left(t+t_{n}, \theta_{-t_{n}} p\right) x_{n}=\varphi(t, p) \varphi\left(t_{n}, \theta_{-t_{n}} p\right) x_{n} \rightarrow \varphi(t, p) x \text {, as } n \rightarrow \infty,
$$

which proves that $\varphi(t, p) x \in A\left(\theta_{t} p\right)$.

Now if $x \in A\left(\theta_{t} p\right)$, there exist sequences $\left\{t_{n}\right\}_{n \geqslant 0} \subset[0, \infty)$ and $\left\{x_{n}\right\}_{n \geqslant 0}$ such that $t_{n} \rightarrow \infty$ as $n \rightarrow \infty, x_{n} \in B\left(\theta_{t-t_{n}} p\right)$ for every $n \geqslant 0$ and $\varphi\left(t_{n}, \theta_{t-t_{n}} p\right) x_{n} \rightarrow x$ as $n \rightarrow \infty$. Since $t_{n} \rightarrow \infty$ as $n \rightarrow \infty$, we can assume that $t_{n} \geqslant t$ for all $n \geqslant 0$.

We have then $\varphi\left(t_{n}, \theta_{t-t_{n}} p\right) x_{n}=\varphi(t, p) \varphi\left(t_{n}-t, \theta_{t-t_{n}} p\right) x_{n}$ and since $B$ is absorbing, we can also assume that the sequence $\left\{\varphi\left(t_{n}-t, \theta_{t-t_{n}} p\right) x_{n}\right\}_{n \geqslant 0}$ is contained in $B(p)$. But $B(p)$ is compact, and we can assume that there exists $y \in B(p)$ such that $\varphi\left(t_{n}-t, \theta_{t-t_{n}} p\right) x_{n} \rightarrow y$. 
Thus $y \in A(p)$ and, by the continuity of $\varphi(t, p)$, we have $\varphi(t, p) y=x$, which concludes the invariance of $\hat{A}$ and also the theorem.

We now introduce the concepts of local attractivity and repulsion, following [38] (see also [24]).

Definition 3.2.15 (Local pullback attractivity). Let $\varphi$ be a NDS with a pullback attractor $\hat{\mathscr{A}}$. A compact invariant family $\hat{A}$ with $\hat{A} \subset \hat{A}$ is called a local pullback attractor if there exists an $\eta>0$ such that

$$
\lim _{t \rightarrow \infty} \operatorname{dist}_{H}\left(\varphi\left(t, \theta_{-t} p\right) \mathscr{O}_{\eta}\left(A\left(\theta_{-t} p\right)\right), A(p)\right)=0, \text { for all } p \in P
$$

where $\mathscr{O}_{\eta}(A(q)) \doteq\left\{x \in \mathscr{A}(q): \operatorname{dist}_{H}(x, A(q))<\eta\right\}$, for $q \in P$. The supremum of all $\eta>0$ for which the above relation holds is called local pullback radius of attraction of $A$.

Remark 3.2.16. We see that a local pullback attractor is a pullback attractor with respect to the attraction universe $\hat{\mathscr{D}}$ defined by all the families $\left\{\mathscr{O}_{\zeta}(A(p)): p \in P\right\}$ where $\zeta \in(0, \eta]$.

Definition 3.2.17 (Local backward repulsion). Let $\varphi$ be a NDS with a pullback attractor $\hat{\mathscr{A}}$. A compact invariant family $\hat{A}^{*}$ with $\hat{A}^{*} \subset \hat{A}$ is called a local repeller if there exists $\eta>0$ such that

$$
\lim _{t \rightarrow \infty} \operatorname{dist}_{H}\left(\varphi(-t, p) \mathscr{O}_{\eta}\left(A^{*}(p)\right), A^{*}\left(\theta_{-t} p\right)\right)=0, \text { for all } p \in P
$$

where $\varphi(-t, p) B:=\left[\varphi\left(t, \theta_{-t} p\right)\right]^{-1} B$ is defined as the inverse image of $B$ by the map $\varphi\left(t, \theta_{-t} p\right)$ : $\mathscr{A}\left(\theta_{-t} p\right) \rightarrow \mathscr{A}(p)$. The supremum of all $\eta>0$ such that the above relation holds is called local radius of repulsion of $A^{*}$.

Remark 3.2.18. We emphasize that the relations (3.2.4) and (3.2.5) do not hold, in general, when $\eta>0$ is the local radius of attraction and repulsion, respectively.

Theorem 3.2.19 (Existence of attractor-repeller pairs). Let $\varphi$ be a NDS with a pullback attractor $\hat{\mathscr{A}}$ and $\hat{A}^{*}$ a local repeller. Then, there exists a uniquely determined local pullback attractor $\hat{A}$, which is the maximal local pullback attractor outside $\hat{A}^{*}$ in the sense that $A(p) \cap A^{*}(p)=\varnothing$ for all $p \in P$ and any local pullback attractor $\hat{A}^{\prime}$ with $\hat{A}^{\prime} \supsetneq \hat{A}$ has nonempty intersection with $\hat{A}^{*} ;$ i.e., there exists $p \in P$ such that $A^{\prime}(p) \cap A^{*}(p) \neq \varnothing$. The pair $\left(\hat{A}, \hat{A}^{*}\right)$ is called an attractorrepeller pair. 
Proof. Since $\hat{A}^{*}$ is a local repeller, if $\eta^{*}>0$ is the local radius of repulsion of $\hat{A}^{*}$ and $\eta \in\left(0, \eta^{*}\right)$, we have that

$$
\lim _{t \rightarrow \infty} \operatorname{dist}_{H}\left(\varphi(-t, p) \mathscr{O}_{\eta}\left(A^{*}(p)\right), A^{*}\left(\theta_{-t} p\right)\right)=0, \text { for all } p \in P
$$

Consider the universe of attraction containing all the families $\hat{D}^{\zeta}$ for $\zeta \in(0, \eta]$ which are defined by

$$
D^{\zeta}(p) \doteq \mathscr{A}(p) \backslash \mathscr{O}_{\zeta}\left(A^{*}(p)\right), \text { for all } p \in P
$$

Now we will show that the family $\hat{D}^{\eta}$ is pullback absorbing with respect to $\hat{\mathscr{D}}$ (note that $\hat{D}^{\eta}$ is a compact family). Choose $\zeta \in(0, \eta]$ and $p \in P$ arbitrarily. Equation (3.2.6) gives us a $t_{0} \geqslant 0$ such that

$$
\operatorname{dist}_{H}\left(\varphi(-t, p) \mathscr{O}_{\eta}\left(A^{*}(p)\right), A^{*}\left(\theta_{-t} p\right)\right)<\frac{\zeta}{2} \text { for all } t \geqslant t_{0}
$$

which means that $\varphi(-t, p) \mathscr{O}_{\eta}\left(A^{*}(p)\right) \subseteq \mathscr{O}_{\zeta / 2}\left(A^{*}\left(\theta_{-t} p\right)\right)$ for all $t \geqslant t_{0}$. Thus, we obtain

$$
\begin{aligned}
\varphi(-t, p) D^{\eta}(p) & =\varphi(-t, p)\left(\mathscr{A}(p) \backslash \mathscr{O}_{\eta}\left(A^{*}(p)\right)\right) \\
& =\mathscr{A}\left(\theta_{-t} p\right) \backslash \varphi(-t, p) \mathscr{O}_{\eta}\left(A^{*}(p)\right) \\
& \supseteq D^{\zeta}\left(\theta_{-t} p\right), \text { for all } t \geqslant t_{0} .
\end{aligned}
$$

Applying $\varphi\left(t, \theta_{-t} p\right)$ in both sides we obtain the relation $\varphi\left(t, \theta_{-t} p\right) D^{\zeta}\left(\theta_{-t} p\right) \subseteq D^{\eta}(p)$ for all $t \leqslant t_{0}$ which proves that the family $\hat{D}^{\eta}$ is pullback absorbing with respect to $\hat{\mathscr{D}}$.

Theorem 3.2.14 guarantees the existence of a pullback attractor $\hat{A}$ with respect to $\hat{\mathscr{D}}$ with $\hat{A} \subset \hat{D}^{\eta}$. Now, since $A(p) \subseteq D^{\eta / 2}(p)$ for all $p \in P$ we have that $\mathscr{O}_{\eta / 2}(A(p)) \subseteq D^{\eta}(p)$ for all $p \in P$. But $\hat{D}^{\eta} \in \hat{\mathscr{D}}$ and since $\hat{A}$ pullback attracts $\hat{D}^{\eta}, \hat{A}$ pullback attracts $\left\{\mathscr{O}_{\eta / 2}(A(p)): p \in P\right\}$, which shows that $\hat{A}$ is a local pullback attractor.

Now, if $\tilde{\eta} \in\left[\eta, \eta^{*}\right)$ then using the same process we construct another local pullback attractor $\hat{\tilde{A}}$. But by construction and by the attraction property, we can see that $\hat{A}=\hat{\tilde{A}}$, which means that the local pullback attractor does not change if we vary $\eta \in\left(0, \eta^{*}\right)$.

If $\hat{A}^{\prime}$ is another pullback attractor with $\hat{A}^{\prime} \supsetneq \hat{A}$, there exists a $p_{0} \in P$ such that $A^{\prime}\left(p_{0}\right) \supsetneq$ $A\left(p_{0}\right)$. Let $x \in A^{\prime}\left(p_{0}\right) \backslash A\left(p_{0}\right)$ and $\sigma$. $\left(p_{0}\right)$ an entire $p_{0}$-orbit through $x$. Since $\hat{A}^{\prime}$ is a local pullback attractor and $x \in A^{\prime}\left(p_{0}\right)$ (then $\sigma_{s}\left(p_{0}\right) \in A^{\prime}\left(\theta_{s} p_{0}\right)$ for all $\left.s \in \mathbb{R}\right)$, there exists $\tilde{\eta}>0$ such that 


$$
\lim _{t \rightarrow \infty} \operatorname{dist}_{H}\left(\varphi\left(t, \theta_{-t} p_{0}\right) \mathscr{O}_{\tilde{\eta}}\left(\sigma_{-t}\left(p_{0}\right)\right), A^{\prime}\left(p_{0}\right)\right)=0
$$

Claim: If $C \subseteq \mathscr{A}\left(p_{0}\right) \backslash A\left(p_{0}\right)$ is a compact set then

$$
\lim _{t \rightarrow \infty} \operatorname{dist}_{H}\left(\varphi\left(-t, p_{0}\right) C, A^{*}\left(\theta_{-t} p_{0}\right)\right)=0
$$

Indeed, if $\epsilon \in(0, \eta)$, since $\hat{A}$ is a pullback attractor with respect to $\hat{\mathscr{D}}$ and $\hat{D}^{\epsilon} \in \hat{\mathscr{D}}$, there exists $t_{0} \leqslant 0$ such that

$$
\operatorname{dist}_{H}\left(\varphi\left(t, \theta_{-t} p_{0}\right) D^{\epsilon}\left(\theta_{-t} p_{0}\right), A\left(p_{0}\right)\right) \leq \frac{\operatorname{dist}_{H}\left(C, A\left(p_{0}\right)\right)}{2}, \text { for all } t \leqslant t_{0},
$$

which implies that $C \cap \varphi\left(t, \theta_{-t} p_{0}\right) D^{\epsilon}\left(\theta_{-t} p_{0}\right)=\varnothing$ for all $t \leqslant t_{0}$. Hence, $D^{\epsilon}\left(\theta_{-t} p_{0}\right) \cap \varphi\left(-t, p_{0}\right) C=$ $\varnothing$ for all $t \leqslant t_{0}$, which means that $\operatorname{dist}_{H}\left(\varphi\left(-t, p_{0}\right) C, A^{*}\left(\theta_{-t} p_{0}\right)\right)<\epsilon$ for all $t \leqslant t_{0}$ and proves our claim.

Now, by our claim, $\lim _{t \rightarrow \infty} \operatorname{dist}_{H}\left(\sigma_{-t}\left(p_{0}\right), A^{*}\left(\theta_{-t} p_{0}\right)\right)=0$, and so for each $n \geqslant 0$ we can find $t_{n}>n$ and $x_{n} \in A^{*}\left(\theta_{-t_{n}} p_{0}\right)$ such that $\lim _{n \rightarrow \infty} \operatorname{dist}_{H}\left(\sigma_{-t_{n}}\left(p_{0}\right), x_{n}\right)<\tilde{\eta}$. In this way, $x_{n} \in \mathscr{O}_{\tilde{\eta}}\left(\sigma\left(\theta_{-t_{n}} p_{0}\right)\right)$ and

$$
\lim _{n \rightarrow \infty} \operatorname{dist}_{H}\left(\varphi\left(t_{n}, \theta_{-t_{n}} p_{0}\right) x_{n}, A^{\prime}\left(t_{0}\right)\right)=0 \text {. }
$$

Since $x_{n} \in A^{*}\left(\theta_{-t_{n}} p_{0}\right)$ and $\hat{A}^{*}$ is invariant, the sequence $\left\{\varphi\left(t_{n}, \theta_{-t_{n}} p_{0}\right) x_{n}\right\}_{n \geqslant 0}$ is in $A^{*}\left(p_{0}\right)$. By the compactness of $A^{*}\left(p_{0}\right)$ we can assume that there exists $z \in A^{*}\left(p_{0}\right)$ such that $\varphi\left(t_{n}, \theta_{-t_{n}} p_{0}\right) x_{n} \rightarrow$ $z$ as $n \rightarrow \infty$ and then it is clear that $\operatorname{dist}_{H}\left(z, A^{\prime}\left(p_{0}\right)\right)=0$, and by the compactness of $A^{\prime}\left(p_{0}\right)$, we have that $z \in A^{\prime}\left(p_{0}\right)$, which proves that $A^{\prime}\left(p_{0}\right) \cap A^{*}\left(p_{0}\right) \neq \varnothing$ and completes the proof.

It is important to remark that the existence of a local pullback attractor does not guarantee the existence of a local forward repeller (see [38]).

Theorem 3.2.20 (Dynamics of attractor-repeller pairs). Let $\varphi$ be a NDS with a pullback attractor $\hat{\mathcal{A}}$. Let $\left(\hat{A}, \hat{A}^{*}\right)$ be an attractor-repeller pair. The following statements hold:

(i) There exists a $\beta>0$ such that

$$
\mathscr{O}_{\beta}(A(p)) \cap \mathscr{O}_{\beta}\left(A^{*}(p)\right)=\varnothing, \text { for all } p \in P .
$$


(ii) Let $p_{0} \in P$ be a fixed real number and $C \subseteq \mathscr{A}\left(p_{0}\right) \backslash A\left(p_{0}\right)$ a compact set. Then

$$
\lim _{t \rightarrow \infty} \operatorname{dist}_{H}\left(\varphi\left(-t, p_{0}\right) C, A^{*}\left(\theta_{-t} p_{0}\right)\right)=0
$$

(iii) Let $\hat{K}$ be a compact non-autonomous set with $\hat{K} \subset \hat{\mathcal{A}}$ and $\liminf _{t \rightarrow \infty} \mathrm{d}\left(K\left(\theta_{-t} p\right), A^{*}\left(\theta_{-t} p\right)\right)>$ 0. Then

$$
\lim _{t \rightarrow \infty} \operatorname{dist}_{H}\left(\varphi\left(t, \theta_{-t} p\right) K\left(\theta_{-t} p\right), A(p)\right)=0, \text { for all } p \in P
$$

Proof. Let $\eta>0$ and $\hat{\mathscr{D}}$ as in the proof of Theorem 3.2.19; i.e., $\eta$ is the local radius of repulsion of $\hat{A}^{*}$ and $\hat{\mathscr{D}}$ is the attraction universe containing all the families $\hat{D}^{\zeta}$, with $\zeta \in(0, \eta]$.

(i) It was shown in the proof of Theorem 3.2.19 that $\hat{A} \subseteq \hat{D}^{\eta}$. This assertion then follows by taking $\beta \doteq \frac{\eta}{2}$.

(ii) This is the Claim in the proof of Theorem 3.2.19,

(iii) Choose $\zeta<\min \left\{\eta, \liminf _{t \rightarrow \infty}\left(K\left(\theta_{-t} p\right), A^{*}\left(\theta_{-t} p\right)\right)\right\}$. There exists $t_{0} \geqslant 0$ such that

$$
\mathrm{d}\left(K\left(\theta_{-t} p\right), A^{*}\left(\theta_{-t} p\right)\right) \geqslant \zeta, \text { for all } t \geqslant t_{0},
$$

which implies that $K\left(\theta_{-t} p\right) \subset D^{\zeta}\left(\theta_{-t} p\right)$ for all $t \geqslant t_{0}$. This finishes the proof since $\hat{D}^{\zeta} \in$ $\hat{\mathscr{D}}$ and $\hat{A}$ is a pullback attractor with respect to $\hat{\mathscr{D}}$.

Proposition 3.2.21 (Nonuniqueness of attractor-repeller pairs). Let $\varphi$ be a NDS with a pullback attractor $\hat{A}$. Let $\hat{A}^{*}$ and $\hat{R}^{*}$ be two local repellers such that their corresponding attractors, $\hat{A}$ and $\hat{R}$ respectively, are equal; i.e., $\hat{A}=\hat{R}$. Then,

$$
\lim _{t \rightarrow \infty} \operatorname{dist}_{H}\left(A^{*}\left(\theta_{-t} p\right), R^{*}\left(\theta_{-t} p\right)\right)=0
$$

for all $p \in P$.

Proof. Arguing by contradiction, assume that there exist $p \in P$ and sequences $\left\{t_{n}\right\}_{n \geqslant 0} \subseteq$ 
$[0, \infty),\left\{x_{n}\right\}_{n \geqslant 0}$ and $\epsilon>0$ such that $t_{n} \rightarrow \infty$ as $n \rightarrow \infty, x_{n} \in A^{*}\left(\theta_{-t_{n}} p\right)$ and

$$
\operatorname{dist}_{H}\left(x_{n}, R^{*}\left(\theta_{-t_{n}} p\right)\right) \geqslant \epsilon, \text { for all } n \geqslant 0 \text {. }
$$

Applying Theorem 3.2 .20 (iii) for the attractor-repeller pair $\left(\hat{R}, \hat{R}^{*}\right)$, since

$$
\liminf _{n \rightarrow \infty} \operatorname{dist}_{H}\left(x_{n}, R^{*}\left(\theta_{-t_{n}} p\right)\right) \geqslant \epsilon>0
$$

we have that

$$
\lim _{n \rightarrow \infty} \operatorname{dist}_{H}\left(\varphi\left(t_{n}, \theta_{-t_{n}} p\right) x_{n}, R(p)\right)=0 .
$$

Since $\varphi\left(t_{n}, \theta_{-t_{n}} p\right) x_{n} \in R^{*}(p)$ for all $n \geqslant 0$ and both $A^{*}(p)$ and $A(p)=R(p)$ are compact sets, it follows that $R(p) \cap R^{*}(p) \neq \varnothing$, which is a contradiction and proves the result.

\subsection{Morse decomposition for non-autonomous dynamical sys-}

\section{tems}

The definition of a Morse decomposition via finite attractor-repeller pair sequence is basically the same as in the autonomous case.

Definition 3.3.1. Let $\varphi$ be a NDS with pullback attractor $\hat{A}$. Assume that there exists a sequence of attractor-repeller pairs $\left(\hat{A}_{i}, \hat{A}_{i}^{*}\right)$, for $i=0, \cdots, n$, satisfying

$$
\varnothing=A_{n}^{*}(p) \subsetneq A_{n-1}^{*}(p) \subsetneq \cdots \subsetneq A_{0}^{*}(p)=\mathscr{A}(p),
$$

for all $p \in P$, and also

$$
\varnothing=A_{0}(p) \subsetneq A_{1}(p) \subsetneq \cdots \subsetneq A_{n}(p)=\mathscr{A}(p),
$$

for all $p \in P$. The collection $\left\{\hat{M}_{1}, \hat{M}_{2}, \cdots, \hat{M}_{n}\right\}$ defined by

$$
M_{i}(p) \doteq A_{i}(p) \cap A_{i-1}^{*}(p), \text { for all } p \in P \text { and } i \in\{1, \cdots, n\}
$$

is called $a$ Morse decomposition. Each family $\hat{M}_{i}$ is called a Morse set. 
Note here that, unlike the autonomous case, we need to impose the condition $\varnothing=A_{0}(p) \subsetneq$ $A_{1}(p) \subsetneq \cdots \subsetneq A_{n}(p)=\mathscr{A}(p)$ on the local pullback attractors, since Proposition 3.2.21 indicates that local pullback attractors of the attractor sequence may coincide.

The definition of a Morse decomposition is a generalization of an attractor-repeller pair in the sense that, if $\left(\hat{A}, \hat{A}^{*}\right)$ is an attractor-repeller pair such that $\varnothing \subsetneq \hat{A} \subsetneq \hat{A}$, then $\left\{\hat{A}, \hat{A}^{*}\right\}$ is a Morse decomposition.

We now present a proposition that summarizes the general properties of a Morse decomposition.

Proposition 3.3.2. Let $\varphi$ be a NDS with a pullback attractor $\hat{\mathscr{A}}$. The Morse sets of a Morse decomposition $\left\{\hat{M}_{1}, \cdots, \hat{M}_{n}\right\}$ are nonempty, invariant and isolated; i.e., there exists $a>0$ such that, for $i \neq j$

$$
\mathscr{O}_{\beta}\left(M_{i}(p)\right) \cap \mathscr{O}_{\beta}\left(M_{j}(p)\right)=\varnothing, \text { for all } p \in P, \text { and } i \neq j
$$

Proof. Firstly, choose an arbitrary Morse set $\hat{M}_{i}=\hat{A}_{i} \cap \hat{A}_{i-1}^{*}$. Since $\hat{A}_{i-1} \subsetneq \hat{A}_{i}$ there exist $p_{0} \in P$ and a point $x \in A_{i}\left(p_{0}\right) \backslash A_{i-1}\left(p_{0}\right)$. But $x \in A_{i}\left(p_{0}\right)$, so we can find an entire $p_{0}$-orbit $\sigma .\left(p_{0}\right)$ through $x$ such that $\sigma_{t}\left(p_{0}\right) \in A_{i}\left(\theta_{t} p_{0}\right)$ for all $t \in \mathbb{R}$ and since $\hat{A}_{i}$ is a local pullback attractor, for $\eta>0$ being the local radius of attraction, we have that

$$
\lim _{t \rightarrow \infty} \operatorname{dist}_{H}\left(\varphi\left(t, \theta_{-t} p_{0}\right) \mathscr{O}_{\eta}\left(\sigma_{-t}\left(p_{0}\right)\right), A_{i}\left(p_{0}\right)\right)=0
$$

Since $x \notin A_{i-1}\left(p_{0}\right)$, Theorem 3.2 .20 (ii) gives that $\lim _{t \rightarrow \infty} \operatorname{dist}_{H}\left(\sigma_{-t}\left(p_{0}\right), A_{i-1}^{*}\left(\theta_{-t} p_{0}\right)\right)=0$, and given $n \in \mathbb{N}$ we can find sequences $\left\{t_{n}\right\}_{n \geqslant 0}$ with $t_{n} \rightarrow \infty$ and $\mu_{n} \in A_{i-1}^{*}\left(\theta_{-t_{n}} p_{0}\right)$ such that

$$
d_{X}\left(\sigma_{-t_{n}}\left(p_{0}\right), \mu_{n}\right) \leqslant \frac{\eta}{2}
$$

But then $\mu_{n} \in \mathscr{O}_{\eta}\left(\sigma_{-t_{n}}\left(p_{0}\right)\right)$ and if $y_{n} \doteq \varphi\left(t_{n}, \theta_{-t_{n}} p_{0}\right) \mu_{n}$ we have that

$$
\lim _{n \rightarrow \infty} \operatorname{dist}_{H}\left(y_{n}, A_{i}\left(p_{0}\right)\right)=0 .
$$

Since $A_{i-1}^{*}\left(p_{0}\right)$ and $A_{i}\left(p_{0}\right)$ are both compact, we can assume that $y_{n} \rightarrow y_{0} \in A_{i}\left(p_{0}\right) \cap A_{i-1}^{*}\left(p_{0}\right)$ we have that $M_{i}\left(p_{0}\right)=A_{i}\left(p_{0}\right) \cap A_{i-1}^{*}\left(p_{0}\right) \neq \varnothing$. 
Furthermore, $\varphi(t, p) M_{i}(p) \subset \varphi(t, p) A_{i}(p) \cap \varphi(t, p) A_{i-1}^{*}(p)=A_{i}\left(\theta_{t} p\right) \cap A_{i-1}^{*}\left(\theta_{t} p\right)=M_{i}\left(\theta_{t} p\right)$ for all $p \in P, t \geqslant 0$ and $i \in\{1, \cdots, n\}$. Now, if $x \in M_{i}\left(\theta_{t} p\right)=A_{i}\left(\theta_{t} p\right) \cap A_{i-1}^{*}\left(\theta_{t} p\right)$ and $\sigma .\left(\theta_{t} p\right)$ is a $\theta_{t} p$-orbit through $x$ then, by the invariance of $\hat{A}_{i}$ and $\hat{A}_{i-1}^{*}, \sigma \cdot\left(\theta_{t} p\right) \in \hat{M}_{i}$ and thus $\sigma_{-t}\left(\theta_{t} p\right) \in$ $A_{i}(p) \cap A_{i-1}^{*}(p)$. Therefore $x=\sigma_{0}\left(\theta_{t} p\right) \varphi(t, p) \sigma_{-t}\left(\theta_{t} p\right) \in \varphi(t, p) M_{i}(p)$.

Choose now another Morse set $M_{j}$. We can assume without loss of generality that $j>i$ and then

$$
\begin{aligned}
\hat{M}_{i} \cap \hat{M}_{j} & =\hat{A}_{i} \cap \hat{A}_{i-1}^{*} \cap \hat{A}_{j} \cap \hat{A}_{j-1}^{*} \\
& =\hat{A}_{i} \cap \hat{A}_{j} \cap \hat{A}_{i-1}^{*} \cap \hat{A}_{j-1}^{*} \\
& =\hat{A}_{i} \cap \hat{A}_{j-1}^{*} \subseteq \hat{A}_{j-1} \cap \hat{A}_{j-1}^{*} \\
& =\varnothing .
\end{aligned}
$$

Finally the isolation property is a straightforward consequence of Theorem 3.2 .20 (i).

The Morse decompositions are not uniquely defined, as in the autonomous case.

Definition 3.3.3. A Morse decomposition $\left\{\hat{M}_{1}, \cdots, \hat{M}_{n}\right\}$ is said to be finer than the Morse decomposition $\left\{\hat{N}_{1}, \cdots, \hat{N}_{m}\right\}$ if

$$
\lim _{t \rightarrow \infty} \operatorname{dist}_{H}\left(\cup_{i=1}^{n} M_{i}\left(\theta_{-t} p\right), \cup_{i=1}^{m} N_{i}\left(\theta_{-t} p\right)\right)=0
$$

for all $p \in P$.

Remark 3.3.4. Let $\left\{\hat{M}_{1}, \cdots, \hat{M}_{n}\right\}$ be a Morse decomposition given by the local repellers

$$
\varnothing=\hat{A}_{n}^{*} \subsetneq \hat{A}_{n-1}^{*} \subsetneq \cdots \subsetneq \hat{A}_{0}^{*}=\hat{\mathscr{A}}
$$

and its corresponding local pullback attractors

$$
\varnothing=\hat{A}_{0} \subsetneq \hat{A}_{1} \subsetneq \cdots \subsetneq \hat{A}_{n}=\hat{\mathscr{A}}
$$

Assume we have a new local repeller $\hat{B}^{*}$ and its corresponding local attractor $\hat{B}$ satisfying

$$
\varnothing=\hat{A}_{n}^{*} \subsetneq \hat{A}_{n-1}^{*} \subsetneq \cdots \subsetneq \hat{A}_{i}^{*} \subsetneq \hat{B}^{*} \subsetneq \hat{A}_{i-1}^{*} \subsetneq \cdots \subsetneq \hat{A}_{0}^{*}=\hat{\mathscr{A}}
$$


and

$$
\varnothing=\hat{A}_{0} \subsetneq \hat{A}_{1} \subsetneq \cdots \subsetneq \hat{A}_{i-1} \subsetneq \hat{B} \subsetneq \hat{A}_{i} \subsetneq \cdots \subsetneq \hat{A}_{n}=\hat{A}
$$

Then, the Morse decomposition $\left\{\hat{M}_{1}, \cdots, \hat{M}_{n}\right\}$ is finer than the Morse decomposition defined by the new sequence, and this is seen simply noting that $\hat{M}_{i}=\hat{A}_{i} \cap \hat{A}_{i-1}^{*} \subset \hat{B} \cap \hat{A}_{i-1}^{*}$.

To finish this section we show a result of uniqueness of the local pullback attractors in a Morse decomposition under stronger convergence hypotheses.

Proposition 3.3.5. Let $\varphi$ be a NDS with pullback attractor $\hat{\mathscr{A}}$ and $\left\{\hat{M}_{1}, \cdots, \hat{M}_{n}\right\}$ be a Morse decomposition obtained by the finite sequence of local repellers

$$
\hat{\mathscr{A}}=\hat{A}_{0}^{*} \supsetneq \hat{A}_{1}^{*} \supsetneq \cdots \supsetneq \hat{A}_{n}^{*}=\varnothing .
$$

Moreover, assume that for all $p, x \in \mathscr{A}(p)$ and $\sigma .(p)$ a $p$-orbit through $x$ there is an $i \in\{1, \cdots, n\}$ with

$$
\lim _{t \rightarrow \infty} \operatorname{dist}_{H}\left(\sigma_{-t}(p), M_{i}\left(\theta_{-t} p\right)\right)=0
$$

Then, the representation

$A_{i}(p)=\left\{x \in \mathscr{A}(p): \lim _{t \rightarrow \infty} \operatorname{dist}_{H}\left(\sigma_{-t}(p), \cup_{j=1}^{i} M_{j}\left(\theta_{-t} p\right)\right)=0\right.$, for all entire $p$-orbits $\sigma .(p)$ through $\left.x\right\}$ holds for all $i \in\{1, \cdots, n\}$; i.e., the local pullback attractors of the Morse decomposition are uniquely defined.

Proof. ( $\subseteq$ ) Let $p \in P$ fixed, $x \in A_{i}(p)$ and $\sigma .(p)$ an entire $p$-orbit through $x$. By the hypotheses, choose $j \in\{1, \cdots, n\}$ such that

$$
\lim _{t \rightarrow \infty} \operatorname{dist}_{H}\left(\sigma_{-t}(p), M_{j}\left(\theta_{-t} p\right)\right)=0
$$

Then,

$$
\lim _{t \rightarrow \infty} \operatorname{dist}_{H}\left(\sigma_{-t}(p), A_{j-1}^{*}\left(\theta_{-t} p\right)\right) \leqslant \lim _{t \rightarrow \infty} \operatorname{dist}_{H}\left(\sigma_{-t}(p), M_{j}\left(\theta_{-t} p\right)\right)=0 .
$$

Now, if $j>i$, then $\lim _{t \rightarrow \infty} \operatorname{dist}_{H}\left(\sigma_{-t}(p), A_{i}^{*}\left(\theta_{-t} p\right)\right)=0$ since $A_{j-1}^{*}(p) \subset A_{i}^{*}(p)$, which contradicts Theorem 3.2.20 (i) since $\sigma_{-t}(p) \in A_{i}\left(\theta_{-t} p\right)$ for all $t \in \mathbb{R}$. 
() Fix $p \in P$, let $x \in \mathscr{A}(p) \backslash A_{i}(p)$ and $\sigma$.(p) be an entire $p$-orbit through $x$. Then, Theorem 3.2.20 (ii) implies

$$
\lim _{t \rightarrow \infty} \operatorname{dist}_{H}\left(\sigma_{-t}(p), A_{i}^{*}\left(\theta_{-t} p\right)\right)=0
$$

If $\lim _{t \rightarrow \infty} \operatorname{dist}_{H}\left(\sigma_{-t}(p), \cup_{j=1}^{i} M_{j}\left(\theta_{-t} p\right)\right)=0$, then

$$
\lim _{t \rightarrow \infty} \operatorname{dist}_{H}\left(\sigma_{-t}(p), A_{i}\left(\theta_{-t} p\right)\right)=0
$$

since $\hat{M}_{j} \subset \hat{A}_{i}$ for $j \in\{1, \cdots, i\}$, which contradicts (3.3.1) in view of Theorem 3.2.20 (i).

\subsection{An estimate on the fractal dimension of pullback attrac-}

\section{tors}

Let us begin this section by stating an abstract result concerning the fractal dimension of a pullback attractor of a nonlinear evolution process.

Proposition 3.4.1. Let $\{T(t, s): t \geqslant s\}$ be a nonlinear evolution process with a pullback attractor $\{\mathscr{A}(t): t \in \mathbb{R}\}$. Let also $\left\{A^{*}(t): t \in \mathbb{R}\right\}$ be a local repeller in $\mathscr{A}$ and $\{A(t): t \in \mathbb{R}\}$ its corresponding local pullback attractor. Assume that the following conditions are satisfied

(a) There is a constant $L>1$ such that, for all $t \in \mathbb{R},\left.T(t, t-1)\right|_{\mathscr{A}(t-1)}$ is a Lipschitz mapping with Lipschitz constant L;

(b) There are a family $\{B(t): t \in \mathbb{R}\}$ and constants $c_{1}, c_{2} \geqslant 0$ such that $B(t)$ is a neighborhood of $A^{*}(t)$ in $\mathscr{A}(t)$ for all $t \in \mathbb{R}, \overline{B(t)} \cap A(t)=\varnothing$ and

$$
c_{1} \leqslant c(B(t)) \leqslant c_{2}, \text { for all } t \in \mathbb{R}
$$

(c) There are constants $M, \omega>0$ such that if $\{K(t): t \in \mathbb{R}\}$ is a family of compact sets with $K(t) \subset \mathscr{A}(t)$ and $K(t) \cap A^{*}(t)=\varnothing$, for all $t \in \mathbb{R}$ then

$$
\operatorname{dist}_{H}(T(t, s) K(s), A(t)) \leqslant M e^{\omega(s-t)} \text {, for all } s \leqslant t \text {. }
$$


Then, for all $t \in \mathbb{R}$, we have that

$$
c_{1} \leqslant c(\mathscr{A}(t)) \leqslant \max \left\{\frac{\omega+\ln L}{\omega} c_{2}, c(A(t))\right\}
$$

Proof. Let us fix $t \in \mathbb{R}$ and, for $n \in \mathbb{N}$, we define the compact sets

$$
K_{n} \doteq \mathscr{A}(t-n) \backslash B(t-n)
$$

and also we define subsets $\tilde{K}_{n} \subset K_{n}$ by

$$
\tilde{K}_{n} \doteq K_{n} \backslash T(t-n, t-n-1) K_{n+1}, \text { for } n \in \mathbb{N} \text {. }
$$

Clearly we have that $K_{n} \subset \mathscr{A}(t-n)$ and $K_{n} \cap A^{*}(t-n)=\varnothing$ for all $n \in \mathbb{N}$.

We also note that if $z \in \tilde{K}_{n}$ then $z \in K_{n}$, but $z \notin T(t-n, t-n-1) K_{n+1}$. However $z \in \mathscr{A}(t-$ $n)=T(t-n, t-n-1) \mathscr{A}(t-n-1)$, and $\mathscr{A}(t-n-1)=(\mathscr{A}(t-n-1) \backslash B(t-n-1)) \cup B(t-n-1)$ and hence $z \in T(t-n, t-n-1) B(t-n-1)$. Thus, $\tilde{K}_{n} \subset T(t-n, t-n-1) B(t-n-1)$.

By the precedent estimates we have that

$$
c\left(\tilde{K}_{n}\right) \leqslant c(T(t-n, t-n-1) B(t-n-1)) \leqslant c(B(t-n-1)),
$$

because $\left.T(t-n, t-n-1)\right|_{\mathscr{A}(t-n-1)}$ is a Lipschitz mapping for every $n \in \mathbb{N}$, and, in this way we obtain $c\left(\tilde{K}_{n}\right) \leqslant c_{2}$, for all $n \in \mathbb{N}$.

Now, let us define $\Omega_{n}$ by

$$
\Omega_{n} \doteq T(t, t-n) \tilde{K}_{n} \text {, for all } n \in \mathbb{N} \text {. }
$$

Also, since $\tilde{K}_{n} \subset K_{n}$, by the hypotheses (c) we have that

$$
\operatorname{dist}_{H}\left(\Omega_{n}, A(t)\right) \leqslant M e^{-\omega n} \text {, for all } n \in \mathbb{N} \text {. }
$$

Claim: It holds that $\mathscr{A}(t) \backslash B(t) \subset\left(\cup_{n \geqslant 0} \Omega_{n}\right) \cup A(t)$. 
Indeed, let $x \in \mathscr{A}(t) \backslash B(t)$. We have two possibilities for $x$ :

(i) $x \notin T(t, t-1) K_{1}$ and, in this case, $x \in \Omega_{0}=(\mathscr{A}(t) \backslash B(t)) \backslash T(t, t-1) K_{1}$;

(ii) $x \in T(t, t-1) K_{1}$ and, in this case, there is $y_{1} \in K_{1}$ such that $x=T(t, t-1) y_{1}$.

For $y_{1}$ we also have two possibilities

(iii) $y_{1} \notin T(t-1, t-2) K_{2}$ and, in this case, $y_{1} \in \tilde{K}_{1}$ and $x=T(t, t-1) y_{1} \in T(t, t-1) \tilde{K}_{1}=\Omega_{1}$;

(iv) $y_{1} \in T(t-1, t-2) K_{2}$ and, in this case, there is $y_{2} \in K_{2}$ such that $y_{1}=T(t-1, t-2) y_{2}$ and so $x=T(t, t-2) y_{2}$.

Now, applying this reasoning inductively, we obtain two possibilities for $x$ : either $x \in \Omega_{n}$ for some $n \in \mathbb{N}$ or there is a sequence $\left\{y_{n}\right\}_{n \in \mathbb{N}}$ satisfying $y_{n} \in K_{n}, x=y_{0}$ and $y_{n}=T(t-n, t-$ $n-1) y_{n+1}$ for all $n \in \mathbb{N}$ (and so $x=T(t, t-n) y_{n}$ for all $n \in \mathbb{N}$ ).

In the first possibility, clearly we have $x \in \cup_{n \geqslant 0} \Omega_{n}$. Now, if the second possibility happens, using the hypothesis (c), we have for all $n \in \mathbb{N}$ :

$$
\operatorname{dist}_{H}(x, A(t))=\operatorname{dist}_{H}\left(T(t, t-n) y_{n}, A(t)\right) \leqslant \operatorname{dist}_{H}\left(T(t, t-n) K_{n}, A(t)\right) \leqslant M e^{-\omega n},
$$

and it follows that $\operatorname{dist}_{H}(x, A(t))=0$ and, since $A(t)$ is compact, $x \in A(t)$, which concludes the proof of our claim.

We define now, for every $r>0$ and $k \geqslant 0$,

$$
N_{r, k} \doteq N\left(r / c^{k}, \tilde{K}_{k}\right)
$$

i.e., there are $x_{1}^{k}, \cdots, x_{N_{r, k}^{k}} \in \tilde{K}_{k}$ such that $\tilde{K}_{k} \subset \cup_{i=1}^{N_{r, k}} B\left(x_{i}^{k}, r / L^{k}\right)$.

In this way, if $z \in \Omega_{k}$, then there is $x \in \tilde{K}_{k}$ such that $z=T(t, t-k) x$, and there is $i \in$ $\left\{1, \cdots, N_{r, k}\right\}$ such that $\left\|x-x_{i}^{k}\right\|<r / L^{k}$. Now, if we define $\xi_{i}^{k} \doteq T(t, t-k) x_{i}^{k}$ for all $i=1, \cdots, N_{r, k}$, we have

$$
\left\|z-\xi_{i}^{k}\right\|=\left\|T(t, t-k) x-T(t, t-k) x_{i}^{k}\right\| \leqslant L^{k}\left\|x-x_{i}^{k}\right\|<r,
$$

thus $\Omega_{k} \subset \cup_{i=1}^{N_{r, k}} B\left(\xi_{i}^{k}, r\right)$ and so $N\left(r, \Omega_{k}\right) \leqslant N_{r, k}$. 
With the same arguments used in the autonomous case, namely in Proposition 3.1.4, we know that, from hypothesis (c), if $n(r) \doteq\left\lceil\frac{1}{\omega} \ln \left(\frac{M}{r}\right)\right\rceil$ and $G(r) \doteq\left(\cup_{i \geqslant n(r)+1} \Omega_{i}\right) \cup A(t)$, we have that $G(r) \subset \mathscr{O}_{r}(A(t))$ and hence $N(2 r, G(r)) \leqslant N(2 r, A(t)) \leqslant N(r, A(t))$.

If we define now $H(r) \doteq \cup_{i=0}^{n(r)} \Omega_{i}$ it follows that

$$
N(r, H(r)) \leqslant n(r) \cdot \max _{i=0, \cdots, n(r)} N\left(r \Omega_{i}\right) \leqslant n(r) \cdot \max _{i=0, \cdot, n(r)} N_{r, i}
$$

where $N_{r, i}=N\left(r / L^{i}, \tilde{K}_{i}\right)$.

From the previous claim we see that $\mathscr{A}(t)=B(t) \cup G(r / 2) \cup H(r / 2)$ for every $r>0$, and therefore

$$
\begin{aligned}
N(r, \mathscr{A}(t)) & \leqslant 3 \max \{N(r, B(t)) ; N(r, G(r / 2)) ; N(r, H(r / 2))\} \\
& \leqslant 3 \max \{N(r, B(t)) ; N(r, G(r / 2)) ; N(r / 2, H(r / 2))\} \\
& \leqslant \max \left\{N(r, B(t)) ; N(r / 2, A(t)) ; n(r / 2) \max _{i=0, \cdots, n(r / 2)} N_{r / 2, i}\right\} .
\end{aligned}
$$

Since the logarithm function is increasing we have, choosing $r>0$ small so that $\ln (1 / r)>$ 0 ,

$$
\begin{aligned}
\frac{\ln N(r, \mathscr{A}(t))}{\ln (1 / r)} \leqslant \frac{\ln 3}{\ln (1 / r)}+ & \max \left\{\frac{\ln N(r, B(t))}{\ln (1 / r)} ; \frac{\ln N(r / 2, A(t))}{\ln (1 / r)} ;\right. \\
& \left.\frac{\ln n(r / 2)}{\ln (1 / r)}+\max _{i=0, \cdots, n(r / 2)} \frac{\ln N_{r / 2, i}}{\ln (1 / r)}\right\} .
\end{aligned}
$$

We now compute the last term on the right hand side of (3.4.1):

$$
\begin{aligned}
\frac{\ln N_{r / 2, i}}{\ln (1 / r)} & =\frac{\ln N\left(r / 2 L^{i}, \tilde{K}_{i}\right)}{\ln (1 / r)} \\
& =\frac{\ln N\left(r / 2 L^{i}, \tilde{K}_{i}\right)}{\ln \left(2 L^{i} / r\right)} \cdot\left(\frac{i \ln L+\ln 2+\ln (1 / r)}{\ln (1 / r)}\right) \\
& \leqslant \frac{\ln N\left(r / 2 L^{i}, \tilde{K}_{i}\right)}{\ln \left(2 L^{i} / r\right)} \cdot\left(\frac{n(r / 2)}{\ln (1 / r)} \ln L+\frac{\ln 2}{\ln (1 / r)}+1\right),
\end{aligned}
$$

and using the calculation from Proposition 3.1.4, taking limsup for $r \rightarrow 0^{+}$in both sides of 
(3.4.1), we have that

$$
c(\mathscr{A}(t)) \leqslant \max \left\{c(B(t)), c(A(t)), \frac{\omega+\ln L}{\omega} \cdot \sup _{n \geqslant 0} c\left(\tilde{K}_{n}\right)\right\}
$$

and thus

$$
c(\mathscr{A}(t)) \leqslant \max \left\{c(A(t)), \frac{\omega+\ln L}{\omega} \cdot c_{2}\right\} .
$$

The first inequality is straightforward and we conclude the proof of this proposition.

Corollary 3.4.2. Let $\{T(t, s): t \geqslant s\}$ be a nonlinear evolution process in a Banach space $X$ with pullback attractor $\{\mathscr{A}(t): t \in \mathbb{R}\}$. Assume that the process possesses a Morse decomposition $\left\{M_{1}, \cdots, M_{n}\right\}$ given by the finite sequence of local repellers $\mathscr{A}=A_{0}^{*} \supsetneq A_{1}^{*} \supsetneq \cdots \supsetneq A_{n}^{*}=\varnothing$, and its associated local pullback attractors $\varnothing=A_{0} \subsetneq A_{1} \subsetneq \cdots \subsetneq A_{n}=\mathscr{A}$. Assume that the following conditions hold:

(a) There is a constant $L>1$ such that, for all $t \in \mathbb{R},\left.T(t, t-1)\right|_{\mathscr{A}(t-1)}$ is a Lipschitz mapping with Lipschitz constant L;

(b) For each $i \in\{1, \cdots, n\}$ there is a family $\left\{B_{i}(t): t \in \mathbb{R}\right\}$ such that $B_{i}(t)$ is a neighbourhood of $M_{i}(t)$ in $A_{i}(t)$ for all $t \in \mathbb{R}, \overline{B_{i}(t)} \cap A_{i-1}(t)=\varnothing$ and assume also that there exist constants $c_{1}, c_{2}$, independent of $i$, such that

$$
c_{1} \leqslant c\left(B_{i}(t)\right) \leqslant c_{2} \text {, for all } t \in \mathbb{R} \text { and } i \in\{1, \cdots, n\}
$$

where we set $M_{n+1}=\mathscr{A}$.

(c) There are constants $M, \omega>0$ such that if $\{K(t): t \in \mathbb{R}\}$ is a family of compact sets with $K(t) \subset A_{i}(t)$ and $K(t) \cap M_{i}(t)=\varnothing$, for all $t \in \mathbb{R}$ then

$$
\operatorname{dist}_{H}\left(T(t, s) K(s), A_{i-1}(t)\right) \leqslant M e^{\omega(s-t)}, \text { for all } s \leqslant t \text { and } i \in\{1, \cdots, n\} \text {. }
$$

Then, for all $t \in \mathbb{R}$, we have that

$$
c_{1} \leqslant c(\mathscr{A}(t)) \leqslant \frac{\omega+\ln L}{\omega} c_{2} .
$$


Proof. Firstly, on account of hypothesis (b) $i=n$, we have that there is a family $\left\{B_{n}(t): t \in \mathbb{R}\right\}$ such that $B_{n}(t)$ is a neighborhood of $M_{n}(t)=A_{n}(t) \cap A_{n-1}^{*}(t)=\mathscr{A}(t) \cap A_{n-1}^{*}(t)=A_{n-1}^{*}(t)$ in $A_{n}(t)=\mathscr{A}(t)$ for all $t \in \mathbb{R}, \overline{B_{n}(t)} \cap A_{n-1}(t)=\varnothing$, and

$$
c_{1} \leqslant c\left(B_{n}(t)\right) \leqslant c_{2} \text {, for all } t \in \mathbb{R} \text {. }
$$

Hypothesis (c), for $i=n$, implies that

$$
\operatorname{dist}_{H}\left(T(t, s) K(s), A_{n-1}(t)\right) \leqslant M e^{\omega(s-t)} \text {, for all } s \leqslant t,
$$

for every family $\{K(t): t \in \mathbb{R}\}$ of compact sets satisfying $K(t) \subset A_{n}(t)=\mathscr{A}(t)$ and $K(t) \cap$ $M_{n}(t)=K(t) \cap A_{n-1}^{*}(t)=\varnothing$ for all $t \in \mathbb{R}$. Then, we can apply Proposition 3.4.1 to obtain

$$
c_{1} \leqslant c(\mathscr{A}(t)) \leqslant \max \left\{c\left(A_{n-1}(t)\right), \frac{\omega+\ln L}{\omega} \cdot c_{2}\right\}, \text { for all } t \in \mathbb{R}
$$

Now define $\left.S(t, s) \doteq T(t, s)\right|_{A_{n-1}(s)}$ for all $s \leqslant t$. Note that the important fact in Proposition 3.4.1 is that the process is defined on a compact invariant family $\{\mathscr{A}(t): t \in \mathbb{R}\}$, and it does not matter if this family is a pullback attractor or not. Then, we can apply this proposition to the evolution process $\{S(t, s): t \geqslant s\}$ as long as we can verify the hypotheses.

To check the hypotheses we take $i=n-1$. We have the following:

(i) The pair $\left(A_{n-2}, M_{n-1}\right)$ is an attractor-repeller pair of the evolution process $\{S(t, s): t \geqslant$ $s\}$, since $A_{n-2}(t) \subset A_{n-1}(t)$ and $M_{n-1}(t)=A_{n-1}(t) \cap A_{n-2}^{*}(t)$ for all $t \in \mathbb{R}$;

(ii) $S(t, t-1)$ is a Lipschitz map with constant $L>1$ for all $t \in \mathbb{R}$;

(iii) There is a family $\left\{B_{n-1}(t): t \in \mathbb{R}\right\}$ such that $B_{n-1}(t)$ is a neighborhood of $M_{n-1}(t)$ in $A_{n-1}(t)$ for all $t \in \mathbb{R}, \overline{B_{n-1}(t)} \cap A_{n-2}(t)=\varnothing$ and

$$
c_{1} \leqslant c\left(B_{n-1}(t)\right) \leqslant c_{2} \text {, for all } t \in \mathbb{R}
$$


(iv) Hypothesis (c), for $i=n-1$, implies that

$$
\operatorname{dist}_{H}\left(T(t, s) K(s), A_{n-2}(t)\right) \leqslant M e^{\omega(s-t)} \text {, for all } s \leqslant t,
$$

for every family $\{K(t): t \in \mathbb{R}\}$ of compact sets satisfying $K(t) \subset A_{n-1}(t)=\mathscr{A}(t)$ and $K(t) \cap M_{n-1}(t)=\varnothing$ for all $t \in \mathbb{R}$.

Hence, we can apply Proposition 3.4.1 to the process $\{T(t, s): t \geqslant s\}$ defined in the compact invariant family $\left\{A_{n-1}(t): t \in \mathbb{R}\right\}$ and the attractor-repeller pair $\left(A_{n-2}, M_{n-1}\right)$ to deduce

$$
c_{1} \leqslant c\left(A_{n-1}(t)\right) \leqslant \max \left\{c\left(A_{n-2}(t)\right), \frac{\omega+\ln L}{\omega} . c_{2}\right\} \text {, for all } t \in \mathbb{R} \text {. }
$$

Joining these two results we obtain

$$
c_{1} \leqslant c(\mathscr{A}(t)) \leqslant \max \left\{c\left(A_{n-2}(t)\right), \frac{\omega+\ln L}{\omega} \cdot c_{2}\right\} \text {, for all } t \in \mathbb{R}
$$

Arguing now inductively, since $A_{0}(t)=\varnothing$ for all $t \in \mathbb{R}$, we finally arrive at

$$
c_{1} \leqslant c(\mathscr{A}(t)) \leqslant \frac{\omega+\ln L}{\omega} \cdot c_{2} \text {, for all } t \in \mathbb{R}
$$

\subsection{Example}

To illustrate our results, consider the following non-autonomous logistic equation

$$
u_{t}=u_{x x}+\lambda u-\beta(t) u^{3}
$$

for $x \in[0, \pi]$ with Dirichlet boundary conditions. We assume that there are positive constants $\beta_{1}, \beta_{2}$ such that $0<\beta_{1} \leq \beta(t) \leq \beta_{2}$ for all $t \in \mathbb{R}$. The existence of global pullback attractors for this equation is already known (see, for instance, [29]).

We consider the positive cone within $H_{0}^{1}(0, \pi)$,

$$
\mathcal{V}^{+}=\left\{u \in H_{0}^{1}(0, \pi): u(x) \geq 0 \text { for a.e. } x \in \Omega\right\}
$$


For (3.5.1), we can define an order with respect to $\mathcal{V}^{+}$. That is, $u_{0} \leq v_{0}$ if $v_{0}-u_{0} \in \mathcal{V}^{+}$.

In order to investigate further the behavior of positive solutions the following definition ([27]) is crucial.

Definition 3.5.1. A positive function with values in $C(\bar{\emptyset})$ is non-degenerate at $\infty$ (respectively $-\infty)$ if there exists $t_{0} \in \mathbb{R}$ such that $u$ is defined in $\left[t_{0}, \infty\right)$ (respectively $\left(-\infty, t_{0}\right]$ ) and there exists $a C^{1}(\bar{\Omega})$ function $\varphi_{0}(x)>0$ in $\Omega$, (vanishing on $\partial \Omega$ in case of Dirichlet boundary conditions) and satisfying $\frac{\partial \varphi_{0}}{\partial n}<0$, such that

$$
u(t, x) \geq \varphi_{0}(x) \quad \text { for all } \quad x \in \Omega \text { and all } t \geq t_{0}
$$

(respectively for all $t \leq t_{0}$ ).

From [27], we know that there exist two extremal (minimal and maximal) bounded global solutions, $\xi_{m}(\cdot)$ and $\xi_{M}(\cdot)$ for $(3.5 .1$, i.e. if $\psi(\cdot)$ is any bounded global solution for $S(t, s)$ then

$$
\xi_{m}(t) \leq \psi(t) \leq \xi_{M}(t) \text {, for all } t \in \mathbb{R} .
$$

Moreover, (3.5.1) has a pullback attractor $A(t)$ with

$$
A(t) \subset\left[\xi_{m}(t), \xi_{M}(t)\right], \text { for all } t \in \mathbb{R},
$$

with $\xi_{m}(t), \xi_{M}(t) \in A(t)$ for all $t \in \mathbb{R}$.

As a direct application of the results in [27], [28] we obtain the following description of the pullback attractor within the positive cone.

\section{Theorem 3.5.2.}

a) If $\lambda<\lambda_{1}$ then $\xi_{M}(t) \equiv 0$ for all $t \in \mathbb{R}$.

b) If $\lambda>\lambda_{1}$ then $\xi_{M}(t)$ is strictly positive and is the unique non-degenerate global solution at $-\infty$ and $+\infty$.

c) The pullback attractor for (3.5.1) in the positive cone satisfies $\mathscr{A}^{+}(t) \subset\left[0, \xi_{M}(t)\right]$. In particular, any global solution in $\mathscr{A}^{+}(t)$ is no non-degenerate at $-\infty$.

d) $\xi_{M}(t)$ pullback attracts exponentially fast every bounded set $B \subset$ int $V^{+}$. 
As the linearization around the zero solution of (3.5.1) coincides with that of the autonomous case $\beta(t)=1$, if we suppose that $\lambda \in\left(\lambda_{1}, \lambda_{2}\right)$ we know from Henry [19] that 0 is an unstable equilibria with associated unstable manifold included in the positive cone of codimension 1. From our point of view on Morse decomposition, we can conclude that 0 is a local repeller in $\mathscr{A}^{+}(t)$. Now, by Theorem 3.5.2, item d), we obtain $\xi_{M}(t)$ as the associated local attractor in the positive cone.

Thus, a direct application of Corollary 3.4.2 yields

$$
c\left(\mathscr{A}^{+}(t)\right) \leqslant \frac{\omega+\ln L}{\omega},
$$

with $\omega$ the exponential rate of attraction to $\xi_{M}(t)$ (see [27, 28] for estimation of this parameter) and $L$ the Lipschitz constant for $T\left(t, s ; u_{0}\right)=u\left(t, s ; u_{0}\right)$ with respect to the initial data $u_{0}$.

Remark 3.5.3. Observe that, since the nonlinear term is odd, if $u$ is a solution of (3.5.1) then so is $v=-u$. As a consequence, the behaviour of solutions in the positive and negative cones are symmetric and thus, if we denote by $\xi_{M}(t)$ the maximal bounded solution in the positive cone, the minimal bounded solution in the negative cone is just $-\xi_{M}(t)$, so that we get in this infinite dimensional dynamical system a similar behaviour as in Example 4.1.10. 


\section{- \\ Skew product semiflows and Morse decompositions}

Recently, the analysis of qualitative properties of evolution processes (non-autonomous dynamical systems) in general phase spaces (infinite-dimensional Banach spaces or general metric spaces) has received much attention (see, for instance, [24], [12], [15], [42], [37]). In particular, the study of pullback attractors has started to develop a wide and deep research area, providing qualitative information for the asymptotics of an increasing number of nonautonomous models of phenomena from different areas of Science as Physics, Biology, Economics, Engineering and others.

One of the drawbacks of the theory of pullback attractors is that it requires the vector field to be defined for all times in $\mathbb{R}$, and many models only consider the phenomenon after a given initial time. Of course one can artificially define the vector field for times preceding the given initial one and study the behavior of such system. But then, the pullback attractor would change for each extension and the object "pullback attractor" would loose its importance in the study of the forwards dynamics.

This approach was followed by Chepyzhov and Vishik [15] in their seminal work on the asymptotic behavior of non-autonomous problems. The crucial point here is to understand that the dynamics of a non-autonomous evolution process is (as a general rule) related to understanding the dynamics of many (possibly infinite) non-autonomous evolution processes. Once one realizes this feature, it becomes clear how rich and difficult is the subject "dynamics of non-autonomous dynamical systems". We already have some insights of that difficul- 
ties when we look at a simple concept like hyperbolicity. Indeed, in the autonomous case we choose an equilibrium, linearized it and we can compute the spectrum of the linearized operator to decide whether we have hyperbolicity or not. In the non-autonomous context, we have no way to single out which solutions will be hyperbolic and, if we were able to single out these solutions, how to verify that they actually are hyperbolic. Even the simple matter of obtaining a hyperbolic global solution from a hyperbolic equilibrium involves highly non-trivial results on the roughness of exponential dichotomies (see [11],[12]).

In this chapter we use a different approach to deal with the structure of the pullback attractor of a non-autonomous dynamical system and use it to give some estimates of their pullback attractor. To this end we begin with the study of the skew product semiflow and this study is a result of [4], which is a product of this work.

Let us now consider a general non-autonomous differential equation to illustrate the approach we will carry out in this paper. Consider the initial value problem

$$
\left\{\begin{array}{l}
\dot{x}=f(t, x), \quad t>0 \\
x(0)=x_{0} \in \mathbb{R}^{n},
\end{array}\right.
$$

where $f: \mathbb{R}^{+} \times \mathbb{R}^{n} \rightarrow \mathbb{R}^{n}$ is a "nice" function which belongs to a metric space $\mathscr{C}$. Assume that, for each $x_{0} \in \mathbb{R}^{n}$, the solution of 4.0.1) is defined for all $t \geq 0$; that is, for each $x_{0} \in \mathbb{R}^{n}$, there is a function $\mathbb{R}^{+} \ni t \mapsto x\left(t, f, x_{0}\right) \in \mathbb{R}^{n}$ satisfying (4.0.1).

Now, following [45] we define the skew product semiflow associated to 4.0.1) in the following way

$$
\begin{gathered}
\Pi(t): \mathbb{R}^{n} \times \mathscr{C} \rightarrow \mathbb{R}^{n} \times \mathscr{C} \\
\Pi(t)\left(x_{0}, f\right)=\left(x\left(t, f, x_{0}\right), f_{t}\right),
\end{gathered}
$$

where $f_{t}(s, x)=f(t+s, x)$ for all $t, s \geq 0$ and $x \in \mathbb{R}^{n}$. Suppose that the map $\mathbb{R}^{+} \times \mathscr{C} \times \mathbb{R}^{n} \ni$ $\left(t, f, x_{0}\right) \mapsto\left(x\left(t, f, x_{0}\right), f_{t}\right) \in \mathbb{R}^{n} \times \mathscr{C}$ is continuous. It is easy to see that

$$
x\left(t+s, f, x_{0}\right)=x\left(t, f_{s}, x\left(s, f, x_{0}\right)\right) .
$$

From this, it is clear that

$$
\begin{aligned}
\Pi(t+s)\left(x_{0}, f\right) & =\left(x\left(t+s, f, x_{0}\right), f_{t+s}\right)=\left(x\left(t, f_{s}, x\left(s, f, x_{0}\right)\right), f_{t}\left(f_{s}\right)\right) \\
& =\Pi(t)\left(x\left(s, f, x_{0}\right), f_{s}\right)=\Pi(t) \Pi(s)\left(x_{0}, f\right)
\end{aligned}
$$


and that $\mathbb{R}^{+} \times \mathscr{C} \times \mathbb{R}^{n} \ni\left(t, f, x_{0}\right) \mapsto \Pi(t)\left(x_{0}, f\right) \in \mathbb{R}^{n} \times \mathscr{C}$ is continuous, which means that the family $\{\Pi(t): t \geqslant 0\}$ forms a semigroup with phase space $Z=\mathbb{R}^{n} \times \mathscr{C}$.

Assume that $\{\Pi(t): t \geq 0\}$ possesses a global attractor $\mathscr{A}$ in $Z$. Then, it may seem that we have found a proper way to study the asymptotic dynamics of 4.0 .1 . In fact, the set $\mathscr{A}$ possesses dynamics associated to $\{\Pi(t): t \geq 0\}$ but it has no dynamics immediately associated to 4.0.1). An element of $\mathscr{A}$ is an element of $\mathbb{R}^{n} \times \mathscr{C}$, that is, an initial condition $y_{0} \in \mathbb{R}^{n}$ and a vector field $g$ (which is not $f$ in general) and $\Pi(t)\left(y_{0}, g\right)=\left(x\left(t, g, y_{0}\right), g_{t}\right)$ has no straightforward relation to (4.0.1). Let us try to unravel a little the connection of the points in $\mathscr{A}$ with (4.0.1).

The first step to study the dynamics of 4.0.1) is to understand the attraction property of $\{\Pi(t): t \geq 0\}$ as $t \rightarrow \infty$ relatively to the solution operator of 4.0.1).

Given a bounded subset $\tilde{B}$ of $\mathbb{R}^{n} \times \mathscr{C}$, we have that $\mathscr{A}$ attracts $\tilde{B}$ under the action of $\{\Pi(t)$ : $t \geq 0\}$ if

$$
\lim _{t \rightarrow \infty} \mathrm{d}_{H}(\Pi(t) \tilde{B}, \mathscr{A})=0 .
$$

If, for a given bounded subset $B \subset \mathbb{R}^{n}$, we only consider a bounded subset $\tilde{B}$ of the form $B \times\{f\}$, this attraction property can be written as

$$
\lim _{t \rightarrow \infty} \mathrm{d}_{H}\left(x(t, f, B) \times\left\{f_{t}\right\}, \mathscr{A}\right) \geq \lim _{t \rightarrow \infty} \mathrm{d}_{H}(x(t, f, B), A(t)),
$$

where $A(t)=\left\{x \in \mathbb{R}^{n}:\left(x, f_{t}\right) \in \mathscr{A}\right\}$ (note that $A(t) \times\left\{f_{t}\right\} \subset \mathscr{A}$ ). This means that the compact set $\mathscr{A}_{\mathbb{R}^{n}}:=\cup_{t \geqslant 0} A(t) \subset \mathbb{R}^{n}$ attracts bounded subsets of $\mathbb{R}^{n}$.

Although the set $\mathscr{A}_{\mathbb{R}^{n}}$ does not have any dynamical property with respect to the problem (4.0.1), we will see that some families in it are crucial to understand the dynamics of 44.0.1).

Essentially, note that, given a non-autonomous differential equation such as (4.0.1), we can refer to three different but closely related dynamical systems:

- The driving system $\{\Theta(t): t \geqslant 0\}$ associated to the dynamics of the time-dependent terms appearing in the equation, and which is defined by $\Theta(t) f(\cdot, x)=f_{t}(\cdot, x)=f(t+$ $\cdot, x)$

- the skew-product semiflow $\{\Pi(t): t \geqslant 0\}$ defined on the product space $Z=\mathbb{R}^{n} \times \mathscr{C}$,

- and the associated non-autonomous dynamical system $(\varphi, \Theta)_{\left(\mathbb{R}^{n}, \mathscr{C}\right)}$ with $\varphi\left(t, \Theta_{s} f\right) x_{0}=$ $x\left(t+s, f, x_{0}\right)$ (see Definition 4.1.1).

The aim of this part of the work is to describe the internal structure and dynamics (in the sense of Morse Decomposition and Lyapunov functions) of the global attractor for the skew 
product semiflow and the pullback attractor for the associated non-autonomous dynamical system. This description will be determined by the asymptotic configuration of the given driving system $\Theta(t)$. Indeed, we will show how a Morse Decomposition of the global attractor for $\Theta(t)$ produces a Morse Decomposition for the attractors both of the skew-product semiflow and the non-autonomous dynamical system. We will pay special attention to the dynamical properties inherited by this description.

\subsection{Preliminaries}

In this section we state the definitions and some known results which will be used throughout the following sections. In particular, we pay special attention to the concept of pullback attractor.

\subsubsection{Non-autonomous dynamical systems and pullback attractors}

We recall the definition of an non-autonomous dynamical system, but here we will use a more general one, where the driving system is only a semigroup rather than a group.

Definition 4.1.1. Consider two metric spaces $\left(X, d_{X}\right)$ and $\left(P, d_{P}\right)$. A non-autonomous dynamical system $(N D S)$, denoted by $(\varphi, \Theta)_{(X, P)}$, consists of two ingredients:

(i) A driving semigroup $\{\Theta(t): t \geqslant 0\}$ in $P$.

(ii) A cocycle $\varphi: \mathbb{R}^{+} \times P \times X \rightarrow X$ over $\Theta$, that is, a continuous map such that the family of mappings $\varphi(t, p): X \rightarrow X$ satisfies the cocycle property:

1) $\varphi(0, p)=I_{X}$ for all $p \in P$,

2) $\varphi(t+s, p)=\varphi(t, \Theta(s) p) \varphi(s, p)$ for all $t, s \geqslant 0$ and $p \in P$.

The associated skew product semiflow (SPSF) $\{\Pi(t): t \geq 0\} \subset \mathscr{C}(X \times P)$ is given by

$$
\Pi(t)(x, p)=(\varphi(t, p) x, \Theta(t) p)
$$

It is clear that $\{\Pi(t): t \geqslant 0\}$ is a semigroup with phase space $X \times P$. 
Remark 4.1.2. Given an $N D S(\varphi, \Theta)_{(X, P)}$ and an invariant set $R \subset P$ under the action of $\{\Theta(t)$ : $t \geqslant 0\}$, we can consider the restriction $\left.\Theta(t)\right|_{R}: R \rightarrow R$ and the restriction $\left.\varphi\right|_{\mathbb{R}^{+} \times R \times X}: \mathbb{R}^{+} \times R \times X \rightarrow$ $X$, so that we have a new NDS. In this case, the associated skew product semiflow $\left\{\left.\Pi(t)\right|_{X \times R}\right.$ : $t \geqslant 0\}$ possesses $X \times R$ as its phase space.

Definition 4.1.3. Given a global bounded solution $\eta: \mathbb{R} \rightarrow P$ of the driving $\operatorname{system}\{\Theta(t): t \geqslant$ $0\}$, a family of subsets $\hat{D}_{\eta}=\left\{D_{\eta}(t)\right\}_{t \in \mathbb{R}}$ of $X$ is called a non-autonomous $\eta$-set. If each fiber $D_{\eta}(t)$ is closed/compact/open, then $\hat{D}_{\eta}$ is called a non-autonomous closed/compact/open $\eta$-set.

Definition 4.1.4. A non-autonomous $\eta$-set $\hat{D}_{\eta}$ is said to be forward invariant under the NDS $(\varphi, \Theta)_{(X, P)}$ if $\varphi(t, \eta(s)) D_{\eta}(s) \subset D_{\eta}(t+s)$ for all $s \in \mathbb{R}$ and $t \geqslant 0$. It is said to be invariant if $\varphi(t, \eta(s)) D_{\eta}(s)=D_{\eta}(t+s)$ for all $s \in \mathbb{R}$ and $t \geqslant 0$.

Definition 4.1.5. Given two non-autonomous $\eta$-sets $\hat{D}_{\eta}$ and $\hat{A}_{\eta}$, we say that $\hat{A}_{\eta} \eta$-pullback attracts $\hat{D}_{\eta}$ if

$$
\lim _{t \rightarrow \infty} \mathrm{d}_{H}\left(\varphi(t, \eta(s-t)) D_{\eta}(s-t), A_{\eta}(s)\right)=0, \text { for each } s \in \mathbb{R}
$$

Definition 4.1.6. $A \eta$-universe $\hat{\mathscr{D}}_{\eta}$ is a collection of nonempty non-autonomous $\eta$-sets which is closed with respect to set inclusion, i.e. if $\hat{D}_{\eta}^{1} \in \hat{\mathscr{D}}_{\eta}$ and $D_{\eta}^{2}(t) \subset D_{\eta}^{1}(t)$ for all $t \in \mathbb{R}$, then $\hat{D}_{\eta}^{2} \in$ $\hat{\mathscr{D}}_{\eta}$. A non-autonomous compact $\eta$-set $\hat{S}_{\eta} \in \hat{\mathscr{D}}_{\eta}$ is called a $\hat{\mathscr{D}}_{\eta}$-pullback attractor of $(\varphi, \Theta)_{(X, P)}$ if

(i) $\hat{S}_{\eta}$ is invariant;

(ii) $\hat{S}_{\eta} \eta$-pullback attracts all families $\hat{D}_{\eta} \in \hat{\mathscr{D}}_{\eta}$.

Remark 4.1.7. The above definitions are a simple rewriting of the known definitions for the non-autonomous setting given in [12] for the case of a non-injective driving system $\{\Theta(t): t \geqslant$ $0\}$, where there may be more than one global solution through a given point $p \in P$.

Another important fact is the relationship between the global attractor of a skew product semiflow and the pullback attractors of the evolution processes it may contain. Such a relation is expressed in our next result. 
Theorem 4.1.8. Assume that the skew product semiflow $\{\Pi(t): t \geq 0\}$ possesses a global attractor $\mathscr{A}$, the driving system $\{\Theta(t): t \geq 0\}$ has a global attractor $\mathscr{A}$ and $\eta(\cdot): \mathbb{R} \rightarrow P$ is a global bounded solution for $\{\Theta(t): t \geq 0\}$. Then, the evolution process $\left\{T_{\eta}(t, s): t \geq s\right\}$ given by

$$
T_{\eta}(t, s) x=\varphi(t-s, \eta(s)) x, x \in X
$$

possesses $a \hat{\mathscr{D}}_{\eta}$-pullback attractor $\left\{\mathscr{A}_{\eta}(t): t \in \mathbb{R}\right\}$ with the property that $\mathscr{A}_{\eta}(t)=\{x \in X:(x, \eta(t)) \in$ $\mathscr{A}\}$, where $\hat{\mathscr{D}}_{\eta}$ is the collection of all non-autonomous $\eta$-sets $\hat{D}_{\eta}$ such that $\cup_{t \in \mathbb{R}} D_{\eta}(t)$ is bounded in X. Of course,

$$
\mathscr{A}=\left\{\bigcup_{t \in \mathbb{R}} \mathscr{A}_{\eta}(t) \times\{\eta(t)\}, \eta(\cdot) \text { is a global bounded solution for }\{\Pi(t): t \geq 0\}\right\}
$$

Proof: Define $K=\overline{\eta(\mathbb{R})}$, which is a compact set in $P$ and invariant under the action of $\{\Theta(t)$ : $t \geqslant 0\}$. Thus the semigroup $\left\{\Pi_{K}(t): t \geqslant 0\right\}$ given by the restriction $\Pi_{K}(t)=\left.\Pi(t)\right|_{X \times K}: X \times$ $K \rightarrow X \times K$ is well defined and has a global attractor $\mathscr{A}_{K}$. By Theorem 3.3 in [5], the nonautonomous associated set $\left\{\mathscr{A}_{K}(p)\right\}_{p \in K}$ is a pullback attractor of the NDS $(\varphi, \Theta)_{(X, K)}$, hence the family $\left\{\mathscr{A}_{\eta}(t)\right\}_{t \in \mathbb{R}}$ given by $\mathscr{A}_{\eta}(t)=\mathscr{A}_{K}(\eta(t))$, for all $t \in \mathbb{R}$, is the pullback attractor for the evolution process $\left\{T_{\eta}(t, s): t \geqslant s\right\}$. The last assertion is straightforward.

With this result, we can state the following (and its proof is a direct consequence of Theorem 3.1.5):

Theorem 4.1.9. With the hypotheses of Theorem 4.1.8, assume that the skew product semiflow $\{\Pi(t): t \geqslant 0\}$ is a generalized gradient-like semigroup with the family of invariant sets $\Psi=$ $\left\{\Xi_{1}, \ldots, \Xi_{p}\right\}$ satisfying the hypotheses of Theorem 3.1.5, then for any bounded global solution $\eta(\cdot): \mathbb{R} \rightarrow P$ of $\{\Theta(t): t \geq 0\}$ in $\mathscr{A}$, if $\left\{\mathscr{A}_{\eta}(t): t \in \mathbb{R}\right\}$ is the pullback attractor of $\left\{T_{\eta}(t, s): t \geq s\right\}$ given by $T_{\eta}(t, s) x=\varphi(t-s, \eta(s)) x, x \in X$ we have that

$$
c\left(\mathscr{A}_{\eta}(t)\right) \leqslant \frac{\omega+\ln (L)}{\omega} \max _{i=1, \ldots, p} c\left(W_{l o c}^{u}\left(\Xi_{i}\right)\right), \text { for all } t \in \mathbb{R}
$$

where $\omega, L>0$ are the constants given in Theorem 3.1 .5 . 


\subsubsection{Time dependence and translations}

We now describe the set of functions which lead to the phase space $P$ for the driving system $\Theta(t)$. For a more detailed approach, we refer to [45].

For any two Banach spaces $V, W$ we will let $C(I, W), C(V, W)$ and $C(I \times V, W)$ denote the spaces of continuous functions defined on, respectively, $I, V$ and $I \times V$, and taking values on $W$, where $I$ denotes an interval on the real line, and our primarily interest is in two cases: $I=\mathbb{R}^{+}$and $I=\mathbb{R}$. In addition to these spaces, we define $C_{b}(V, W)$ (and $C_{b}(I \times V, W)$ ) to be the collection of all $f \in C(V, W)$ (and $C(I \times V, W))$ such that for every bounded set $B \subset V$ (and every compact set $J \subset I$ ), there is a $K_{0} \geqslant 0$, such that $\|f(u)\|_{W} \leqslant K_{0}$ (and $\|f(t, u)\|_{W} \leqslant K_{0}$ ), for all $u \in B$ (and $(t, u) \in J \times B$ ).

These spaces of continuous functions are Frechét spaces with a metric topology which is described by the uniform convergence on bounded sets. The metric in this case is generated by a countable family of pseudonorms $\|\cdot\|_{k}$ as follows: Let $B_{k}$ be the closed neighborhood of the origin in $V$ of radius $k$, and set $I_{k}=I \cap[-k, k]$. Define

$$
\|f\|_{k} \doteq \sup _{u \in B_{k}, t \in I_{k}}\|f(t, u)\|_{W}
$$

A sequence $f_{n}$ is said to converge to $f$, i.e. $f=\lim _{n \rightarrow \infty} f_{n}$, whenever

$$
\lim _{n \rightarrow \infty}\left\|f-f_{n}\right\|_{k}=0, \text { for all } k \geqslant 1 .
$$

It turns out that $C_{b}(I \times V, W)$ is a complete metric space with this metric.

We now restrict our attention to the cases $I=\mathbb{R}^{+}$or $I=\mathbb{R}$. For each $f \in C(I \times V, W)$, we define the translate $f_{\tau}$ by

$$
f_{\tau}(t, u) \doteq f(t+\tau, u), \quad u \in V \text { and } t, \tau \in I
$$

Note that $f_{\tau} \in C(I \times V, W) \doteq \mathscr{C}$ for all $\tau \in I$. Furthermore, the mapping $(f, \tau) \rightarrow f_{\tau}$ is a continuous map of $\mathscr{C} \times I$ into $\mathscr{C}$, where $\mathscr{C}$ has the topology defined by the uniform convergence on compact sets. Next, we define the positive orbit and the full orbit through $f$ by

$$
\gamma^{+}(f) \doteq\left\{f_{\tau}: \tau \in \mathbb{R}^{+}\right\} \quad \text { and } \quad \gamma(f) \doteq\left\{f_{\tau}: \tau \in \mathbb{R}\right\}
$$


Similarly, the positive hull and the full hull are defined by

$$
P=H^{+}(f) \doteq{\overline{\gamma^{+}(f)}}^{\mathscr{C}} \quad \text { and } \quad P=H(f) \doteq \overline{\gamma(f)}^{\mathscr{C}}
$$

\section{Example}

In this subsection we develop an example in which we can describe the structure for the closure of a given non-autonomous function. Note that one of the motivations of our main results is to derive qualitative properties of non-autonomous dynamical systems from a detailed description of the limit points on this structure.

Example 4.1.10. Given two continuous functions $f, g: \mathbb{R}^{n} \rightarrow \mathbb{R}^{n}$, we define $r: \mathbb{R}^{+} \times \mathbb{R}^{n} \rightarrow \mathbb{R}$ by

$$
r(t, x)=h(t) f(x)+(1-h(t)) g(x),
$$

where $h: \mathbb{R}^{+} \rightarrow \mathbb{R}^{+}$is defined as follows:

Let $\left\{a_{n}\right\},\left\{b_{n}\right\},\left\{c_{n}\right\},\left\{d_{n}\right\}$ be sequences of real numbers with

(i) $a_{0}=0$;

(ii) $a_{n}<b_{n}<c_{n}<d_{n}<a_{n+1}$ for all $n \in \mathbb{N}$;

(iii) If $t_{n} \doteq b_{n}-a_{n}$ and $\tau_{n} \doteq d_{n}-c_{n}$, then $t_{n}, \tau_{n} \rightarrow \infty$ as $n \rightarrow \infty$;

(iv) If $\gamma_{n} \doteq b_{n}-c_{n}$ and $\lambda_{n} \doteq a_{n+1}-d_{n}$, then $\gamma_{n}, \lambda_{n} \rightarrow \infty$ as $n \rightarrow \infty$.

Now, we define the function $h$ in such a way that is smooth, $0 \leqslant h \leqslant 1, h(t)=1$ if $t \in\left[a_{n}, b_{n}\right]$ for some $n \in \mathbb{N}$ and $h(t)=0$ if $t \in\left[c_{n}, d_{n}\right]$ for some $n \in \mathbb{N}$.

If $\Theta(t) r(\cdot, x)=r(t+\cdot, x)$ for each $t \geqslant 0$ and $x \in X$, let $P=H^{+}(r)$ be the positive hull of $r$. Then $\{\Theta(t): t \geqslant 0\}$ is a semigroup in $P$.

First, we choose the sequence $t_{n}^{*}=a_{n}+\frac{t_{n}}{2}$, thus

$$
\Theta\left(t_{n}^{*}\right) r(s, x)=r\left(s+t_{n}^{*}, x\right)=f(x)
$$

if $s \in\left[0, \frac{t_{n}}{2}\right]$. Hence $\Theta\left(t_{n}^{*}\right) r \rightarrow f$ as $n \rightarrow \infty$ in the uniform convergence on bounded sets, which shows that $f \in \omega(r)$, the omega-limit set of $r$. Choosing $\tau_{n}^{*}=c_{n}+\frac{\tau_{n}}{2}$ we can see that $\Theta\left(\tau_{n}^{*}\right) r \rightarrow g$ as $n \rightarrow \infty$, which shows that $g \in \omega(r)$ in a similar way. 
Choosing $\gamma_{n}^{*}=b_{n}+\frac{\gamma_{n}}{2}$ we see that

$$
\Theta\left(\gamma_{n}^{*}\right) r(t, x)=r\left(t+\gamma_{n}^{*}, x\right)
$$

Using the Arzelà-Ascoli theorem and a diagonalization process, we can see that there exists a function $\xi^{*}: \mathbb{R} \times \mathbb{R}^{n} \rightarrow \mathbb{R}^{n}$ and a subsequence $\left\{\gamma_{n_{k}}^{*}\right\}$ of $\left\{\gamma_{n}^{*}\right\}$ such that $\Theta\left(\gamma_{n_{k}}^{*}\right) r \rightarrow \xi^{*}$ in the topology of uniform convergence on bounded sets. Also, since $\Theta\left(\gamma_{n}^{*}\right) r(t, x)=r\left(t+\gamma_{n}^{*}, x\right)$, we can see that if $t \in\left[-\gamma_{n}^{*}, b_{n}-\gamma_{n}^{*}\right]$, then $\Theta\left(\gamma_{n}^{*}\right) r(t, x)=f(x)$ for all $x \in \mathbb{R}^{n}$. Thus $\xi(t) \stackrel{t \rightarrow \infty}{\longrightarrow} f$, where $\xi(t)=\xi^{*}(t, \cdot)$ and, analogously, $\xi(t) \stackrel{t \rightarrow-\infty}{\longrightarrow}$ g. It is clear that $\xi$ is a global solution for the driving system $\{\Theta(t): t \geqslant 0\}$, therefore $\xi$ is a connection between $f$ and $g$.

With the same reasoning, we can construct a connection $\psi$ between $g$ and $f$. In this way we can have some description of what happens in $P=H(r)$.

In this example, we can see that, if we want a Morse decomposition for the global attractor of $\{\Theta(t): t \geqslant 0\}$, then $f$ and $g$ would be both in the same Morse set, and this will be used in Section 4.5 ,

\subsubsection{Homoclinic structures in $\omega$-limit sets}

At light of Example4.1.10, we can state a more general result concerning $\omega$-limit sets and homoclinic structures of a given gradient-like semigroup with a finite number of stationary points.

Proposition 4.1.11. Let $\{\Pi(t): t \geqslant 0\}$ a semigroup with a global attractor $\mathscr{A}$ with a finite set of stationary points $\mathscr{E}$. Let $z \in Z$ and assume that there exist $x, y \in \omega(z)$ such that $x \in \mathscr{E}$ and $x \neq y$. Then $x$ is a chain recurrent point.

Proof: It is straightforward from the definition of the $\omega$-limit set.

Proposition 4.1.12. Let $\{\Pi(t): t \geqslant 0\}$ be a semigroup with a global attractor $\mathscr{A}$, a finite set of stationary points $\mathscr{E}$ and assume that it satisfies $(G 1)$. Let $z \in Z$ and assume that there exist $x, y \in \omega(z) \cap \mathscr{E}$ with $x \neq y$. Then, there is a finite collection $\left\{\xi_{1}, \cdots, \xi_{n}\right\}$ of global solutions and points $\left\{z_{1}, \cdots, z_{n+1}\right\} \subset \mathscr{E}$, with $z_{1}=x$ and $z_{n+1}=y$ such that

$$
z_{i} \stackrel{t \rightarrow-\infty}{\longleftarrow} \xi_{i}(t) \stackrel{t \rightarrow \infty}{\longrightarrow} z_{i+1}, \text { for } i=1, \cdots, n
$$


Proof: See [11, Lemma 2.2].

This last proposition generalizes the case of Example 4.1.10 and gives us some information about the structure of the $\omega$-limit sets of gradient-like semigroups.

\subsection{Morse decomposition for a skew product semiflow}

In this section we will describe the relationships between the Morse decompositions of the skew product semiflow, the driving semiflow and the pullback attractors associated to evolution processes related to the global solutions of the driving semiflow.

Indeed, our primary interest is to obtain a Morse decomposition for the global attractor $\mathscr{A}$ of the skew product semiflow $\{\Pi(t): t \geqslant 0\}$ in terms of a Morse decomposition of the global attractor $\mathscr{A}$ of the driving system $\{\Theta(t): t \geqslant 0\}$.

\subsubsection{The lift of a Morse decomposition from $P$ to $X \times P$}

Definition 4.2.1. Given any $R \subset P$ and $\mathscr{D} \subset X \times P$, we define the subset $R^{\mathscr{D}} \subset X \times P$ by

$$
R^{\mathscr{D}}=\{(x, p) \in \mathscr{D}: p \in R\}
$$

The set $R^{\mathscr{D}}$ is called the lift of $R$ in $\mathscr{D}$.

Remark 4.2.2. If $\psi_{2}: X \times P \rightarrow P$ is the projection on the second coordinate, that is, $\psi_{2}(x, p)=p$ for all $(x, p) \in X \times P$, then we can see that $R^{\mathscr{D}}=\psi_{2}^{-1}(R) \cap \mathscr{D}$.

We can now prove the main theorem of this section:

Theorem 4.2.3. Let $(\varphi, \Theta)_{(X, P)}$ be a non-autonomous dynamical system and $\{\Pi(t): t \geqslant 0\}$ the associated skew product semiflow. Assume that $\{\Pi(t): t \geqslant 0\}$ possesses a global attractor $\mathscr{A}$ and also that the driving system $\{\Theta(t): t \geqslant 0\}$ has a global attractor $\mathscr{A}$ with a Morse decomposition $\left\{M_{1}, \cdots, M_{n}\right\}$. Assume also that $[\Theta(t)]^{-1}\left(M_{i}\right) \subset M_{i}$, for all $t \geqslant 0$ and all $i=1, \cdots, n$. Then, the family $\left\{M_{1}^{\mathscr{A}}, \cdots, M_{n}^{\mathscr{A}}\right\}$ is a Morse decomposition for the global attractor $\mathscr{A}$ of $\{\Pi(t): t \geqslant 0\}$. Moreover, the sets $M_{i}^{\mathcal{A}}$ coincide with the global attractors of the semigroups $\left\{\Pi_{i}(t): t \geqslant 0\right\}$ defined on $X \times M_{i}$ by $\Pi_{i}(t)=\left.\Pi(t)\right|_{X \times M_{i}}$ for each $i=1, \cdots, n$. 
Proof: First we prove the non-emptiness of each $M_{i}^{\mathscr{A}}$. Let $p \in M_{i}$ (since it is non-empty, such $p$ exists) and take $x \in X$. Since $\mathscr{A}$ attracts points of $X \times P$ under the action of $\{\Pi(t): t \geqslant 0\}$, we have that

$$
\lim _{t \rightarrow \infty} \mathrm{d}_{H}(\Pi(t)(x, p), \mathscr{A})=0 .
$$

Since $\mathscr{A}$ is compact and non-empty, we know that there exists a sequence $\left\{t_{n}\right\}_{n \in \mathbb{N}}$ and a point $\left(x_{0}, p_{0}\right) \in \mathscr{A}$ such that $t_{n} \rightarrow \infty$ and $\Pi\left(t_{n}\right)(x, p) \rightarrow\left(x_{0}, p_{0}\right)$. But $\Pi\left(t_{n}\right)(x, p)=\left(\varphi\left(t_{n}, p\right) x, \Theta\left(t_{n}\right) p\right)$, and thus $\varphi\left(t_{n}, p\right) x \rightarrow x_{0}$ and $\Theta\left(t_{n}\right) p \rightarrow p_{0}$. Since $M_{i}$ is closed and invariant, $p_{0} \in M_{i}$ for some $i \in\{1, \ldots, n\}$. Hence $\left(x_{0}, p_{0}\right) \in \mathscr{A}$ and $p_{0} \in M_{i}$, which shows that $\left(x_{0}, p_{0}\right) \in M_{i}^{\mathscr{A}}$.

Now we prove the invariance of each $M_{i}^{\mathscr{A}}$ under the action of $\{\Pi(t): t \geqslant 0\}$. Let $(x, p) \in$ $M_{i}^{\mathscr{A}}$. Then

$$
\Pi(t)(x, p)=(\varphi(t, p) x, \Theta(t) p)
$$

and, since $\mathscr{A}$ is invariant by $\Pi,(\varphi(t, p) x, \Theta(t) p) \in \mathscr{A}$. From the invariance of $M_{i}, \Theta(t) p \in M_{i}$ and thus $\Pi(t)(x, p) \in M_{i}^{\mathscr{A}}$. Now, if $(y, q) \in M_{i}^{\mathscr{A}}$, from the invariance of $\mathscr{A}$, there exists $(x, p) \in$ $\mathscr{A}$ such that

$$
\Pi(t)(x, p)=(y, q)
$$

which implies that $y=\varphi(t, p) x$ and $\Theta(t) p=q \in M_{i}$. Thus $p \in[\Theta(t)]^{-1}\left(M_{i}\right) \subset M_{i}$, so $p \in M_{i}$ and hence $(x, p) \in M_{i}^{\mathscr{A}}$.

Since $M_{i}^{\mathscr{A}} \subset \mathscr{A}$, to show that it is compact it remains to show that it is closed. Assume that $\left\{\left(x_{n}, p_{n}\right)\right\}_{n \in \mathbb{N}}$ is a sequence in $M_{i}^{\mathscr{A}}$ such that $\left(x_{n}, p_{n}\right) \rightarrow(x, p)$ as $n \rightarrow \infty$. Clearly $(x, p) \in \mathscr{A}$ and since $\left\{p_{n}\right\}_{n \in \mathbb{N}} \subset M_{i}, p \in M_{i}$ and thus $(x, p) \in M_{i}^{\mathscr{A}}$.

Now, if $(x, p) \in M_{i}^{\mathscr{A}} \cap M_{j}^{\mathscr{A}}$, with $i \neq j$, then it implies that $p \in M_{i} \cap M_{j}$, which is a contradiction because they are disjoint. Therefore $M_{i}^{\mathscr{A}}$ and $M_{j}^{\mathscr{A}}$ are disjoint if $i \neq j$.

Now, given a global solution $\xi: \mathbb{R} \rightarrow \mathscr{A}$ for $\{\Pi(t): t \geqslant 0\}$ we have that

$$
\xi(t)=(x(t), \eta(t)), \text { for all } t \geqslant 0,
$$

where $\eta: \mathbb{R} \rightarrow \mathscr{A}$ is a global solution for $\{\Theta(t): t \geqslant 0\}$ and $x: \mathbb{R} \rightarrow X$ satisfies $\varphi(t, \eta(s)) x(s)=$ $x(t+s)$ for all $t \geqslant 0$ and $s \in \mathbb{R}$. Since $\left\{M_{1}, \cdots, M_{n}\right\}$ is a Morse decomposition for $\mathscr{A}$, there exist $1 \leqslant i<j \leqslant n$ such that

$$
M_{j} \stackrel{t \rightarrow-\infty}{\longleftarrow} \eta(t) \stackrel{t \rightarrow \infty}{\longrightarrow} M_{i}
$$

We will show now that $\xi(t) \rightarrow M_{i}^{\mathcal{A}}$. For this purpose, assume that this is not the case, that is, 
assume that there exist $\epsilon_{0}>0$ and a sequence $\left\{t_{n}\right\}_{n \in \mathbb{N}}$ with $t_{n} \rightarrow \infty$ as $n \rightarrow \infty$ and

$$
d\left(\xi\left(t_{n}\right), M_{i}^{\mathscr{A}}\right) \geqslant \epsilon_{0} \text {, for all } n \in \mathbb{N} \text {. }
$$

But $\left\{\xi\left(t_{n}\right)\right\}_{n \in \mathbb{N}} \subset \mathscr{A}$ and we can also assume that $\xi\left(t_{n}\right) \rightarrow(x, p) \in \mathscr{A}$. Since $\eta\left(t_{n}\right) \rightarrow M_{i}$, it follows that $p \in M_{i}$ and thus $(x, p) \in M_{i}^{\mathscr{A}}$, but

$$
d\left((x, p), M_{i}^{\mathcal{A}}\right) \geqslant \epsilon_{0},
$$

and since $M_{i}^{\mathscr{A}}$ is compact, this is a contradiction.

Analogously, $\xi(t) \rightarrow M_{j}^{\mathscr{A}}$ as $t \rightarrow-\infty$. In a similar way, we can prove that there are no homoclinic structures in $\left\{M_{1}^{\mathscr{A}}, \cdots, M_{n}^{\mathscr{A}}\right\}$, as there are no homoclinic structures in $\left\{M_{1}, \cdots, M_{n}\right\}$.

To prove the second statement, fix $i=1, \cdots, n$ and let $\mathscr{A}_{i}$ be the global attractor of $\left\{\Pi_{i}(t)\right.$ : $t \geqslant 0\}$. It is easy to see that $\mathscr{A}_{i} \subset \mathscr{A}$. Now if $(x, p) \in \mathscr{A}_{i}$, then $p \in M_{i}$ and thus $(x, p) \in M_{i}^{\mathscr{A}}$. Conversely, if $(x, p) \in M_{i}^{\mathscr{A}}$, then $(x, p) \in \mathscr{A}$, and thus there exists a global solution $\xi: \mathbb{R} \rightarrow \mathscr{A}$. But, by the invariance of $M_{i}$, we have that $\xi(t) \in \mathscr{A}_{i}$ for all $t \in \mathbb{R}$, and hence $(x, p) \in \mathscr{A}_{i}$.

\subsubsection{The projection of a Morse decomposition from $X \times P$ to $P$}

We are now interested in the opposite problem. Indeed, we investigate when a given Morse decomposition on the global attractor $\mathscr{A}$ of the skew product semiflow $\{\Pi(t): t \geqslant 0\}$ generates a Morse decomposition in the global attractor $\mathscr{A}$ of the driving system $\{\Theta(t): t \geqslant$ $0\}$.

Definition 4.2.4. Given any $\mathscr{D} \subset X \times P$ and $R \subset P$, we define the subset $\mathscr{D}_{R}$ by

$$
\mathscr{D}_{R}=\{p \in R:(x, p) \in \mathscr{D} \text { for some } x \in X\} .
$$

The set $\mathscr{D}_{R}$ is called the $\mathbf{P}$-projection of $\mathscr{D}$ over $R$.

Remark 4.2.5. Notice again that if $\psi_{2}: X \times P \rightarrow P$ denotes the projection on the second coordinate, then it is clear that $\mathscr{D}_{R}=\psi_{2}(\mathscr{D}) \cap R$.

Lemma 4.2.6. Let $\mathscr{D} \subset X \times P$ be an invariant set under the action of $\{\Pi(t): t \geqslant 0\}, R \subset P$ an invariant set under the action of $\{\Theta(t): t \geqslant 0\}$ such that $[\Theta(t)]^{-1} R \subset R$ for all $t \geqslant 0$. Then, the 
projection $\mathscr{D}_{R}$ is invariant under the action of $\{\Theta(t): t \geqslant 0\}$.

Proof: Let $p \in \mathscr{D}_{R}$. Then, by definition, there exists $x \in X$ such that $(x, p) \in \mathscr{D}$ and by the invariance of $\mathscr{D}$ under the action of $\{\Pi(t): t \geqslant 0\}$, we have that, for $t \geqslant 0$,

$$
(\varphi(t, p) x, \Theta(t) p)=\Pi(t)(x, p) \in \mathscr{D}
$$

which implies that $\Theta(t) p \in \mathscr{D}_{R}$, since $\Theta(t) p \in R$ by invariance. On the other hand, given $q \in \mathscr{D}_{R}$ and $y \in X$ such that $(y, q) \in \mathscr{D}$, by the invariance again, there exists $(x, p) \in \mathscr{D}$ such that $\Pi(t)(x, p)=(y, q)$. This implies that $y=\varphi(t, p) x$ and $\Theta(t) p=q$. Thus $p \in[\Theta(t)]^{-1} R \subset R$ which proves the result.

For the following result, we will need to assume that the maps $\Theta(t): \mathscr{A} \rightarrow \mathscr{A}$ are injective, for each $t \geqslant 0$.

Theorem 4.2.7. Assume that $\left\{\mathscr{M}^{1}, \cdots, \mathscr{M}^{n}\right\}$ is a Morse decomposition for the global attractor $\mathscr{A}$ of the skew product semiflow $\{\Pi(t): t \geqslant 0\}$. Assume that the family $\left\{\mathscr{M}_{\mathscr{A}}^{1}, \cdots, \mathscr{M}_{\mathscr{A}}^{n}\right\}$ is disjoint and that $\left.\Theta(t)\right|_{\mathscr{A}}: \mathscr{A} \rightarrow \mathscr{A}$ is injective for all $t \geqslant 0$. Then, $\left\{\mathscr{M}_{\mathscr{A}}^{1}, \cdots, \mathscr{M}_{\mathscr{A}}^{n}\right\}$ is a Morse decomposition for the global attractor $\mathscr{A}$ of the driving system $\{\Theta(t): t \geqslant 0\}$.

Proof: We know that $\mathscr{M}_{\mathscr{A}}^{i}$ is compact and non-empty for each $i=1, \cdots, n$. By Lemma 4.2.6, each $\mathscr{M}_{\mathscr{A}}^{i}$ is invariant under the action of $\{\Theta(t): t \geqslant 0\}$. Now, given a global solution $\eta: \mathbb{R} \rightarrow \mathscr{A}$ of $\{\Theta(t): t \geqslant 0\}$, we choose a point $x \in X$ such that $(x, \eta(0)) \in \mathscr{A}$. Thus, there exists a global solution $\xi: \mathbb{R} \rightarrow \mathscr{A}$ through $(x, \eta(0))$, and by the injectivity of $\Theta(t)$, we have that

$$
\xi(t)=(x(t), \eta(t)), \text { for all } t \geqslant 0
$$

Since $\left\{\mathscr{M}^{1}, \cdots, \mathscr{M}^{n}\right\}$ is a Morse decomposition for $\mathscr{A}$, we have that, for some $i<j$,

$$
\mathscr{M}^{j} \stackrel{t \rightarrow-\infty}{\longleftarrow} \xi(t) \stackrel{t \rightarrow \infty}{\longrightarrow} \mathscr{M}^{i}
$$

which means that

$$
\mathscr{M}_{\mathscr{A}}^{j \stackrel{t \rightarrow-\infty}{\longleftarrow}} \eta(t) \stackrel{t \rightarrow \infty}{\longrightarrow} \mathscr{M}_{\mathscr{A}}^{i} .
$$

Again, there are no homoclinic structures in $\left\{M_{\mathscr{A}}^{1}, \cdots, M_{\mathscr{A}}^{n}\right\}$ because there are no homoclinic structures in $\left\{\mathscr{M}^{1}, \cdots, \mathscr{M}^{n}\right\}$. 


\subsection{Morse decomposition for pullback attractors}

We begin this section by stating some known results about the Morse decomposition for pullback attractors of NDS in [5], where the approach used there requires that the set $P$ be compact and that $\{\Theta(t): t \geqslant 0\}$ be in fact a group over $P$, i.e. $\Theta(t): P \rightarrow P$ is invertible, with its continuous inverse given by $\Theta(t)^{-1}=\Theta(-t)$.

Given a set $\mathscr{D} \subset X \times P$, we define the $p$-section of $\mathscr{D}$ as the set $\mathscr{D}(p) \subset X$ as

$$
\mathscr{D}(p)=\{x \in X:(x, p) \in \mathscr{D}\}
$$

In the same way, given a non-autonomous set $\hat{\mathscr{D}}=\{D(p)\}_{p \in P}$, with $D(p) \subset X$ for all $p \in P$, we define $\mathscr{D} \subset X \times P$ as

$$
\mathscr{D}=\bigcup_{p \in P} D(p) \times\{p\}
$$

Also, given $\mathscr{D} \subset X \times P$ and $R \subset P$ we define the $\mathbf{X}$-projection of $\mathscr{D}$ over $R$ by

$$
\mathscr{P}_{R}(\mathscr{D})=\underset{p \in R}{\cup} \mathscr{D}(p)
$$

In this setting, we can define the pullback attractor for the NDS $(\varphi, \Theta)_{(X, P)}$ in the following way:

Definition 4.3.1. Let $\mathscr{B}$ be the collection of all bounded sets in $X$. A non-autonomous set $\hat{S}$ is a pullback attractor of $(\varphi, \Theta)_{(X, P)}$ if:

(i) $\mathscr{P}_{P}(S) \in \mathscr{B}$ and each $S(p)$ is compact;

(ii) $\hat{S}$ is invariant under the $\operatorname{NDS}(\varphi, \Theta)_{(X, P)}$, i.e.

$$
\varphi(t, p) S(p)=S(\Theta(t) p), \text { for all } t \geqslant 0 \text { and } p \in P
$$

(iii) $\hat{S}$ pullback attracts all non-autonomous sets $\hat{D}$ such that $\mathscr{P}_{P}(D) \in \mathscr{B}$, i.e.

$$
\lim _{t \rightarrow \infty} \mathrm{d}_{H}(\varphi(t, \Theta(-t) p) D(\Theta(-t) p), S(p))=0, \text { for all } p \in P
$$


Theorem 4.3.2 (Theorem 3.3 in [5]; see also Proposition 3.31 in [24]). Assume that $\mathscr{A}$ is the global attractor of the skew product semiflow $\{\Pi(t): t \geqslant 0\}$. Then, the associated nonautonomous set $\hat{A}$ is the pullback attractor of the $\operatorname{NDS}(\varphi, \Theta)_{(X, P)}$.

Definition 4.3.3. Let $\mathscr{A}$ be the global attractor of the skew product semiflow $\{\Pi(t): t \geqslant 0\}$. A non-autonomous compact pair $(\hat{A}, \hat{R})$ is called a pullback attractor-repeller pair in $\hat{A}$ if the associated pair $(A, R)$ is an attractor-repeller of the global attractor $\mathscr{A}$ of $\Pi(t)$.

Definition 4.3.4. Let $\mathscr{A}$ be the global attractor of the skew product semiflow $\{\Pi(t): t \geqslant 0\}$. Let $\left(\hat{A}_{i}, \hat{R}_{i}\right), i=1, \cdots, n$, be pullback attractor-repeller pairs in $\hat{\mathscr{A}}$ with

$$
\varnothing=A_{0}(p) \varsubsetneqq A_{1}(p) \varsubsetneqq \cdots \varsubsetneqq A_{n}(p)=\mathscr{A}(p)
$$

and

$$
\mathscr{A}(p)=R_{0}(p) \nsupseteq R_{1}(p) \nsupseteq \cdots \nsupseteq R_{n}(p)=\varnothing
$$

for all $p \in P$. Then, the family $\hat{\mathscr{M}}=\left\{\hat{M}_{i}\right\}_{i=1}^{n}$ of invariant non-autonomous compact sets, defined by

$$
\hat{M}_{i}=\hat{A}_{i} \cap \hat{R}_{i-1}, 1 \leq i \leq n,
$$

is called a pullback Morse decomposition of $\mathscr{A}$, and each $\hat{M}_{i}$ is called pullback Morse set.

By Theorem 4.2.3 and Theorem 5.15 in [5] we can now prove the following result describing the internal asymptotic dynamics of a pullback Morse decomposition for the pullback attractor $\hat{\mathscr{A}}$ :

Theorem 4.3.5. Assume that the driving system $\{\Theta(t): t \geqslant 0\}$ has a global attractor $\mathscr{A}$ with a Morse decomposition $\left\{M_{1}, \cdots, M_{n}\right\}$. Assume also that $[\Theta(t)]^{-1}\left(M_{i}\right) \subset M_{i}$, for all $t \geqslant 0$ and all $i=1, \cdots, n$. Take the family $\left\{M_{1}^{\mathscr{A}}, \cdots, M_{n}^{\mathscr{A}}\right\}$, which is a Morse decomposition for the global attractor $\mathscr{A}$ of $\{\Pi(t): t \geqslant 0\}$. Then, the $\operatorname{NDS}(\varphi, \Theta)_{(X, \mathscr{A})}$ has a pullback Morse decomposition $\left\{\hat{M}_{1}, \cdots, \hat{M}_{n}\right\}$, where

$$
M_{i}(p)=\left\{x \in X:(x, p) \in \mathscr{M}_{i}^{\mathscr{A}}\right\}
$$

is the $p$-section of $\mathscr{M}_{i}$, for all $p \in \mathscr{A}$ and $i=1, \cdots, n$. Assume that $\hat{\mathscr{M}}$ is described by pullback attractor-repeller pairs $\left(\hat{A}_{i}, \hat{R}_{i}\right), i=1, \ldots, n$ such that $\mathscr{P}_{P}\left(A_{i}\right) \cap \mathscr{P}_{P}\left(R_{i}\right)=\varnothing$ for $i=1, \ldots, n$. 
Then, the collection of pullback Morse sets determines the limiting behavior of NDS $\varphi$ on $\hat{\mathscr{A}}$. More precisely, we have:

(i) For any singleton non-autonomous set $\hat{x}$ in $\hat{\mathcal{A}}$, by writing $\tilde{x}=\bigcup_{p \in P} x(p) \times\{p\}$, if

$$
\mathrm{d}_{H}\left(\tilde{x}, \bigcup_{i=1}^{n} \partial R_{i}\right)>0
$$

we have

$$
\lim _{t \rightarrow+\infty} \mathrm{d}_{H}\left(\varphi\left(t, \theta_{-t} p\right) x\left(\theta_{-t} p\right), \bigcup_{i=1}^{n} M_{i}(p)\right)=0
$$

(ii) If $\varphi$ is invertible on $\hat{\mathscr{A}}$ then, for any singleton non-autonomous set $\hat{x}$ in $\hat{\mathscr{A}}$ with

$$
\mathrm{d}_{H}\left(\tilde{x}, \bigcup_{i=1}^{n} \partial A_{i}\right)>0
$$

we have

$$
\lim _{t \rightarrow+\infty} \mathrm{d}_{H}\left(\varphi\left(-t, \theta_{t} p\right) x\left(\theta_{t} p\right), \bigcup_{i=1}^{n} M_{i}(p)\right)=0
$$

(iii) Moreover, under the hypotheses of Theorems 4.3.2 and 4.3.5, the family $\left\{M_{i}(p)\right\}_{p \in M_{i}}$ is the pullback attractor of the $\operatorname{NDS}(\varphi, \Theta)_{\left(X, M_{i}\right)}$. In particular, we have that

$$
\lim _{t \rightarrow \infty} \sup _{p \in M_{i}} \mathrm{~d}_{H}\left(\varphi(t, p) B, \mathscr{P}_{M_{i}}\left(M_{i}^{\mathscr{A}}\right)\right)=0
$$

for all $B \subset X$ bounded.

We can now state the main new result of this section, concerning the asymptotic behavior of the solutions for the NDS $(\varphi, \Theta)_{(X, P)}$ as $t \rightarrow \infty$ and for any $p \in P$.

Theorem 4.3.6. Let $(\varphi, \Theta)_{(X, P)}$ be a non-autonomous dynamical system and $\{\Pi(t): t \geqslant 0\}$ the associated skew product semiflow. Assume that $\{\Pi(t): t \geqslant 0\}$ possesses a global attractor $\mathscr{A}$ and also that the driving system $\{\Theta(t): t \geqslant 0\}$ has a global attractor $\mathscr{A}$ with a Morse decomposition $\left\{M_{1}, \cdots, M_{n}\right\}$. Assume that $[\Theta(t)]^{-1}\left(M_{i}\right) \subset M_{i}$, for all $t \geqslant 0$ and all $i=1, \cdots, n$. Let $\left\{M_{1}^{\mathscr{A}}, \cdots, M_{n}^{\mathscr{A}}\right\}$ be the corresponding Morse decomposition for the global attractor $\mathscr{A}$ of 
$\{\Pi(t): t \geqslant 0\}$. Then, given $(x, p) \in X \times P$, there exists $i=1, \cdots, n$ such that

$$
\lim _{t \rightarrow \infty} \mathrm{d}_{H}\left(\varphi(t, p) x, \mathscr{P}_{M_{i}}\left(M_{i}^{\mathscr{A}}\right)\right)=0
$$

Proof: Let $(x, p) \in X \times P$, and consider the solution $\xi: \mathbb{R}^{+} \rightarrow X \times P$ for the skew product semiflow $\{\Pi(t): t \geqslant 0\}$ through $(x, p)$. We know that

$$
\xi(t)=\Pi(x, p)=(\varphi(t, p) x, \Theta(t) p) \text {, for all } t \geqslant 0 .
$$

Since $\left\{M_{1}, \cdots, M_{n}\right\}$ is a Morse decomposition for the global attractor $\mathscr{A}$ of the driving system $\{\Theta(t): t \geqslant 0\}$, there exists $i=1, \cdots, n$ such that $\Theta(t) p \rightarrow M_{i}$ as $t \rightarrow \infty$. We claim that $\xi(t) \rightarrow$ $M_{i}^{\mathscr{A}}$. Assume, by contradiction, that this is not the case, i.e. there exist $\epsilon_{0}>0$ and a sequence $\left\{t_{n}\right\}_{n \in \mathbb{N}}$ such that $t_{n} \rightarrow \infty$ as $n \rightarrow \infty$ and

$$
d\left(\xi\left(t_{n}\right), M_{i}^{\mathscr{A}}\right) \geqslant \epsilon_{0} \text {, for all } n \in \mathbb{N} \text {. }
$$

But $d\left(\xi\left(t_{n}\right), \mathscr{A}\right) \stackrel{n \rightarrow \infty}{\longrightarrow} 0$ and we can assume that there exists $\left(x_{0}, p_{0}\right) \in \mathscr{A}$ such that $\xi\left(t_{n}\right) \rightarrow$ $\left(x_{0}, p_{0}\right)$. But this implies that $p_{0} \in M_{i}$, which in turn implies that $\left(x_{0}, p_{0}\right) \in M_{i}^{\mathscr{A}}$ and gives a contradiction, since

$$
0=d\left(\left(x_{0}, p_{0}\right), M_{i}^{\mathscr{A}}\right) \stackrel{n \rightarrow \infty}{\longleftarrow} d\left(\xi\left(t_{n}\right), M_{i}^{\mathscr{A}}\right) \geqslant \epsilon_{0}>0 .
$$

Thus, $\xi(t) \rightarrow M_{i}^{\mathscr{A}}$, and then $\varphi(t, p) x \rightarrow \mathscr{P}_{M_{i}}\left(M_{i}^{\mathscr{A}}\right)$.

Our last result in this section provides the equivalence between the existence of Morse decomposition and Lyapunov function for the skew product semiflow $\{\Pi(t): t \geq 0\}$ from the existence of a Lyapunov function (and thus a Morse decomposition) for the global attractor of the driving system $\{\Theta(t): t \geq 0\}$.

Theorem 4.3.7. Let $X$ and $P$ be metric spaces, $\varphi: \mathbb{R}^{+} \times P \times X \rightarrow X$ be a cocycle, $\Theta: \mathbb{R}^{+} \times X \rightarrow X$ be the driving system with global attractor $\mathscr{A}$ and $\Pi: \mathbb{R}^{+} \times X \times P \rightarrow X \times P$ be the associated skew product semiflow. Assume that $\Pi$ possesses a global attractor $\mathscr{A}$ and that $\{\Theta(t): t \geqslant 0\}$ has a global attractor $\mathscr{A}$. If there is a Lyapunov function for $\{\Theta(t): t \geqslant 0\}$, then there is a non-autonomous Morse decomposition $\mathscr{M}^{\mathscr{A}}=\left\{M_{i}^{\mathscr{A}}\right\}_{i=1}^{n}$ and a continuous Lyapunov function $L: X \times P \rightarrow \mathbb{R}^{+}$with the following properties: 
(i) $L(\varphi(t, p) x, \Theta(t) p) \leqslant L(x, p)$ for any $(x, p) \in X \times P$ and $t \geqslant 0$.

(ii) $L(\varphi(t, p) x, \Theta(t) p)=L(x, p)$ when $x \in \cup_{i=1}^{n} M_{i}(p)$ for all $t \geqslant 0$, and L takes different constant value on different Morse sets.

(iii) $L(\varphi(t, p) x, \Theta(t) p)<L(x, p)$ when $x \in X \backslash \cup_{i=1}^{n} M_{i}(p)$ for all $t>0$.

Proof: The Lyapunov function for $\{\Theta(t): t \geqslant 0\}$ generates a Morse decomposition for its global attractor $\mathscr{A}$. This Morse decomposition generates a Morse decomposition for the global attractor $\mathscr{A}$ in the phase space $X \times P$. So the result follows from [1, Theorem 3.4 and Proposition 3.5].

With these results, returning to the problem of the estimate of the fractal dimension, we can state the following

Theorem 4.3.8. Let $X$ and $P$ be metric spaces, $\varphi: \mathbb{R}^{+} \times P \times X \rightarrow X$ be a cocycle, $\Theta: \mathbb{R}^{+} \times X \rightarrow$ $X$ be the driving system with global attractor $\mathscr{A}$, a Morse decomposition $\left\{M_{1}, \cdots, M_{n}\right\}$ and $\Pi: \mathbb{R}^{+} \times X \times P \rightarrow X \times P$ be the associated skew product semiflow with global attractor $\mathscr{A}$. Let $\eta: \mathbb{R} \rightarrow \mathscr{A}$ be a bounded global solution for $\{\Theta(t): t \geqslant 0\}$ and $\{T(t, s): t \geqslant s\}$ the evolution process defined by $T(t, s)=\varphi(t-s, \eta(s))$. We know that $\{T(t, s): t \geqslant s\}$ has a pullback attractor $\{\mathscr{A}(t): t \in \mathbb{R}\}$ with a pullback Morse decomposition given by $\mathscr{M}^{\mathscr{A}}(t)=\left\{M_{i}^{\mathscr{A}}(t)\right\}_{i=1}^{n}$, for $t \in \mathbb{R}$. Assume that $\{T(t, s): t \geqslant s\}$ satisfies conditions (a), (b) and (c) of Corollary 3.4.2. Then

$$
c_{1} \leqslant c(\mathscr{A}(t)) \leqslant \frac{\omega+\ln L}{\omega} c_{2} .
$$

\subsection{Small non-autonomous perturbations}

We can see also how these structures behave under small non-autonomous perturbations. The results below follow from [11]:

Theorem 4.4.1. Let $X$ and $P$ be metric spaces $\varphi: \mathbb{R}^{+} \times P \times X \rightarrow X$ be a cocycle, $\Theta: \mathbb{R}^{+} \times X \rightarrow X$ be the driving system and $\Pi: \mathbb{R}^{+} \times X \times P \rightarrow X \times P$ be the associated skew product semiflow. Assume that $\Pi$ has a global attractor $\mathscr{A},\{\Theta(t): t \geqslant 0\}$ has a global attractor $\mathscr{A}$ and that $p_{0} \in \mathscr{A}$ is a fixed point of $\Theta$. If $\mathscr{A}(p)=\{x \in X:(x, p) \in \mathscr{A}\}$, assume that the following conditions hold: 
(a) There exists $\delta>0$ such that $\bigcup_{\left(p, p_{0}\right) \leq \delta} \mathscr{A}(p)$ is precompact.

(b) $\left\{\varphi\left(t, p_{0}\right): t \geq 0\right\}$ is a gradient-like semigroup relatively to the set of equilibria $\mathscr{E}_{0}=\left\{y_{1,0}^{*}, \cdots, y_{n, 0}^{*}\right\}$.

(c) For each $p$ sufficiently close to $p_{0}$, there exists a global solution $\eta_{p}: \mathbb{R}^{+} \rightarrow P$ of $\Theta$ through $p$ such that $\left\{\varphi\left(t-s, \eta_{p}(s)\right): t \geq s\right\}$ possesses $n$ isolated global solutions $\xi_{i, p}^{\eta, *}: \mathbb{R} \rightarrow X i=$ $1,2, \cdots, n$, and $\sup _{1 \leq i \leq n} \sup _{t \in \mathbb{R}} \mathbf{d}\left(\xi_{i, p}^{\eta_{p, *}}(t), y_{i, 0}^{*}\right) \stackrel{p \rightarrow p_{0}}{\longrightarrow} 0$.

(d) For each compact set $K \subset \mathbb{R}^{+} \times X$ and the global solution $\eta_{p}: \mathbb{R}^{+} \rightarrow P$ of $\Theta$ through $p$,

$$
\sup _{s \in \mathbb{R}} \sup _{(t, x) \in K} \mathbf{d}\left(\varphi\left(t-s, \eta_{p}(s)\right) x, \varphi\left(t-s, p_{0}\right) x\right) \stackrel{p \rightarrow p_{0}}{\longrightarrow} 0 .
$$

(e) There exist $\mu>0$ such that, if $\xi_{p}: \mathbb{R} \rightarrow X$ is a bounded solution of $\left\{\varphi\left(t-s, \eta_{p}(s)\right)\right.$ : $t \geq s\}$ with $p$ sufficiently close to $p_{0}$ so that, if there are $t_{0} \in \mathbb{R}$ and $i \in\{1, \cdots, n\}$ with $\sup _{t \leq t_{0}} \operatorname{dist}\left(\xi_{p}(t), \xi_{i, p}^{\eta_{p, *}}(\mathbb{R})\right)<\mu\left(\right.$ resp. $\left.\sup _{t \geq t_{0}} \operatorname{dist}\left(\xi_{p}(t), \xi_{i, p}^{\eta_{p, *}}(\mathbb{R})\right)<\mu\right)$, then $\lim _{t \rightarrow-\infty} \mathbf{d}\left(\xi_{p}(t), \xi_{i, p}^{\eta_{p, *}}(t)\right)=$ $0\left(\right.$ resp. $\left.\lim _{t \rightarrow \infty} \mathbf{d}\left(\xi_{p}(t), \xi_{i, p}^{\eta_{p}, *}(t)\right)=0\right)$.

Then, for all $p$ sufficiently close to $p_{0},\left\{\varphi\left(t-s, \eta_{p}(s)\right): t \geq s\right\}$ is a non-autonomous gradientlike evolution process with respect to the disjoint set of isolated invariant families $\mathscr{E}_{p}^{\eta_{p}}=\left\{\xi_{1, p}^{\eta_{p}, *}, \cdots, \xi_{n, p}^{\eta_{p}, *}\right\}$.

Theorem 4.4.2. Let $X$ and $P$ be metric spaces $\varphi: \mathbb{R}^{+} \times P \times X \rightarrow X$ be a cocycle, $\Theta: \mathbb{R}^{+} \times X \rightarrow X$ be the driving system and $\Pi: \mathbb{R}^{+} \times X \times P \rightarrow X \times P$ be the associated skew product semiflow. Assume that $\Pi$ has a global attractor $\mathscr{A},\{\Theta(t): t \geqslant 0\}$ has a global attractor $\mathscr{A}$ and $p_{0} \in \mathscr{A}$ is a fixed point of $\Theta$. If $\mathscr{A}(p)=\{x \in X:(x, p) \in \mathscr{A}\}$, assume that the following conditions hold:

a) There exists $\delta>0$ such that $\underset{d\left(p, p_{0}\right) \leq \delta}{\cup} \mathscr{A}(p)$ is precompact.

b) $\left\{\varphi\left(t, p_{0}\right): t \geq 0\right\}$ is a generalized gradient-like semigroup with isolated invariant sets $\left\{\Gamma_{1,0}^{*}, \cdots \Gamma_{n, 0}^{*}\right\}$.

c) For each $p$ sufficiently close to $p_{0}$, there exists a global solution $\eta_{p}: \mathbb{R}^{+} \rightarrow P$ of $\Theta$ through $p$ such that $\left\{\varphi\left(t-s, \eta_{p}(s)\right): t \geq s\right\}$ possesses $n \in \mathbb{N}$ isolated invariant families

$$
\Psi_{p}^{\eta_{p}}=\left\{\Xi_{1, p}^{\eta_{p}, *}(\cdot), \cdots, \Xi_{n, p}^{\left.\eta_{p, *}(\cdot)\right\}},\right.
$$


with traces $\left\{\Gamma_{1, p}^{\eta_{p}, *}, \cdots, \Gamma_{n, p}^{\eta_{p, *}}\right\}$, which behave upper e lower semi-continuously as $p$ goes to $p_{0}\left(\sup _{1 \leqslant i \leqslant n}\left[\operatorname{dist}\left(\Gamma_{i, p}^{\eta_{p}, *}, \Gamma_{i, 0}^{*}\right)+\operatorname{dist}\left(\Gamma_{i, 0}^{*}, \Gamma_{i, p}^{\eta_{p}, *}\right)\right] \stackrel{p \rightarrow p_{0}}{\longrightarrow} 0\right)$.

d) For each compact set $K \subset \mathbb{R}^{+} \times X$ and the global solution $\eta_{p}: \mathbb{R}^{+} \rightarrow P$ of $\Theta$ through $p$,

$$
\sup _{s \in \mathbb{R}} \sup _{(t, x) \in K} \mathbf{d}\left(\varphi\left(t-s, \eta_{p}(s)\right) x, \varphi\left(t-s, p_{0}\right) x\right) \stackrel{p \rightarrow p_{0}}{\longrightarrow} 0 .
$$

e) There exist $\mu>0$ such that, if $\xi_{p}: \mathbb{R} \rightarrow X$ is a bounded solution of $\left\{\varphi\left(t-s, \eta_{p}(s)\right)\right.$ : $t \geq s\}$ with $p$ sufficiently close to $p_{0}$ so that, if there are $t_{0} \in \mathbb{R}$ and $i \in\{1, \cdots, n\}$ with $\sup _{t \leq t_{0}} \operatorname{dist}\left(\xi_{p}(t), \Gamma_{i, p}^{\eta_{p}, *}\right)<\mu\left(\right.$ resp. $\left.\sup _{t \geq t_{0}} \operatorname{dist}\left(\xi_{p}(t), \Gamma_{i, p}^{\eta_{p}, *}\right)<\mu\right)$, then $\lim _{t \rightarrow-\infty} \mathbf{d}\left(\xi_{p}(t), \Xi_{i, p}^{\eta_{p}, *}(t)\right)=$ $0\left(\right.$ resp. $\left.\lim _{t \rightarrow \infty} \mathbf{d}\left(\xi_{p}(t), \Xi_{i, p}^{\eta_{p}, *}(t)\right)=0\right)$.

Then, for all $p$ sufficiently close to $p_{0},\left\{\varphi\left(t-s, \eta_{p}(s)\right): t \geq s\right\}$ is a generalized gradient-like evolution process with isolated invariant families $\Psi_{p}^{\eta_{p}}=\left\{\Xi_{1, p}^{\eta_{p}, *}(\cdot), \cdots, \Xi_{n, p}^{\eta_{p}, *}(\cdot)\right\}$.

\subsubsection{Asymptotically autonomous evolution processes}

In this section we will consider asymptotically autonomous evolution processes. Loosely speaking, an evolution process is asymptotically autonomous if it is very close to an autonomous evolution processes when the initial times are very large. This idea leads to the following definition (for a similar definition see [32]).

Definition 4.4.3. Let $\{S(t, s): t \geqslant s\}$ be an evolution process and $\left\{S_{0}(t): t \geqslant 0\right\}$ be a semigroup in a metric space $Z$. We say that

$\star\{S(t, s): t \geqslant s\}$ is asymptotically autonomous at $-\infty$ if

$$
S(t+s, s) u_{0} \stackrel{s \rightarrow-\infty}{\longrightarrow} S_{0}(t) u_{0}
$$

$\star\{S(t, s): t \geqslant s\}$ is asymptotically autonomous at $+\infty$ if

$$
S(t+s, s) u_{0} \stackrel{s \rightarrow+\infty}{\longrightarrow} S_{0}(t) u_{0}
$$

uniformly for $t$ in bounded intervals of $[0, \infty)$ and for $u_{0}$ in compact subsets of $Z$. 
In order to obtain information about an asymptotically autonomous evolution process using the results of the previous sections it is convenient to introduce a new evolution process which is close, for all initial times, to an autonomous evolution process.

Let $\tau \in \mathbb{R},\{S(t, s): t \geqslant s\}$ be an evolution process and $\{T(t): t \geqslant 0\}$ be a semigroup, and construct the following truncated evolution processes:

$\star$ Forward truncation at time $\tau$

$$
S_{\tau}(t, s)=\left\{\begin{array}{l}
S(t, s), \text { if } s \leqslant t \leqslant \tau \\
T(t-\tau) S(\tau, s), \text { if } s \leqslant \tau \leqslant t \\
T(t-s), \text { if } \tau \leqslant s \leqslant t
\end{array}\right.
$$

$\star$ Backward truncation at time $\tau$

$$
S_{\tau}(t, s)=\left\{\begin{array}{l}
T(t-s), \text { if } s \leqslant t \leqslant \tau \\
S(t, \tau) T(\tau-s), \text { if } s \leqslant \tau \leqslant t \\
S(t, s), \text { if } \tau \leqslant s \leqslant t
\end{array}\right.
$$

We have the following property for the truncations:

Theorem 4.4.4. If $\{S(t, s): t \geqslant s\}$ is an asymptotically autonomous evolution process at $-\infty$ $(a t+\infty)$ and $\left\{S_{0}(t): t \geqslant 0\right\}$ is the associated semigroup. Assume that the semigroup satisfies a uniform continuity condition, i.e. given $\epsilon>0$, a bounded interval $I \subset \mathbb{R}^{+}$and a compact set $K \subset Z$ there exists $\delta=\delta(\epsilon, I, K)$ such that $\left\|S_{0}(t) u-S_{0}(t) v\right\|<\epsilon$, if $\|u-v\|<\delta, u, v \in K$, for all $t \in I$. Then the forward (backward) truncation of $\{S(t, s): t \geqslant s\}$ satisfies $\| S_{\tau}(t+r, r) u-S_{0}(t+$ $r, r) u \|_{Z} \longrightarrow 0$ as $\tau \rightarrow-\infty(\tau \rightarrow+\infty)$ uniformly for $r \in \mathbb{R}$ and for $(t, u)$ in compact subsets of $\mathbb{R}^{+} \times Z$.

Proof: We first deal with the case of the forward truncation evolution process. We know that for each $\tau \in \mathbb{R}$, the forward truncation at $\tau$ is given by

$$
S_{\tau}(t, s)=\left\{\begin{array}{l}
S(t, s), \text { if } s \leqslant t \leqslant \tau, \\
S_{0}(t-\tau) S(\tau, s), \text { if } s \leqslant \tau \leqslant t \\
S_{0}(t-s), \text { if } \tau \leqslant s \leqslant t .
\end{array}\right.
$$


Now given $\epsilon>0$, a bounded interval $I \subset \mathbb{R}^{+}$and a compact set $K \subset Z$, from the uniform continuity of $\left\{S_{0}(t): t \geqslant 0\right\}$, there exists $0<\delta<\epsilon$ such that

$$
\left\|S_{0}(t) u-S_{0}(t) v\right\|_{Z}<\epsilon \text {, if }\|u-v\|_{Z}<\delta, u, v \in K \text {, for all } t \in J
$$

where $J=[0,2 M]$ and $M=\sup I$.

Now, since the process $\{S(t, s): t \geqslant s\}$ is asymptotically autonomous at $-\infty$, for the $\delta>0$ above, there exists $r_{0}<0$ such that, if $r \leqslant r_{0}$,

$$
\left\|S(t+r, r) u-S_{0}(t) u\right\|_{Z}<\delta \text {, for all } t \in I \text { and } u \in K \text {. }
$$

Now we have for $\tau \leqslant r_{0}$,

$$
S_{\tau}(t+r, r) u-S_{0}(t) u=\left\{\begin{array}{l}
S(t+r, r) u-S_{0}(t) u, \text { if } t+r \leqslant \tau, \\
S_{0}(t+r-\tau) S(\tau, r) u-S_{0}(t+r-\tau) S_{0}(\tau-r) u, \text { if } r \leqslant \tau \leqslant t+r, \\
0, \text { if } \tau \leqslant r,
\end{array}\right.
$$

which implies 1 that

$$
\sup _{r \in \mathbb{R}}\left\|S_{\tau}(t+r, r) u-S_{0}(t) u\right\|_{Z}<\epsilon \text {, for all } t \in I \text { and } u \in K \text {. }
$$

The case for asymptotically autonomous evolution process at $+\infty$ follows analogously, just reminding that for each bounded interval $I \subset \mathbb{R}^{+}$and each compact set $K \subset Z$, the set

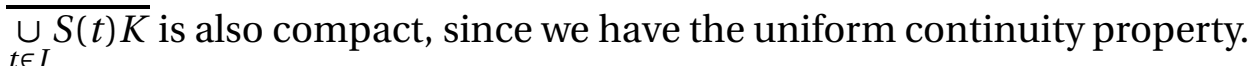

We can state the following result:

Theorem 4.4.5. Let $X$ and $P$ be a metric spaces $\varphi: \mathbb{R}^{+} \times P \times X \rightarrow X$ be a cocycle, $\Theta: \mathbb{R}^{+} \times X \rightarrow X$ be the driving system and $\Pi: \mathbb{R}^{+} \times X \times P \rightarrow X \times P$ be the associated skew product semiflow. Assume that $\Pi$ has a global attractor $\mathscr{A},\{\Theta(t): t \geqslant 0\}$ has a global attractor $\mathscr{A}$ and that $p_{0} \in \mathscr{A}$ is a fixed point of $\Theta$. Assume also that there exists a bounded global solution $\eta: \mathbb{R} \rightarrow \mathscr{A}$ such that $\eta(s) \rightarrow p_{0}$ as $s \rightarrow-\infty$ and that

a) There exists $s_{0}<0$ such that $\bigcup_{s \leqslant s_{0}} \mathscr{A}(\eta(s))$ is precompact.

\footnotetext{
${ }^{1}$ Here, for the estimate of the term $\left\|S_{0}(t+r-\tau) S(\tau, r) u-S_{0}(t+r-\tau) S_{0}(\tau-r) u\right\|_{Z}$, is where we have the need to use the interval $J$ for the uniform continuity property, since $t+r-\tau$ does not need to be in $I$, but $t+r-\tau \in J$.
} 
$\left.b^{\prime}\right)$ If $T(t):=\varphi\left(t, p_{0}\right)$, for all $t \geqslant 0$ then $\{T(t): t \geqslant 0\}$ is a generalized gradient-like semigroup with isolated invariant sets $\left\{\Gamma_{1,0}^{*}, \cdots \Gamma_{n, 0}^{*}\right\}$.

$\left.c^{\prime}\right)$ If $S(t, s):=\varphi(t-s, \eta(s))$ for all $t \geqslant s$, the evolution process $\{\varphi(t-s, \eta(s)): t \geq s\}$ possesses $n \in \mathbb{N}$ isolated invariant families

$$
\Psi=\left\{\Xi_{1}(\cdot), \cdots, \Xi_{n}(\cdot)\right\}
$$

which behave upper e lower semi-continuously as $s \rightarrow-\infty$, that is

$$
\sup _{1 \leqslant i \leqslant n}\left[d_{H}\left(\Xi_{i}^{*}(s), \Gamma_{i, 0}^{*}\right)+d_{H}\left(\Gamma_{i, 0}^{*}, \Xi_{i}(s)\right)\right] \stackrel{s \rightarrow-\infty}{\longrightarrow} 0 .
$$

$\left.d^{\prime}\right)$ There exist $\mu>0$ such that, if $\xi: \mathbb{R} \rightarrow X$ is a bounded solution of $\{\varphi(t-s, \eta(s)): t \geq s\}$ so that, if there are $t_{0} \in \mathbb{R}$ and $i \in\{1, \cdots, n\}$ with $\sup _{t \leq t_{0}} \mathbf{d}\left(\xi(t), \Gamma_{i}^{*}\right)<\mu$, then $\lim _{t \rightarrow-\infty} \mathbf{d}\left(\xi(t), \Xi_{i}^{*}(t)\right)=$ 0 .

Then, there exists $\tau_{0}<0$ such that, for all $\tau \leqslant \tau_{0}$, the forward truncated evolution process $\left\{S_{\tau}(t, s): t \geqslant s\right\}$ is a generalized gradient-like evolution process.

Proof: Since $\eta(s) \rightarrow p_{0}$ as $s \rightarrow-\infty$, the evolution process $\{S(t, s): t \geqslant s\}$ is asymptotically autonomous at $-\infty$, and thus the truncated process satisfies also item $(d)$ of Theorem 4.4.2, which gives us the result.

With this result, we can look more closely to the behavior of solutions $\xi: \mathbb{R} \rightarrow X$ of the evolution process $\{S(t, s): t \geqslant s\}$ at $-\infty$.

Theorem 4.4.6. Under the hypotheses and notations of Theorem 4.4.5, if $\xi: \mathbb{R} \rightarrow X$ is a bounded global solution for the evolution process $\{S(t, s): t \geqslant s\}$, then there exists $i=1, \cdots, n$ such that $\xi(s) \rightarrow \Gamma_{i, 0}^{*}$ as $s \rightarrow-\infty$.

Proof: Let $\xi: \mathbb{R} \rightarrow X$ be a bounded global solution for the evolution process $\{S(t, s): t \geqslant 0\}$. Then, for each $\tau \in \mathbb{R}$, the function $\xi_{\tau}: \mathbb{R} \rightarrow X$ defined by

$$
\xi_{\tau}(t)=\left\{\begin{array}{ll}
\xi(t), & \text { if } t \leqslant \tau \\
T(t-\tau) \xi(\tau), & \text { if } t \geqslant \tau
\end{array},\right.
$$


is a bounded global solution for the forward truncated evolution process $\left\{S_{\tau}(t, s): t \geqslant s\right\}$. By Theorem 4.4.5, there exists $\tau_{0}<0$ and $i=1, \cdots, n$ such that $\mathrm{d}_{H}\left(\xi_{\tau_{0}}(s), \Xi_{i}^{*}(s)\right) \rightarrow 0$ as $s \rightarrow-\infty$ and since $\mathrm{d}_{H}\left(\Xi_{i}^{*}(s), \Gamma_{i, 0}^{*}\right) \rightarrow 0$ as $s \rightarrow-\infty$ and $\xi_{\tau_{0}}(s)=\xi(s)$ for $s \leqslant \tau_{0}$, the result follows.

Analogously, we can state the result:

Theorem 4.4.7. Let $X$ and $P$ be metric spaces $\varphi: \mathbb{R}^{+} \times P \times X \rightarrow X$ be a cocycle, $\Theta: \mathbb{R}^{+} \times X \rightarrow X$ be the driving system and $\Pi: \mathbb{R}^{+} \times X \times P \rightarrow X \times P$ be the associated skew product semiflow. Assume that $\Pi$ has a global attractor $\mathscr{A},\{\Theta(t): t \geqslant 0\}$ has a global attractor $\mathscr{A}$ and that $p_{0} \in \mathscr{A}$ is a fixed point of $\Theta$. Assume also that there exists a bounded global solution $\eta: \mathbb{R} \rightarrow \mathscr{A}$ such that $\eta(s) \rightarrow p_{0}$ as $s \rightarrow \infty$ and that:

a) There exists $s_{0}>0$ such that $\bigcup_{s \geqslant s_{0}} \mathscr{A}(\eta(s))$ is precompact.

$\left.b^{\prime}\right)$ If $T(t):=\varphi\left(t, p_{0}\right)$, for all $t \geqslant 0$ then $\{T(t): t \geqslant 0\}$ is a generalized gradient-like semigroup with isolated invariant sets $\left\{\Gamma_{1,0}^{*}, \cdots \Gamma_{n, 0}^{*}\right\}$.

$\left.c^{\prime}\right)$ If $S(t, s):=\varphi(t-s, \eta(s))$ for all $t \geqslant s$, the evolution process $\{\varphi(t-s, \eta(s)): t \geq s\}$ possesses $n \in \mathbb{N}$ isolated invariant families

$$
\Psi=\left\{\Xi_{1}(\cdot), \cdots, \Xi_{n}(\cdot)\right\}
$$

which behave upper e lower semi-continuously as $s \rightarrow \infty$, that is

$$
\sup _{1 \leqslant i \leqslant n}\left[d_{H}\left(\Xi_{i}^{*}(s), \Gamma_{i, 0}^{*}\right)+d_{H}\left(\Gamma_{i, 0}^{*}, \Xi_{i}(s)\right)\right] \stackrel{s \rightarrow \infty}{\longrightarrow} 0 .
$$

d') There exist $\mu>0$ such that, if $\xi: \mathbb{R} \rightarrow X$ is a bounded solution of $\{\varphi(t-s, \eta(s)): t \geq s\}$ so that, if there are $t_{0} \in \mathbb{R}$ and $i \in\{1, \cdots, n\}$ with $\sup _{t \geq t_{0}} \operatorname{dist}\left(\xi(t), \Gamma_{i}^{*}\right)<\mu$, then $\lim _{t \rightarrow \infty} \mathbf{d}\left(\xi(t), \Xi_{i}^{*}(t)\right)=$ 0 .

Then, there exists $\tau_{0}>0$ such that for all $\tau \geqslant \tau_{0}$ the backward truncated evolution process $\left\{S_{\tau}(t, s): t \geqslant s\right\}$ is a generalized gradient-like evolution process.

Theorem 4.4.8. Under the hypotheses and notations of Theorem 4.4.7, if $\xi: \mathbb{R} \rightarrow X$ is a bounded global solution for the evolution process $\{S(t, s): t \geqslant s\}$, then there exists $i=1, \cdots, n$ such that $\xi(s) \rightarrow \Gamma_{i, 0}^{*}$ as $s \rightarrow \infty$. 


\subsection{Applications}

Let us now analyze several examples which can be useful for applications.

Example 4.5.1. Consider

$$
\left\{\begin{array}{l}
\dot{x}=r(t, x), \quad t>0, \\
x(0)=x_{0} \in \mathbb{R}^{n},
\end{array}\right.
$$

with $r(t, x)$ from Example 4.1.10, i.e., we have a smooth function $r: \mathbb{R}^{+} \times \mathbb{R}^{n} \rightarrow \mathbb{R}^{n}$ given by

$$
r(t, x)=h(t) f(x)+(1-h(t)) g(x), \text { for all } t \in \mathbb{R}^{+} \text {and } x \in \mathbb{R}^{n},
$$

where $f, g: \mathbb{R}^{n} \rightarrow \mathbb{R}^{n}$ are continuous functions.

We have already shown that $f, g \in H(r)$ and also that there is a connection between $f$ and $g$, and a connection between $g$ and $f$. Thus we cannot have a Morse decomposition in $H(r)$ which separates $f$ and $g$, since each one is connected to the other.

Nevertheless, we can choose one of these global solutions, for example $\xi$, and restrict ourselves to $K \doteq \overline{\xi(\mathbb{R})} \subset H(r)$, which has a non-trivial Morse decomposition, i.e. $M_{1}=\{f\}$ and $M_{2}=\{g\}$, and thus generates a Morse decomposition for the associated skew product semiflow and non-autonomous dynamical system related to (4.5.1).

Example 4.5.2. Consider a function $f: \mathbb{R} \times \mathbb{R}^{n} \rightarrow \mathbb{R}^{n}$ and the non-autonomous dynamical system

$$
\left\{\begin{array}{l}
\dot{x}=f(t, x), \quad t \in \mathbb{R} \\
x(0)=x_{0} .
\end{array}\right.
$$

Assume that there are functions $f_{1}, f_{2}: \mathbb{R}^{n} \rightarrow \mathbb{R}^{n}$ such that

$$
\sup _{x \in \mathbb{R}^{n}}\left\|f(t, x)-f_{2}(x)\right\|_{\mathbb{R}^{n}} \stackrel{t \rightarrow \infty}{\longrightarrow} 0, \quad \text { and } \quad \sup _{x \in \mathbb{R}^{n}}\left\|f(t, x)-f_{1}(x)\right\|_{\mathbb{R}^{n}} \stackrel{t \rightarrow-\infty}{\longrightarrow} 0 .
$$


Now considering the space $C_{b}\left(I \times \mathbb{R}^{n}, \mathbb{R}^{n}\right)$ (see Subsection 4.1.2), we can see that

$$
\left\|\theta(t) f(s, x)-f_{2}(x)\right\|_{k}=\sup _{x \in B_{k}, s \in I_{k}}\left\|f(t+s, x)-f_{2}(x)\right\|_{\mathbb{R}^{n}} \stackrel{t \rightarrow \infty}{\longrightarrow} 0
$$

and

$$
\left\|\theta(t) f(s, x)-f_{1}(x)\right\|_{k}=\sup _{x \in B_{k}, s \in I_{k}}\left\|f(t+s, x)-f_{2}(x)\right\|_{\mathbb{R}^{n}} \stackrel{t \rightarrow-\infty}{\longrightarrow} 0,
$$

for all $k \in \mathbb{N}$, which means that $\theta(t) f \stackrel{t \rightarrow \infty}{\longrightarrow} f_{2}$ and $\theta(t) \stackrel{t \rightarrow-\infty}{\longrightarrow} f_{1}$ in the uniform convergence on bounded sets, thus $H(f)=\{\theta(t) f\}_{t \geqslant 0} \cup\left\{f_{1}, f_{2}\right\}$ and this set has a Morse decomposition $\left\{M_{1}, M_{2}\right\}$ given by

$$
M_{1}=\left\{f_{1}\right\} \quad \text { and } M_{2}=\left\{f_{2}\right\} .
$$

If the skew product semiflow $\{\Pi(t): t \geqslant 0\}$ given by $\Pi(t)\left(x_{0}, g\right)=\left(x\left(t, g, x_{0}\right), g_{t}\right)$ in the phase state $\mathbb{R}^{n} \times H(f)$ has a global attractor $\mathscr{A}$, then $\left\{M_{1}^{\mathscr{A}}, M_{2}^{\mathscr{A}}\right\}$ is a Morse decomposition for $\mathscr{A}$. In this case, if $A_{1}=\left\{x \in \mathbb{R}^{n}:\left(x, f_{1}\right) \in \mathscr{A}\right\}$ and $A_{2}=\left\{x \in \mathbb{R}^{n}:\left(x, f_{2}\right) \in \mathscr{A}\right\}$ then

$$
M_{1}^{\mathscr{A}}=A_{1} \times\left\{f_{1}\right\} \quad \text { and } \quad M_{2}^{\mathscr{A}}=A_{2} \times\left\{f_{2}\right\}
$$

Therefore, the solution $x\left(t, f, x_{0}\right)$ of the problem (4.5.2) converges to $A_{1}$ as $t \rightarrow \infty$ and $A_{2}$ as $t \rightarrow-\infty$. Moreover, we know that $A_{1}\left(A_{2}\right)$ is the global attractor of the problem $\dot{x}=f_{1}(x)$ $\left(\dot{x}=f_{2}(x)\right)$, and if $A_{1}\left(A_{2}\right)$ has a Morse decomposition $\left\{M_{1}^{A_{1}}, \cdots, M_{m}^{A_{1}}\right\}\left(\left\{M_{1}^{A_{2}}, \cdots, M_{p}^{A_{2}}\right\}\right)$ then Theorem 4.4.8 (Theorem 4.4.6) guarantees that there exists $i=1, \cdots, m(j=1, \cdots, p)$ such that $x\left(t, f, x_{0}\right)$ converges to $M_{i}^{A_{1}}$ as $t \rightarrow \infty\left(M_{j}^{A_{2}}\right.$ as $\left.t \rightarrow-\infty\right)$.

Example 4.5.3. Consider the system of autonomous differential equations

$$
\left\{\begin{array}{l}
\dot{v}=f(u, v) \quad t>0 \\
\dot{u}=g(u), \quad t>0 \\
u(0)=u_{0} \in \mathbb{R}^{n}, v(0)=v_{0} \in \mathbb{R}^{n}
\end{array}\right.
$$

where the u component is decoupled, so the system (4.5.3) generates a skew product semiflow. The u-component here may be considered to represent an independent system that drives the 
$v$-component of the system in the sense that

$$
\dot{v}=f(u(t), v)
$$

for any given solution $u(t)$ of $\dot{u}=g(u)$. Assume that the system $\dot{u}=g(u)$ generates a semigroup $\{\Theta(t): t \geqslant 0\}$ in $\mathbb{R}^{n}$, that is, $\Theta(t) u_{0}=u\left(t, u_{0}\right)$, where $u\left(\cdot, u_{0}\right)$ is the unique solution for $t>0$ of the problem

$$
\left\{\begin{array}{l}
\dot{u}=g(u), t>0 \\
u(0)=u_{0} .
\end{array}\right.
$$

Assume also that $\{\Theta(t): t \geqslant\}$ has a global attractor $\mathscr{A}$ with a Morse decomposition $\left\{M_{1}, \cdots, M_{n}\right\}$, that the property of backward uniqueness of $\{\Theta(t): t \geqslant 0\}$ in $\mathscr{A}$ holds and also that the generated skew product semiflow $\{\Pi(t): t \geqslant 0\}$ has a global attractor $\mathscr{A}$. Then we have that for every pair of points $\left(u_{0}, v_{0}\right) \in \mathbb{R}^{n} \times \mathbb{R}^{n}$, the solution $v\left(t, v_{0}, u_{0}\right)$ of the problem

$$
\left\{\begin{array}{l}
\dot{v}(t)=f\left(\Theta(t) u_{0}, v(t)\right), t>0 \\
v(0)=v_{0}
\end{array}\right.
$$

satisfies $v\left(t, v_{0}, u_{0}\right) \stackrel{t \rightarrow \infty}{\longrightarrow} \mathscr{P}_{M_{i_{0}}}\left(M_{i_{0}}^{\mathscr{A}}\right)$, for some $i_{0}=1, \cdots, n$, where $i_{0}$ is such that $\Theta(t) u_{0} \stackrel{t \rightarrow \infty}{\longrightarrow}$ $M_{i_{0}}$

Example 4.5.4. This last example illustrates how we can use the general theory in a more concrete case. Let us consider the planar system

$$
\frac{d}{d t}(x, y)=F(t,(x, y)), \quad t \in \mathbb{R}
$$

Assume that $F(t,(x, y)) \rightarrow F_{1}(x, y)$ as $t \rightarrow-\infty$ and that $F(t,(x, y)) \rightarrow F_{2}(x, y)$ as $t \rightarrow \infty$, where $F_{1}, F_{2}: \mathbb{R}^{2} \rightarrow \mathbb{R}^{2}$ satisfy

1. $F_{1}(x, y)=(f(x), g(x, y))$, where $f(x)=x-x^{3}$ and $g(x, y)=\left(1-x^{2}\right) y-y^{3}$. Clearly they satisfy the conditions of Example 4.5.3; 
2. $F_{2}$ is given in polar coordinates by

$$
F_{2}(r \cos \theta, r \sin \theta)=G(r, \theta)=(-r(r-1)(r-2), 1) .
$$

From the Example 4.5.3, we know the Morse decomposition $\left\{M_{0}^{\mathscr{A}}, M_{1}^{\mathscr{A}}, M_{2}^{\mathscr{A}}\right\}$ for the global attractor $\mathscr{A}$ of the planar system

$$
\left\{\begin{array}{l}
\dot{x}=f(x) \\
\dot{y}=g(x, y)
\end{array},\right.
$$

given a Morse decomposition $\left\{M_{0}, M_{1}, M_{2}\right\}$ for the global attractor $\mathscr{A}=[-1,1] \subset \mathbb{R}$ of the equation $\dot{x}=f(x)$, where $M_{0}=\{-1\}, M_{1}=\{1\}, M_{2}=\{0\}$. Indeed, it is not hard to see that the Morse decomposition is this case is given by $M_{0}^{\mathscr{A}}=\{(-1,0)\}, M_{1}^{\mathscr{A}}=\{(1,0)\}$ and $M_{2}^{\mathscr{A}}=\{(0, y): y \in$ $[-1,1]\}$.

Now, we already know that the system $\frac{d}{d t}(x, y)=F_{2}(x, y), t>0$ generates a generalized gradient-like system, with invariant sets given by $\Xi_{0}=\{0\}, \Xi_{1}=\{(1, \theta): \theta \in[0,2 \pi]\}$ and $\Xi_{2}=$ $\{(2, \theta): \theta \in[0,2 \pi]\}$.

Thus, by Example 4.5.2, we know that every solution $\xi: \mathbb{R} \rightarrow \mathbb{R}^{2}$ of the system (4.5.4) satisfies

(a) $\xi(t) \rightarrow M_{i}^{\mathscr{A}}$ for some $i=1, \cdots, n$ as $t \rightarrow-\infty$,

(b) $\xi(t) \rightarrow \Xi_{j}$ for some $j=0,1,2$ as $t \rightarrow \infty$.

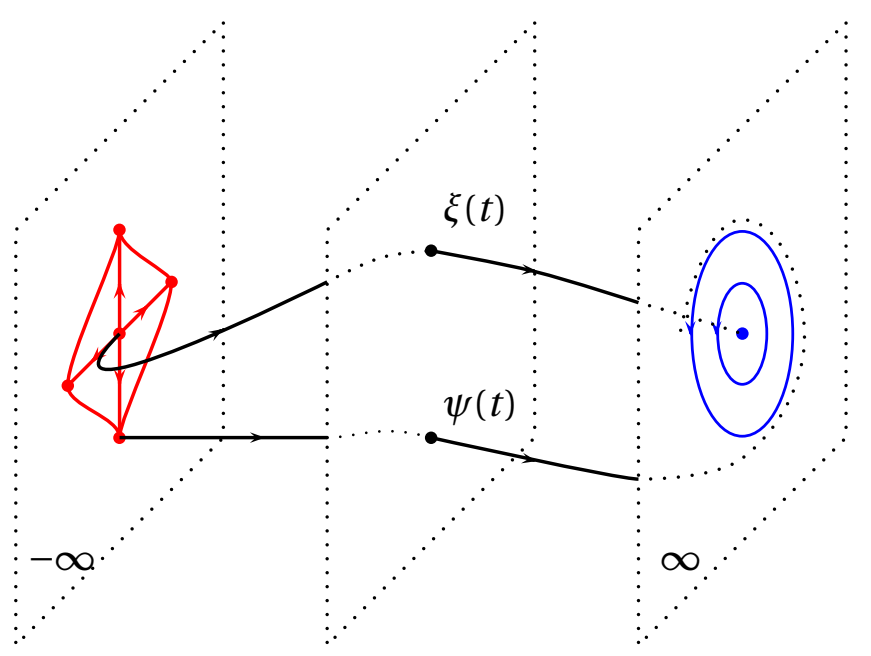

Asymptotic behaviors for solutions $\xi, \psi$ of (4.5.4). 


\section{Bibliography}

[1] E. R. Aragão-Costa, T. Caraballo, A. N. Carvalho and J. A. Langa, Stability of gradient semigroups under perturbations, Nonlinearity 24 2099-2117 (2012).

[2] Babin, A.V., Vishik, M.I., Attractors in Evolutionary Equations Studies in Mathematics and its Applications 25, North-Holland Publishing Co., Amsterdam, 1992.

[3] M. C. Bortolan, T. Caraballo, A. N. Carvalho and J. A. Langa, An estimate on the fractal dimension of attractors of gradient-like dynamical systems, Nonlinear Anal. 75 (2012), no. $14,5702-5722$.

[4] M. C. Bortolan, T. Caraballo, A. N. Carvalho and J. A. Langa, Skew product semiflows and Morse decomposition, submitted for publication.

[5] T. Caraballo, J. C. Jara, J. A. Langa and Z. Liu, Morse decomposition of attractors for non-autonomous dynamical systems, to appear in Advanced Nonlinear Studies.

[6] A. N. Carvalho, Sistemas dinâmicos não-lineares - SMA5880 - Departamento de Matemática ICMC/USP, Class Notes.

[7] A. N. Carvalho, J. A. Langa and J. C. Robinson, Attractors for infinite-dimensional nonautonomous dynamical systems, Applied Mathematical Sciences 182, ISBN 978-1-46144580-7, Springer-Verlag (2012).

[8] A. N. Carvalho, J. A. Langa, J. C. Robinson, A. Suárez, Characterization of nonautonomous attractors of a perturbed infinite-dimensional gradient system. Journal of Differential Equations, 236 (2007) 570-603. 
[9] A. N. Carvalho and J. W. Cholewa, Exponential global attractors for semigroups in metric spaces with applications to differential equations, Ergodic Theory and Dynamical Systems, 31 (6) 1641-1667 (2012).

[10] A. N. Carvalho, J. A. Langa, Non-autonomous perturbation of autonomous semilinear differential equations: Continuity of local stable and unstable manifolds, J. Differential Equations 233 622-653 (2007).

[11] A. N. Carvalho, J. A. Langa, An extension of the concept of gradient systems which is stable under perturbation, J. Differential Equations, 246 (7) 2646-2668 (2009).

[12] A. N. Carvalho, J. A. Langa and J. C. Robinson, Attractors for infinite-dimensional nonautonomous dynamical systems, Applied Mathematical Sciences 182, Springer-Verlag, New York 2012.

[13] A. N. Carvalho, J. A. Langa, J. C. Robinson, Lower semicontinuity of attractors for nongradient dynamical systems, Ergodic Theory and Dynamical Systems, 29 (6) 1765-1780 (2009).

[14] D. Cheban, P. E. Kloeden and B. Schmalfuß, The relationship between pullback, forwards and global attractors of nonautonomous dynamical systems, Nonlinear Dyn. Syst. Theory 2 125-144 (2002)

[15] V. V. Chepyzhov, and M. I. Vishik, Attractors for Equations of Mathematical Physics, Colloquium Publications, vol.49. American Mathematical Society, Providence, RI (2001)

[16] C. Conley, Isolated invariant sets and the Morse index. CBMS Regional Conference Series in Mathematics, 38. American Mathematical Society, Providence, R.I. (1978).

[17] Hale, J.K., Asymptotic Behavior of Dissipative Systems, Mathematical Surveys and Monographs Number 25 (American Mathematical Society, Providence, RI) (1988).

[18] J. K. Hale, L. Magalhães and W. M. Oliva Dynamics in Infinite Dimensions, Applice Mathematical Sciences 47, Second Edition, Springer Verlag (2002).

[19] D. Henry, Geometric Theory of Semilinear Parabolic Equations, Lecture Notes in Mathematics 840, Springer-Verlag, Berlin, (1981).

[20] Hunt, Brian R.; Kaloshin, Vadim Yu. Regularity of embeddings of infinite-dimensional fractal sets into finite-dimensional spaces. Nonlinearity 12 (1999), no. 5, 1263-1275. 
[21] M. Hurley, Chain recurrence, semiflows and gradients, J. Dyn. Diff. Equations 7 437-456 (1995).

[22] Kahane, J.P., Measures et dimensions, Turbulence and the Navier Stokes equation, Lecture Notes in Mathematics 565 Springer-Verlag, New York, (1976).

[23] I.N. Kostin, Rate of attraction to a non-hyperbolic attractor, Asymptot. Anal. 16 (1998), no. 3-4, $203 Đ 222$.

[24] P. Kloeden and M. Rasmussen, Nonautonomous dynamical systems, Mathematical Surveys and Monographs 176, AMS, Providence RI 2011.

[25] Ladyzhenskaya, O.A., Attractors for semigroups and evolution equations, Leizioni Lincee, Cambridge Univ. Press, Campridge, UK (1991)

[26] J.A. Langa, J.C. Robinson \& A. Suárez, Stability, instability, and bifurcation phenomena in non-autonomous differential equations, Nonlinearity 15 (2002), no. 3, 887-903.

[27] J.A. Langa, J.C. Robinson, A. Rodríguez-Bernal and A. Suárez, Permanence and asymptotically stable complete trajectories for non-autonomous Lotka-Volterra models with diffusion, SIAM J. Math. Anal. 40 (6) (2009), 2179-2216.

[28] J.A. Langa, A. Rodríguez-Bernal, A. Suárez, On the long time behavior of nonautonomous Lotka-Volterra models with diffusion via the sub-supertrajectory method, J. Differential Equations, 249 (2010), 414-445.

[29] J. A. Langa and A. Suárez, Pullback permanence for non-autonomous partial differential equations, Electron. J. Differential Equations (2002), No. 72, 20 pp.

[30] J. Mallet-Paret, Negatively invariant sets of compact maps and an extension of a theorem of Cartwright, J. Differential Equations, 22 331-348 (1976).

[31] R. Mañé, On the dimension of the compact invariant sets of certain non-linear maps, Lecture Notes in Mathematics 898, Springer-Verlag, New York, pp. 230-242, 1981.

[32] K. Mischaikow, H. Smith and H. R. Thieme, Asymptotically autonomous semiflows: chain recurrent and Lyapunov functions, Trans. Amer. Math. Soc. 347 (5) 1669-1685 (1995).

[33] J. R. Munkres, Topology, Second Edition, Pearson Education (2000). 
[34] D.E. Norton, The fundamental theorem of dynamical systems, Comment. Math., Univ. Carolinae 36 (3) 585-597 (1995).

[35] M. Patrão, Morse decomposition of semiflows on topological spaces, J. Dyn. Diff. Equations 19 (1) (2007), 181-198.

[36] M. Patrão and Luiz A.B. San Martin, Semiflows on topological spaces: chain transitivity and semigroups, J. Dyn. Diff. Equations 19 (1) (2007), 155-180.

[37] C. Pötzsche, Geometric Theory of Discrete Nonautonomous Dynamical Systems, Lecture Notes in Mathemathics 2002, Springer-Verlag, New York 2010.

[38] M. Rasmussen, Attractivity and Bifurcation for Nonautonomous Dynamical Systems, Lecture Notes in Mathemathics 1907, Springer-Verlag, New York 2007.

[39] M. Rasmussen, Morse decomopsitions of nonautonomous dynamical systems, Trans. Amer. Math. Soc. 359 (10) 5091-5115 (2007).

[40] G. Raugel, Global Attractors in Partial Differential Equations, in Handbook of Dynamical Systems, volume 2, B. Fiedler editor, (2002), Elsevier Sciences, B. V., pp 885-982.

[41] J.C. Robinson, Dimensions, Embeddings, and Attractors, Cambridge Tracts in Mathematics 186 (2010).

[42] Robinson, J.C. Infinite-Dimensional Dynamical Systems, From Basic Facts to Actual Calculations, Cambridge University Pres, Cabridge UK (2001).

[43] A. Rodríguez-Bernal and A. Vidal-López, Existence, uniqueness and attractivity properties of positive complete trajectories for non-autonomous reaction-diffusion problems Discrete Contin. Dyn. Syst. 18 (2-3) 537-567

[44] K. P. Rybakowski, The homotopy index and partial differential equations, Universitext, Springer-Verlag (1987).

[45] G. R. Sell and Y. You, Dynamics of evolutionary equations. Applied Mathematical Sciences, 143. Springer-Verlag, New York, 2002.

[46] Temam, R. Infinite dimensional dynamical systems in mechanics and physics. New York: Springer. 


\section{Index}

absorption, 8

pullback, 32

attraction, 8

pullback, 32

attractor

pullback, 33

attractor-repeller pair, 19

bounded dissipative

pullback, 32

chain recurrent, 13

compact dissipative, 9

disjoint family of isolated invariants, 13

$\epsilon$-chain, 13

equilibrium point, 7,12

evolution process, 31

generalized gradient-like, 36

linear,31

exponentially attracting,26

family of semigroups

collective asymptotically compact at $\eta=0,17$

continuous, 10

fixed point, 7

gradient semigroup, 12

Hausdorff semidistance, 8 homoclinic structure,16

for a process, 36

image, 6, 31

invariance, 8,32

positive, 8

local attractor,19

local stable set,14

local unstable set,11,14

Lyapunov function, 24

Morse decomposition, 21

$\omega$-limit

pullback, 34

$\omega$-limit set, 7

orbit,632

global, 7

partial, 6

positive, 6

pullback, 32

repeller,19

semicontinuity

lower,10

upper,10

semigroup, [5]

asymptotically compact, 9 
bounded, 6

bounded dissipative, 9

eventually bounded,6

generalized gradient-like, 13

gradient like relative to,13

gradient relative to $\Psi, 24$

point dissipative, 9

solution, 6

bounded backwards,33

global,7

stationary, 7

stable set, 14

unstable set,11, 14, 36 